\title{
Run-09 pC Polarimeter Analysis
}

I. Alekseev, E. Aschenauer, G. Atoyan, A. Bazilevsky, R. Gill, H. Huang, S. Lee, X. Li Y. Makdisi, B. Morozov, I. Nakagawa, D. Svirida and A. Zelenski

Brookhaven National Laboratory, Upton, NY 11973, USA

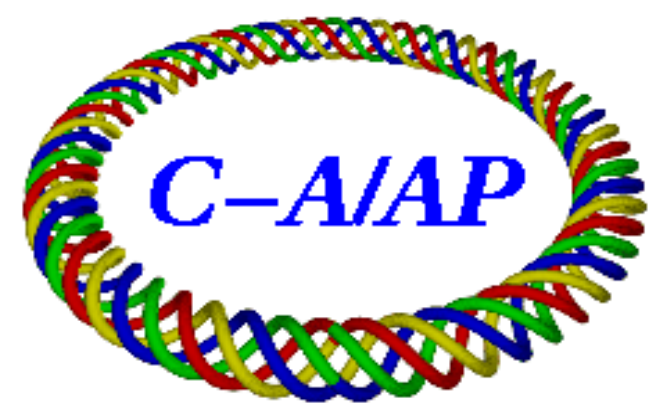

\section{Collider-Accelerator Department Brookhaven National Laboratory Upton, NY 11973}

Notice: This document has been authorized by employees of Brookhaven Science Associates, LLC under Contract No. DE-AC02-98CH10886 with the U.S. Department of Energy. The United States Government retains a nonexclusive, paid-up, irrevocable, world-wide license to publish or reproduce the published form of this document, or allow others to do so, for United States Government purposes. 


\section{Run-09 pC Polarimeter Analysis}

I. Alekseev, E. Aschenauer, G. Atoyan, A. Bazilevsky, R. Gill, H. Huang, S. Lee, X. Li, Y. Makdisi, B. Morozov, I. Nakagawa, D. Svirida and A. Zelenski

Analysis of PC polarimeter data at $\sqrt{s}=200$ and $500 \mathrm{GeV}$ from Run9 is presented. Final polarization results, fill-by-fill, for blue and yellow beams, as to be used by RHIC experiments (in collisions) are released and collected in

http://www4.rcf.bnl.gov/ cnipol/pubdocs/Run090ffline/. Global relative systematic uncertainties $\delta P / P$ (to be considered as correlated from fill to fill) are $4.7 \%$ for $100 \mathrm{GeV}$ beams, and $8.3 \%$ (12.1\%) for blue (yellow) $250 \mathrm{GeV}$ beams. For a product of two beam polarizations $P_{B} \cdot P_{Y}$ (used in double spin asymmetry measurements) the relative uncertainty $\delta\left(P_{B} \cdot P_{Y}\right) /\left(P_{B} \cdot P_{Y}\right)$ is $8.8 \%$ for $100 \mathrm{GeV}$ beams and $18.5 \%$ for 250 $\mathrm{GeV}$ beams. For the average between two beam polarization $\left(P_{B}+P_{Y}\right) / 2$ (used in single spin asymmetry measurements, when data from two polarized beams are combined) the relative uncertainty is $4.4 \%$ for $100 \mathrm{GeV}$ beams and $9.2 \%$ for $250 \mathrm{GeV}$ beams. Larger uncertainties for $250 \mathrm{GeV}$ beams relate to significant rate related systematic effects experienced in the first part of Run9 (due to thicker targets used and smaller trans. beam size at higher beam energy). 


\section{Contents}

1 Introduction $\quad 3$

2 Response to alphas, energy calibration $\quad 4$

$\begin{array}{lll}3 & \text { Response to recoil carbons } & 7\end{array}$

4 Energy and Time of Flight correction $\quad 12$

5 Data Quality Assurance $\quad 21$

5.1 Strip Quality Assurance . . . . . . . . . . . . . . . . . 21

5.2 Bunch Quality Assurance . . . . . . . . . . . . . . . . 22

6 Polarization measurements 23

6.1 Polarimeters-1 vs Polarimeters-2 . . . . . . . . . . . . . . . . . 23

6.2 Polarization profile . . . . . . . . . . . . . . . . 23

6.3 Normalization to HJet . . . . . . . . . . . . . . . . . . . 30

6.4 Polarization decay in a fill . . . . . . . . . . . . . . . . . . . 31

6.5 Spin direction in $\mathrm{pC}$ (up-down vs left-right asymmetries) . . . . . . . . . 36

6.6 Final polarizations and uncertainties . . . . . . . . . . . . 36

$\begin{array}{lll}7 & \text { Summary } & 43\end{array}$

A Systematic effects studies $\quad 43$

A.1 Rate issues . . . . . . . . . . . . . . . . . . . 43

A.2 Hamamatsu detectors vs BNL detectors . . . . . . . . . . . 45 


\section{Introduction}

In this analysis we discuss the analysis of RHIC run 2009 pC data (February - July 2009). The pC measurements were performed for $250 \mathrm{GeV}$ beams (February - April) and for $100 \mathrm{GeV}$ beams (April - July). Analysis apporach is basically very similar to one developed for 2005 pC data analysis [1] and also used for 2006 and 2008 data analysis [2], and includes the following steps:

- Energy calibration with alpha source

- Determination of energy and time corrections, for each measurement (run)

- QA analysis to mask bad strips, for each measurement (run)

- QA analysis to reject bad measurements (runs)

- Polarization profile analysis

- Normalization of pC measurements to HJet absolute polarization measurements.

- Evaluation of systematic uncertainties for polarization measurements

Before 2009 RHIC Run the pC polarimeter system was upgraded, so that we had two polarimeters in each ring, with their own target and detector systems, and which shared the same DAQ system (shapers, WFDs). It allowed us not only to perform measurements of both vertical and horizontal polarization profiles every time the pC polarization measurement is done during RHIC store, and test new detectors, but also to provide a very important cross check for our results by comparing the measurements obtained by the two polarimeters, in each ring. In the following we call polarimeters Blue1 and Blue2 (Blue2 is upstream) in the blue ring and Yellow1 and Yellow2 (Yellow1 is upstream) in the yellow ring.

In each polarimeter, carbon targets were installed on two target ladders, one with six horizontal targets and another one with six vertical targets. Two identical target mortion mechanisms were used to perform measurements either with vertical or horizontal targets.

The detector system in each polarimeter to detect recoil carbon consisted of 6 Silicon strip detectors (numbered from 0 to 5 in the following) mounted in a vacuum chamber at azimuthal angles of 90 (detectors 1 and 4 ) and \pm 45 degrees (detectors $0,2,3$ and 5) relative to vertical direction (the stable proton spin direction at RHIC), as shown in Fig. 1 Each of the detectors was segemented into 12 srtrips (so 72 strips numbered from 0 to 71 in the following). All detectors except the 90 degree detectors in Blue2 and Yellow2 were identical ones manufactured by the BNL's Instrumentation Division. The Hamamatsu strip photodiods (also segmented into 12 strips ) were instaled in 90 degree detector slots in Blue2. The Hamamatsu single photodiods (two pairs) were installed in the 90 degree slots in Yellow2, which had their own (slow) DAQ system and are not used in the current analysis.

In Run9 we experienced serious systematic problems in $\mathrm{pC}$ measurements related to high event rates (a factor 2-3 higher than in previous years), which mainly come from the

smaller transverse beam size (higher luminosity) for $250 \mathrm{GeV}$ beams and on the average 


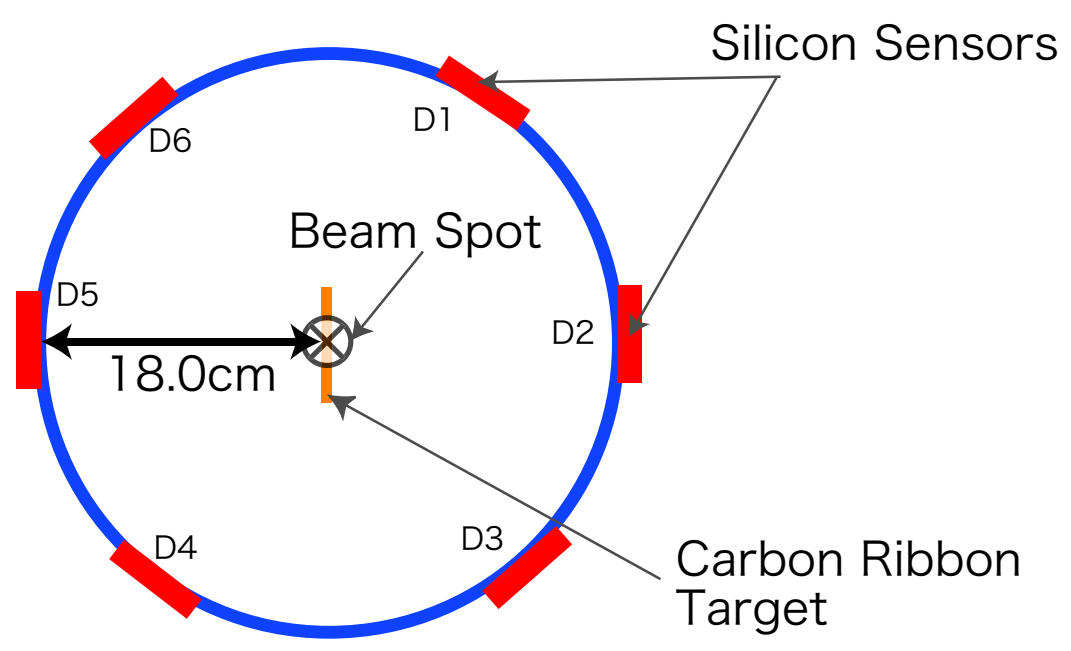

Figure 1. A cross section of the RHIC pC Polarimeter setup. Notice the detector numbering in the text is from 0 to 5 (which corresponds to D1 to D6 in this Fig.)

thicker targets used for the measurements. It will be discussed in more details below in this note.

Since 2008 the main pC operational mode is "target scan": during this measurement the target was moving across the beam with uniform spead. This allowed us to measure not only the average polarization across the beam, but also the polarization and intensity profiles (beam emittance). Sometimes measurements were done in "fixed target" mode: the target was placed at beam maximum intenity. In this analysis we used only the scan measurements.

pC polarization measurements in Run9 physics fills were organized in such a way that if for example Polarimeter-1 was used for a horizontal scan (with vertical target), Polarimeter-2 was used for a vertical scan (with horizontal target). If a measurement is done with a horizontal target the 90 degree detectors (detectors 1 and 4) are partially shadowed, therefore they were not included in the polarization analysis.

Fig. 2 and 3 summarize the information on targets used in the Run9 measurements.

In all plots in this note only statistical uncertainties are shown unless mentioned otherwise.

\section{Response to alphas, energy calibration}

The energy calibration of detectors is performed using a ${ }^{241} \mathrm{Am} \alpha$ source. Its energy spectrum contains two major lines $5.486 \mathrm{MeV}$ (85\%) and $5.443 \mathrm{MeV}$ (13\%), which can not be resolved in our measurements. Since the kinetic energy of $\alpha$ is more than a factor of 5 higher than the maximal carbon energy used for the regular polarization measurements, the signal output for the alpha calibration runs was attenuated by a factor of 5 .

Dedicated calibration runs were performed several times throughout Run9 to monitor gain drifts and operability of strips. Fig. 4 shows a typical result from a calibration run. The alpha peaks show up at about 180 counts in all strips. Two edge strips in detector 1 and 6 (strips 0,1 and 70,71) were shadowed so were not well exposed to alphas. Their calibration coefficient was assigned from the average over 10 good strips 


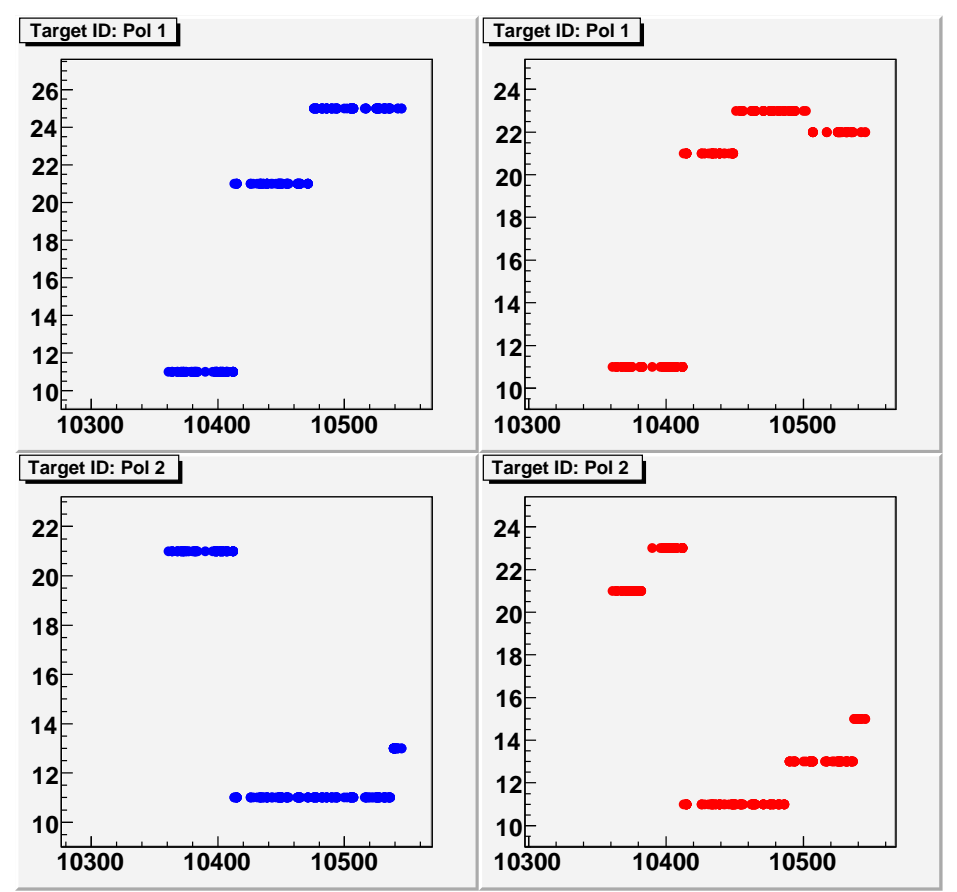

Figure 2. Target ID in $\sqrt{s}=500 \mathrm{GeV}$ measurements vs fill number (each fill usually had several measurements, all of them are shown here); top left - Blue1, bottom left - Blue2, top right - Yellow1 and bottom right - Yellow2; Target ID is 10+id for vertical targets and $20+\mathrm{id}$ for horizontal targets, where id is the target identificator on the target ladder (vertical or horizontal).
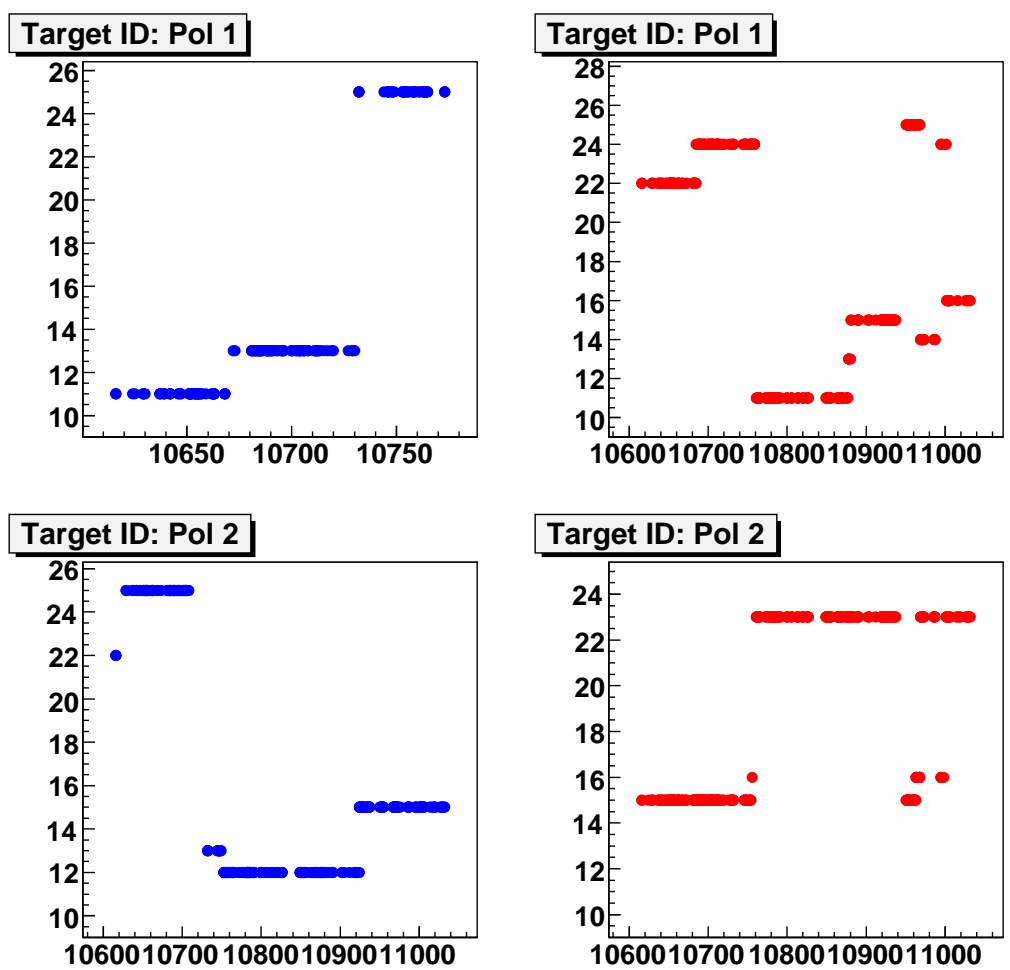

Figure 3. The same as Fig. 2, but for $\sqrt{s}=200 \mathrm{GeV}$ measurements; notice that Blue1 didn't have measurements for fills $>10773$. 


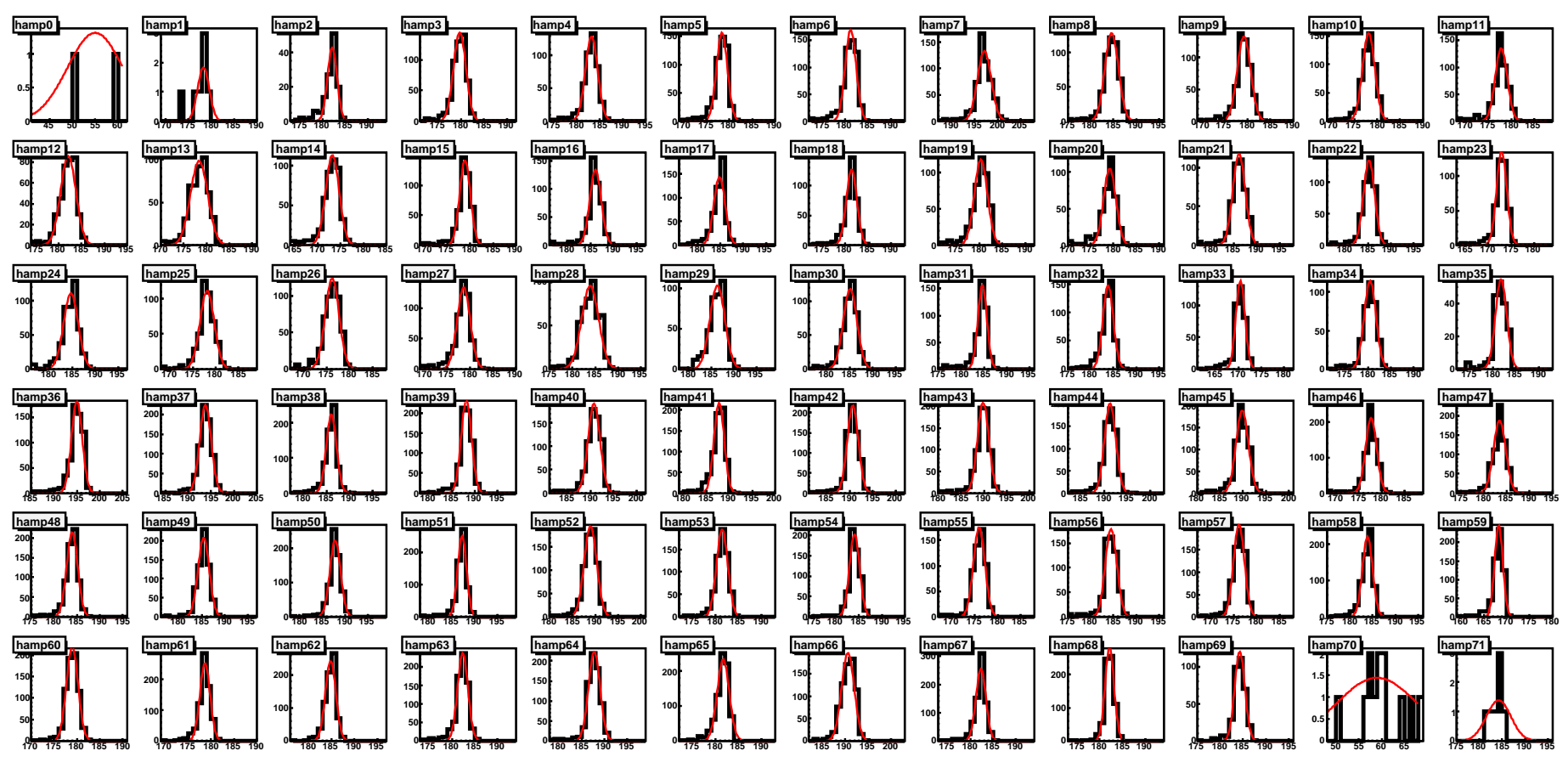

Figure 4. Amplitude response to alphas for the April 29 calibration run for Blue1, for each of the 72 strips; red lines represent the gaussian fits.

in the corresponding detector. Slightly lower response (at $\sim 150$ counts) was observed for the Hamamatsu strip photodiods at 90 degree in Blue2.

The strip by strip gain drift in each polarimeter was monitored by the comparison of the alpha peak position measured in different calibration runs. Fig. 5 shows a typical example of the strip-by-strip comparison of alpha peaks measured by the Blue1 detectors on April 29 and July 6. This particular example shows an average shift of about $0.3 \%$. Only strip 33 (in detector 2) showed an abnormal behavior in Blue1; it was excluded from the analysis.

Fig. 6 and 7 show a summary of the position variation of the alpha peak in all calibration runs in Run9. The red points and error bars represent Mean and RMS values, and blue points and error bars represent mean and sigma from the gaussian fit of the strip-by-strip relative shift, see for example Fig. 5. The points in which red error bars are considerably larger than blue error bars corresponds to the measurements in which one or more strips showed abnormal shifts. These are strip 33 in Blue1 and Blue2 (they correspond to the same WFD channel), and strips 50, 53 and 55 in Yellow1. They were excluded from the offline polarization analysis.

The different detectors in one polarimeter behaved in a similar way, an example is shown in Fig. 8.

As seen from the figures the maximal variation of the average amplitude for $\alpha$ particles didn't exceed $2 \%$ throughout Run9, which if not corrected would lead to a maximum shift of $2 \%$ in measured asymmetries. Actually our analysis technique for energy and time correction (from "Dead layer" and T0 fit), to be discussed below, corrects partially (by about a half) for the gain shift (attributing the gain shift to a change in the effective "Dead 


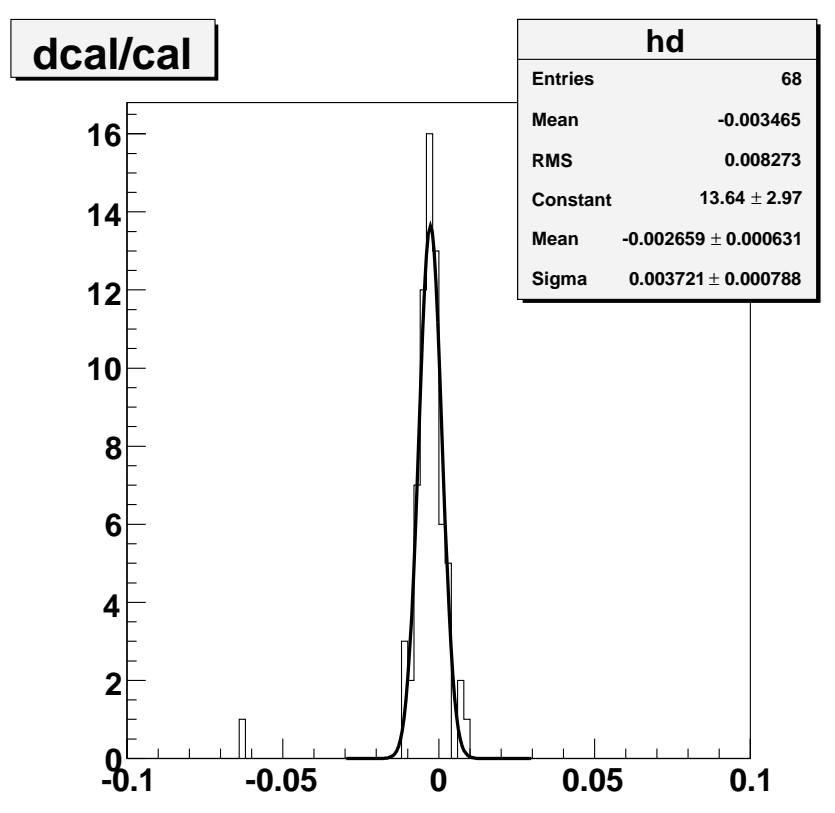

Figure 5. Relative shift in the alpha peak positions measured for the Blue1 strips on July 6 vs April 29. (The outlier on the plot corresponds to strip 33.)

layer"), such the residual effect on the polarization measurements will be at maximum $1 \%$ for a $2 \%$ gain shift.

From these quantitative considerations we can safely fix the energy calibration coefficients (gains) for Run9.

\section{Response to recoil carbons}

Fig. 9 shows the typical response of the pC polarimeter to recoil carbon ions - a bananalike band in time of flight vs energy distribution measured by the Si detectors. Two corrections are necessary: the time of flight offset $(t 0)$ and the correction for the energy loss in the Si dead layer, this corrects carbon deposited energy to kinetic energy. As in previous years analyses, these corrections were obtained from the kinematical fit to the banana extracting two parameters, $t 0$ and the effective dead layer $x_{D L}$ :

$E_{k i n}=E_{\text {meas }}+E_{\text {loss }}\left(x_{D L}, E\right)=\frac{1}{2} \cdot M \cdot \frac{L^{2}}{\left(t_{\text {meas }}+t_{0}\right)^{2}}$

where $M$ is the carbon mass, $L$ is the flight path length (distance between the target and detector, $\sim 18 \mathrm{~cm}$ ), $E_{\text {meas }}$ and $t_{\text {meas }}$ are measured (depositted) energy and time of flight.

This effective dead layer includes not only the real dead layer but also other effects, which lead to the distortion of energy measurements in the polarimeter system. In the past, if measurements were performed with newly installed detectors at good conditions 

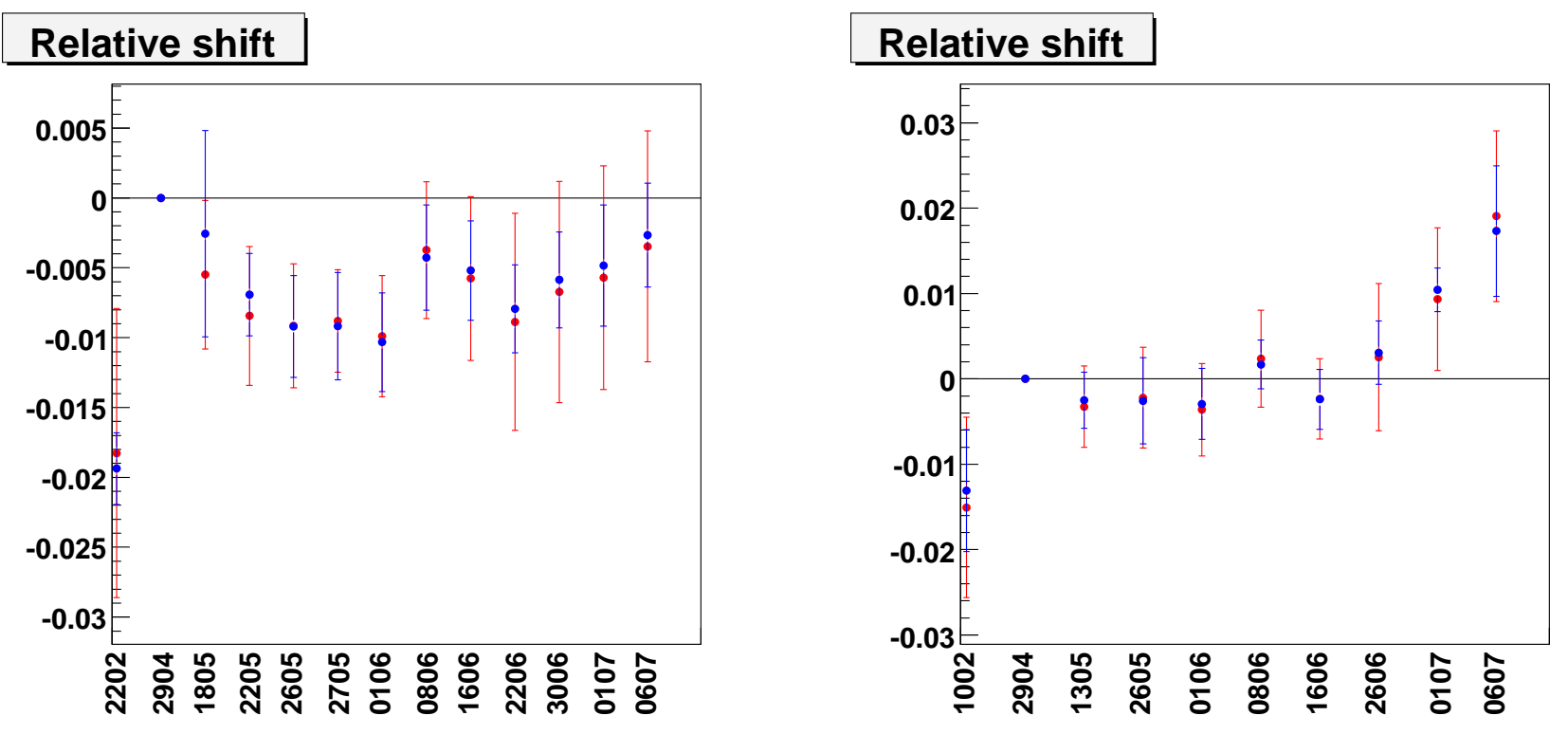

Figure 6. The average over the polarimeter relative variation of the alpha peak position, left - for Blue1, right - for Blue2, for alpha calibration runs in Run9; results are shown from Feb 22 ("2202" in Blue1) and Feb 10 ("1002" in Blue2) to July 6 ("0607"); the relative comparison is performed to results from April 29 ("2904"). The red points and error bars represent Mean and RMS values, and blue points and error bars represent mean and sigma from the gaussian fit of the distribution of the strip-to-dstrip relative shift, i.e Fig. 5.

\section{Relative shift}

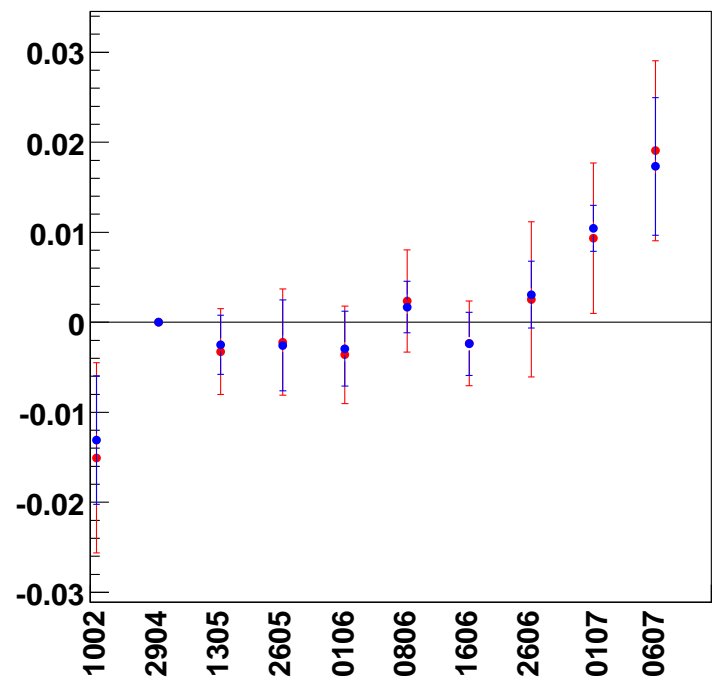

\section{Relative shift}

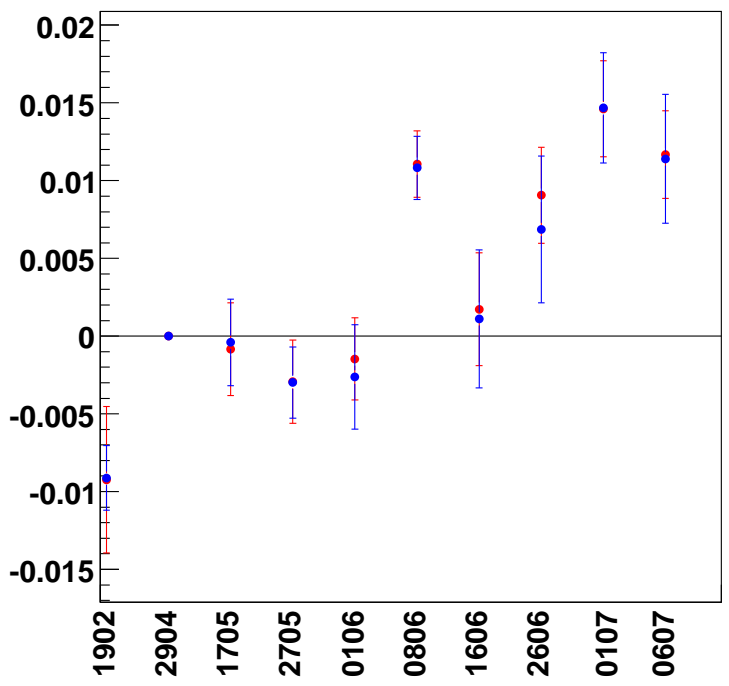

Figure 7. The same as in Fig. 6, but for Yellow1 (left) and Yellow2 (right). 

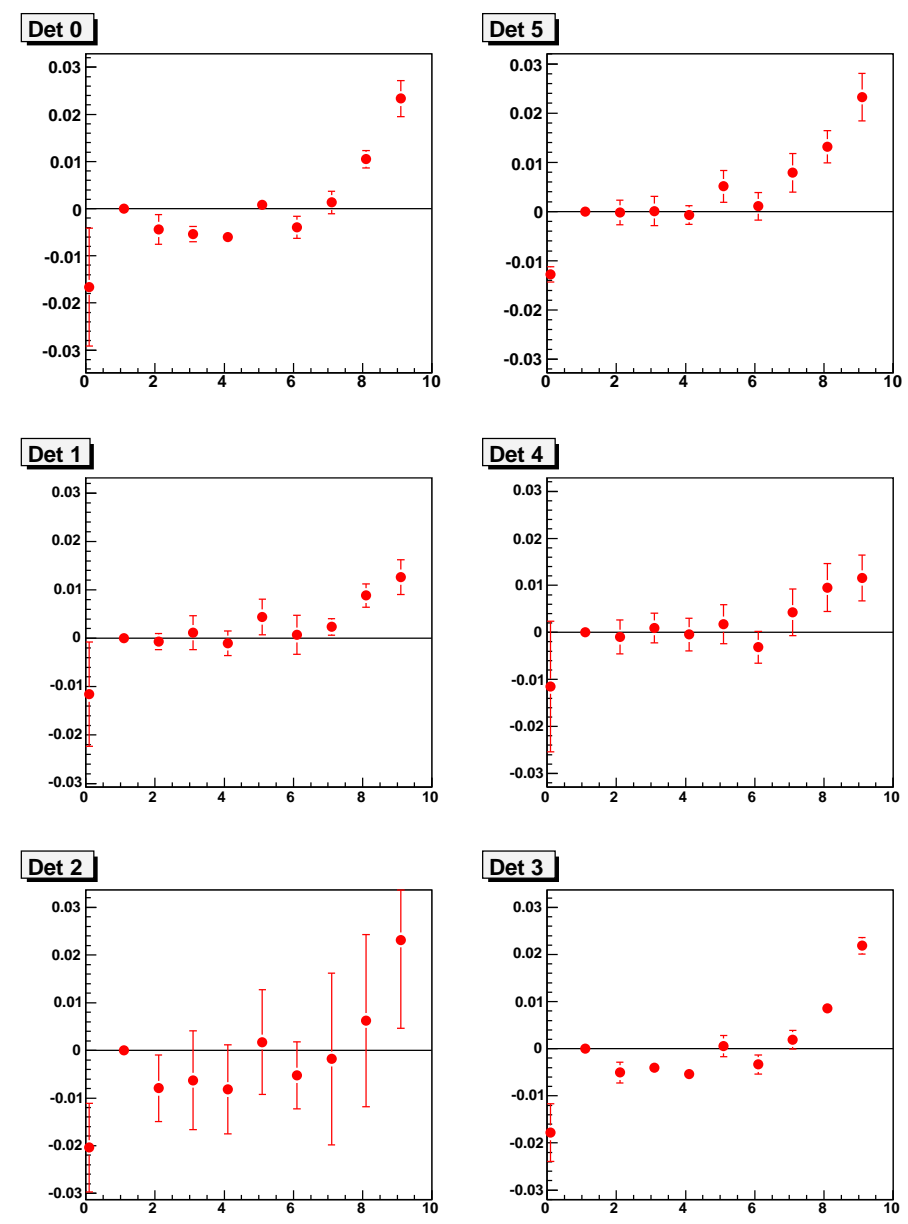

Figure 8. Detector average relative variation of alpha peak position in Blue2; 6 detectors are shown; points and error bars represent Mean and RMS values of the distribution of the strip-to-strip relative shift, i.e. Fig. 5. 


\section{ToF (ns) vs Edep (keV)}

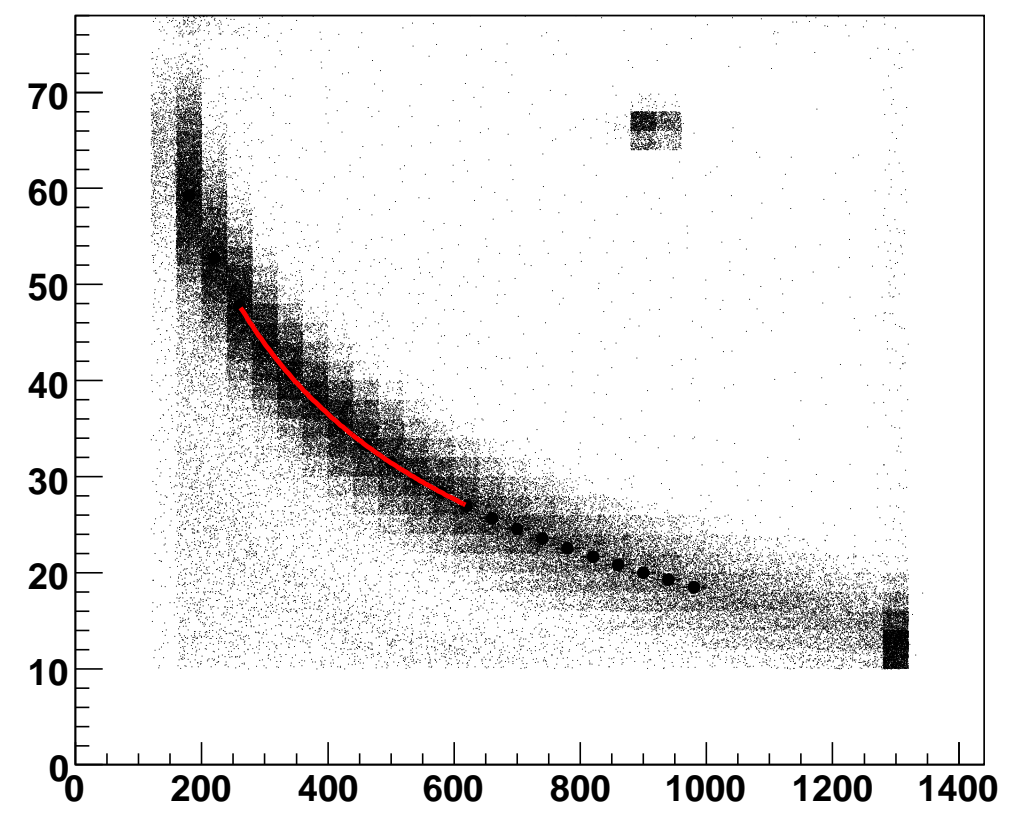

Figure 9. Example of the pC polarimeter response to Carbon ions ("banana") from run 10765.006 (Blue1), strip 1: ToF vs depositted energy. Red line is a kinematical fit with two free parameters, $t 0$ and $x_{D L}$; the extracted parameter values for this case are $t 0=-21$ ns and $x_{D L}=63 \mu \mathrm{g} / \mathrm{cm}^{2}$; the fitting range in depositted energy roughly corresponds to 400-900 keV range in kinetic energy (after correction for the deposited energy due to $x_{D L}$ ) - this is the range used for the asymmetry (beam polarization) measurements in pC. Activity near $E_{d e p} \sim 1000 \mathrm{keV}$ and ToF 65 ns is the system response from generator pulses used for monitoring (discussed in Section A.1.)

(low event rate, thin uniform target etc.), the extracted effective dead layer was usually in reasonable agreement with the real dead layer of $\mathrm{Si}$ detectors calculated from the $\mathrm{p}+$ doping layer depth $[1,3]$.

The stability of the energy and time of flight measurements in a polarimeter can be monitored by fill or run dependence of the reconstructed $\mathrm{C}$ mass from the measured time of flight $t$ (corrected for $t_{0}$ ) and kinetic energy $E_{k i n}$. Fig. 10 and 11 show the reconstructed mass in the 2009 pC measurements. The energy and time of flight corrections (parameters $x_{D L}$ and $t 0$ are fixed for all measurements in each polarimeter strip-by-strip at values obtained at the beginning of Run9 (from runs 10328.002, 10346.204, 10328.102 and 10346.306 for Blue1, Blue2, Yellow1 and Yellow2, respectively). These parameters have been used for the online values throughout Run9.

The variation in the reconstructed mass is correlated with event rate, which is defined as the number of carbon events in the banana plot in the $E_{k i n}$ range $400-900 \mathrm{keV}$. The history of the event rate per strip in Run9 pC measurements is shown on Fig. 12 and 13. For comparison, the Run6 (Run8) rates were below $30 \mathrm{kHz}(60 \mathrm{kHz})$ per strip. As it 


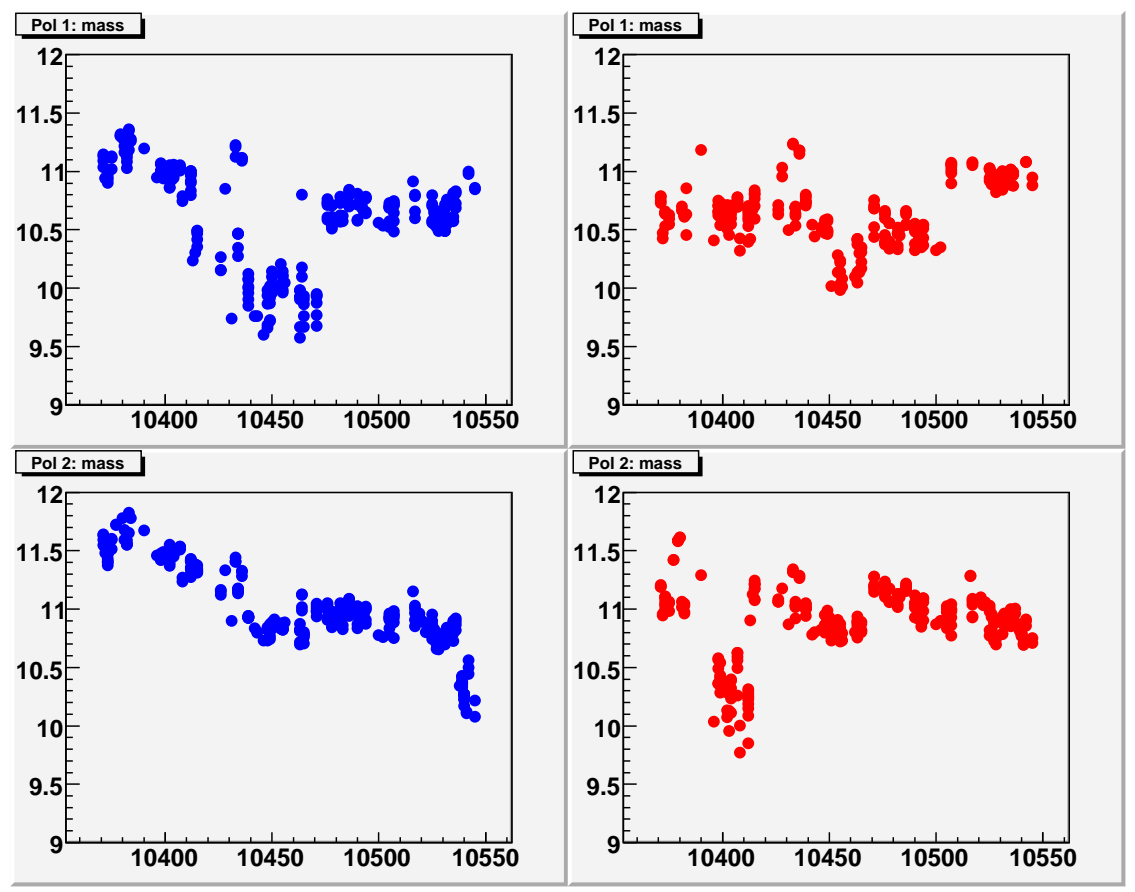

Figure 10. Reconstructed $\mathrm{C}$ mass $\left(\mathrm{GeV} / \mathrm{c}^{2}\right)$ in $\sqrt{s}=500 \mathrm{GeV}$ measurements vs fill number (each fill usually had several measurements, all of them are shown here); top left - Blue1, bottom left - Blue2, top right - Yellow1 and bottom right - Yellow2.
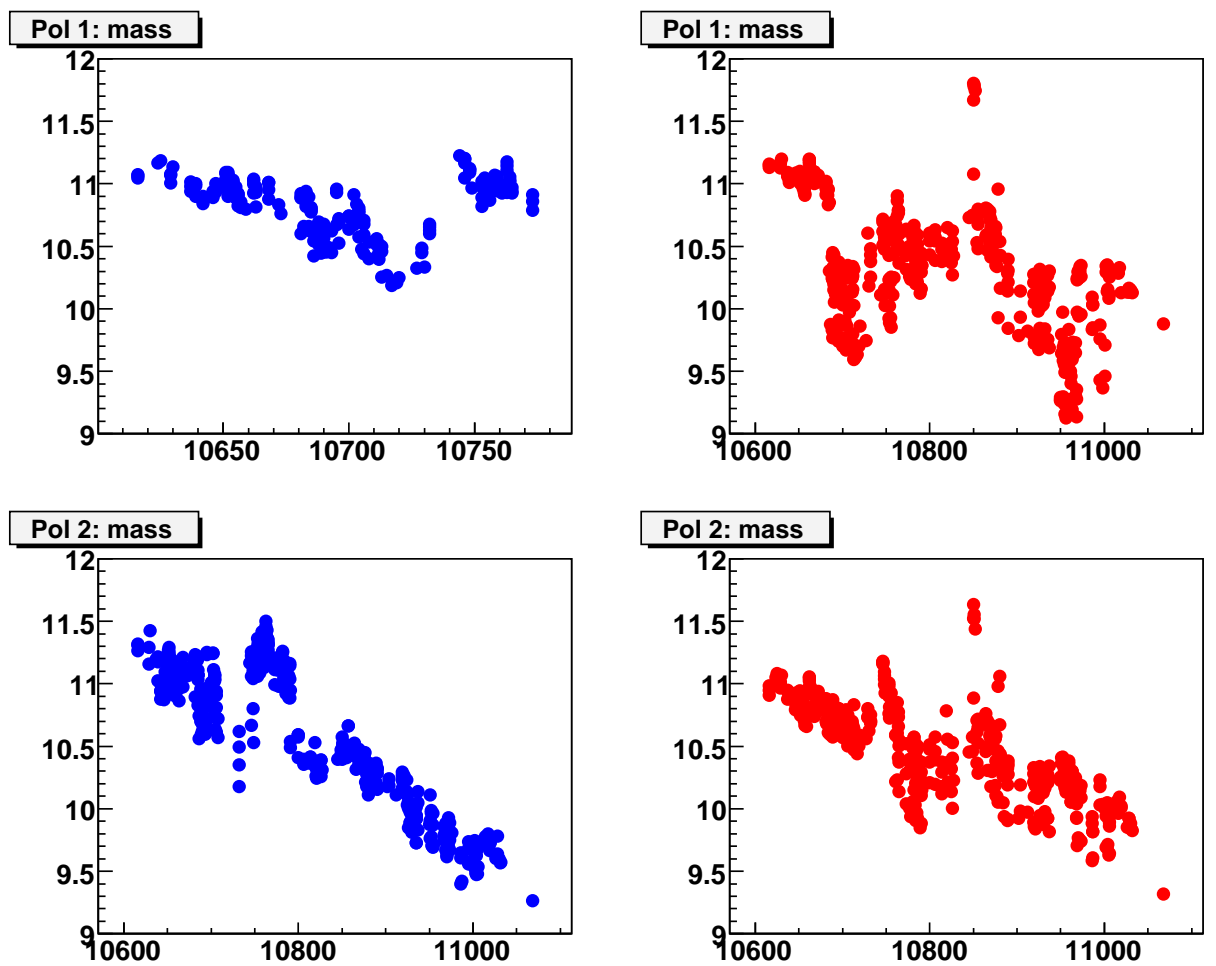

Figure 11. The same as Fig. 10, but for $\sqrt{s}=200 \mathrm{GeV}$ measurements; notice that Blue1 didn't have measurements for fills $>10773$. 
is seen from Fig. 14 and 15 the correlation between reconstructed $\mathrm{C}$ mass and rate has several components, meaning that event rate is not the only observable, which affects the reconstructed mass. The mass is calculated from two measured values, the time of flight and the energy. They will be discussed in the next section.

\section{Energy and Time of Flight correction}

Similar to previous years analyses $[1,2]$ we performed banana fit to extract the parameters $t 0$ and $x_{D L}$ as in Fig. 9 for all pC measurements. As it is seen from Fig. 16 and 17, t0 globally drifted to smaller values, but to a different extent: e.g. by less than $1 \mathrm{~ns}$ in Yellow1 for $\sqrt{s}=500 \mathrm{GeV}$ data, and $\sim 5$ ns in Blue2 for $\sqrt{s}=200 \mathrm{GeV}$ data. A similar trend was observed in previous year Spin Runs. The nature of this effect is not yet well understood. Fig. 18 shows that the global trend for the $t 0$ is about the same in all detectors (here in Blue2). Notice that in this Fig. detectors 1 and 4 are the Hamamatsu strip photodiods, all others are BNL's Si detectors. The global effect is approximately not detector dependent.

It's worth commenting that the $t 0$ drift can not be just an artifact of the fit. If it would be so, it had to be compensated by a shift in the energy measurements $\left(x_{D L}\right)$ according to Eq. (1). For example, for the Blue2 polarimeter, the shift in $t 0$ by 5 ns from the beginning to the end of $\sqrt{s}=200 \mathrm{GeV}$ part of Run9 would correpond to a shift in $x_{D L}$ by $\sim 30 \mu \mathrm{g} / \mathrm{cm}^{2}$ or a $\sim 20 \%$ shift in the analysing power (or measured beam polarization). This is not supported by the comparison of Blue2 and the HJet measurements (shown later in this note).

The history of $x_{D L}$ is shown in Fig. 19 and 20. Fig. 21 shows that the $x_{D L}$ global trend is about the same in all detectors (here in Blue2). Notice that on this Fig. detectors 1 and 4 are the Hamamatsu strip photodiods, all others are BNL's Si detectors. So the global effect is not detector dependent.

The extracted parameter $x_{D L}$ shows a clear correlation with the event rate (Fig. 22 and 23), which indicates that $\mathrm{pC}$ polarimeter energy measurements are affected by high event rates. At the same time $t 0$ doesn't show a clear correlation with rates (Fig. 24 and 25). So using the parameters $x_{D L}$ and $t 0$ we decoupled the reconstracted C-mass dependence vs fill in Fig. 10 and 11 on the rate dependence of the energy measurements and fill dependence of the Time of Flight measurements. These rate dependencies will be discused in more details in Section A.1.

In Section A.1 it will be confirmed that high rates lead to distortion of the energy (as well as of event rate) measurements by the $\mathrm{pC}$ polarimeters towards lower values, leaving the time of flight measurements almost unaffected. Our energy corrections from the "Dead Layer" approach partially takes care about the shift in energy measurements due to rate effect. But only partially - because the energy dependence of the "Dead layer" correction is not the same as from rate effect.

Unfortunately with the available data (including the dedicated rate effect studies during APEX sessions) we could not build a consistent quantitative picture of the dependence of the measured polarization on measured rates. One of the possible reasons for that could be that the measured rates of carbon events may not directly correlate with the total event rate in the system, which inludes prompt event rate and other backgrounds. 


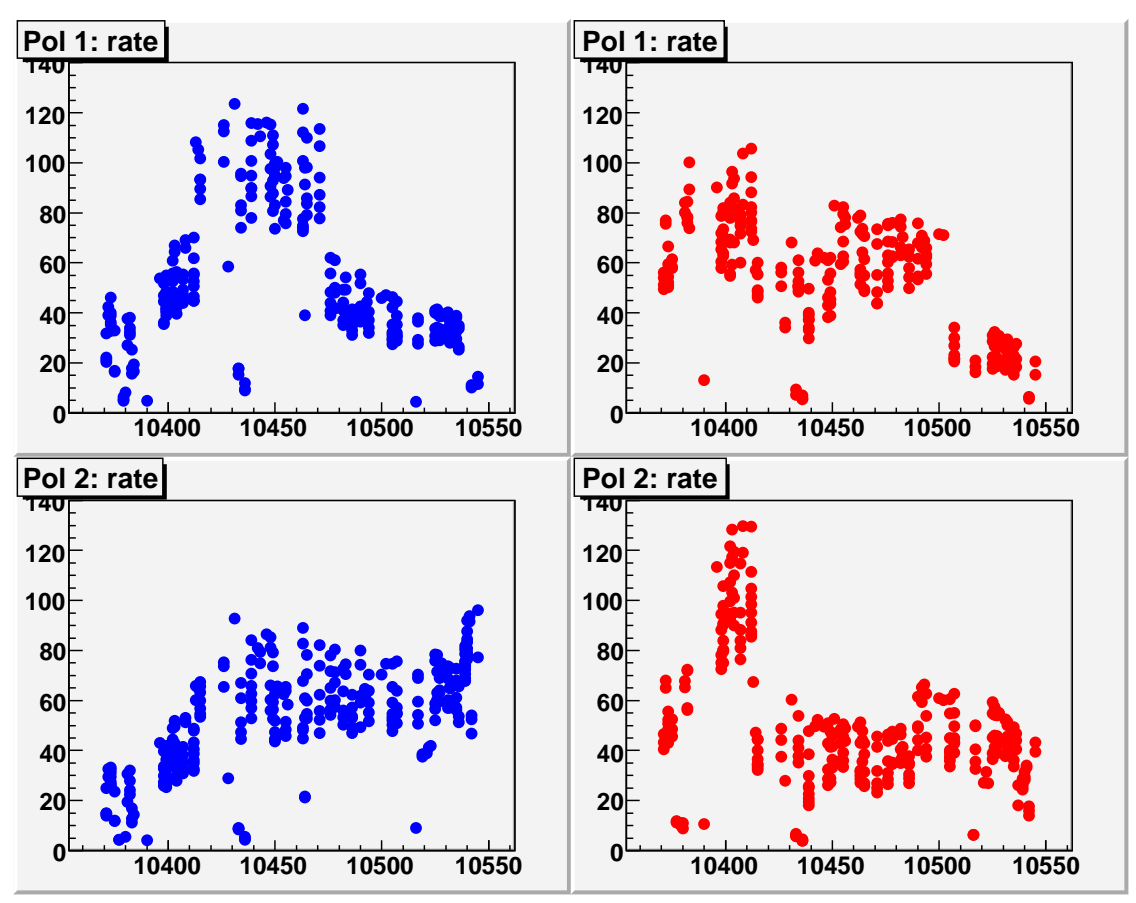

Figure 12. Rate of Carbon events per strip $(\mathrm{kHz})$ in $\sqrt{s}=500 \mathrm{GeV}$ measurements vs fill number (each fill usually had several measurements, all of them are shown here); top left - Blue1, bottom left - Blue2, top right - Yellow1 and bottom right - Yellow2.
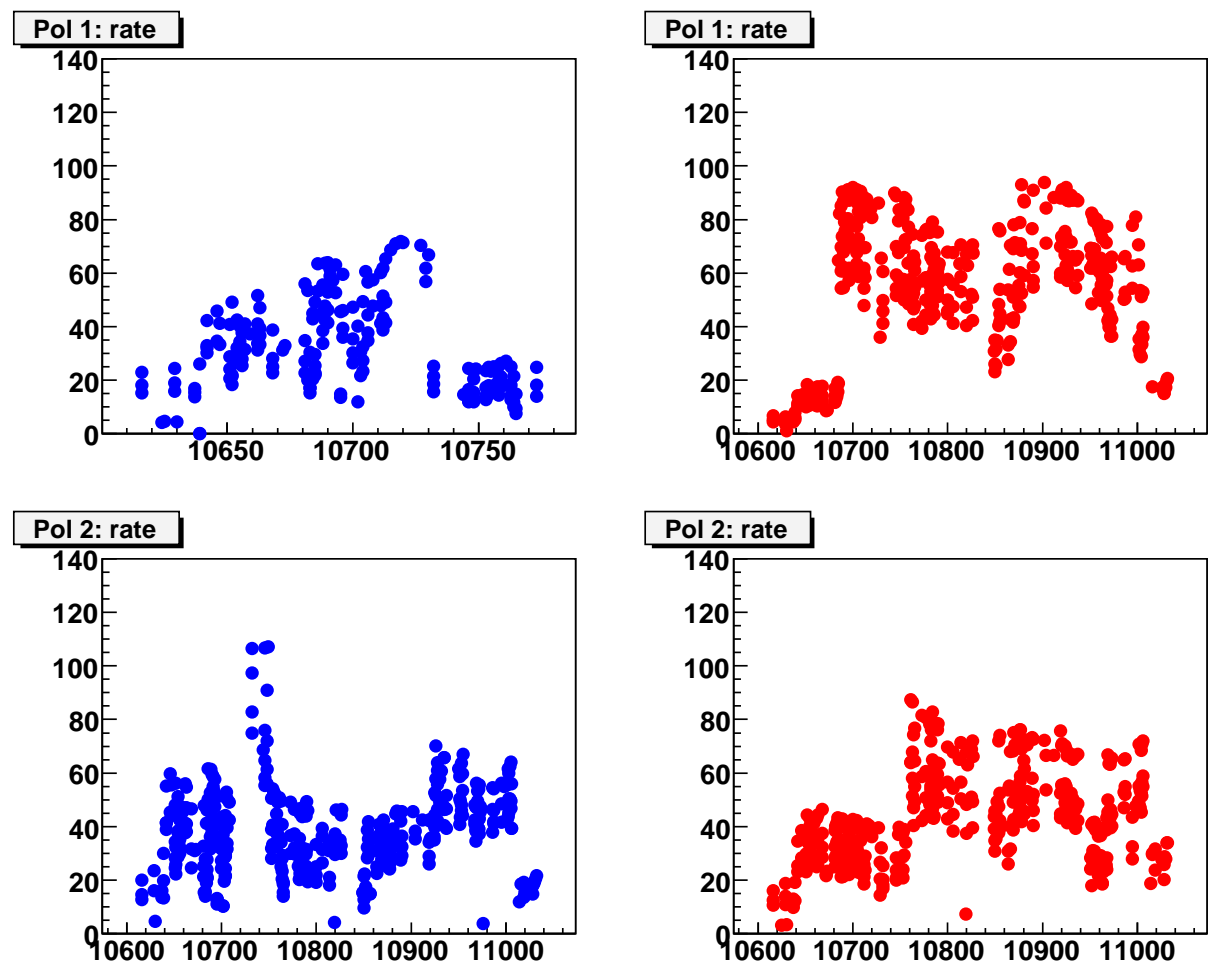

Figure 13. The same as Fig. 12, but for $\sqrt{s}=200 \mathrm{GeV}$ measurements; notice that Blue1 didn't have measurements for fills $>10773$. 

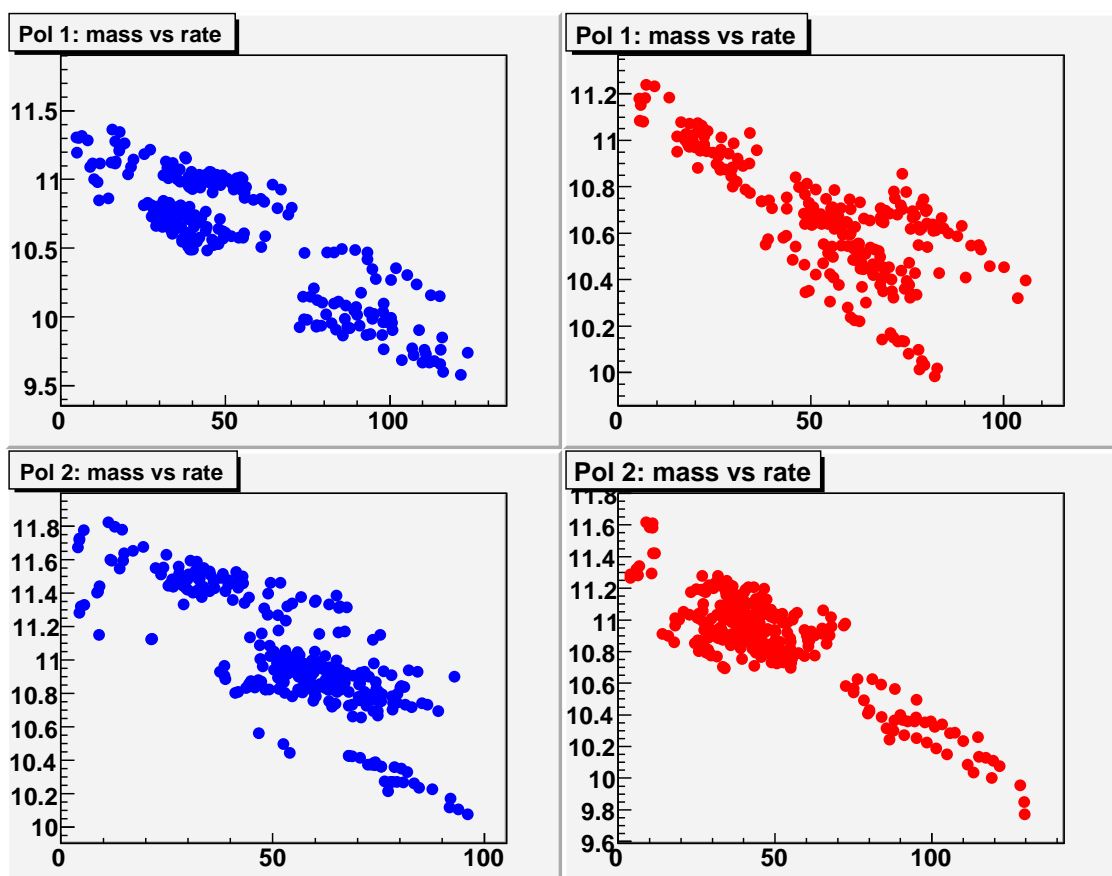

Figure 14. Reconstructed mass $\left(\mathrm{GeV} / \mathrm{c}^{2}\right)$ vs carbon event rate per strip $(\mathrm{kHz})$ in $\sqrt{s}=500$ $\mathrm{GeV}$ measurements (each fill usually had several measurements, all of them are shown here); top left - Blue1, bottom left - Blue2, top right - Yellow1 and bottom right - Yellow2.
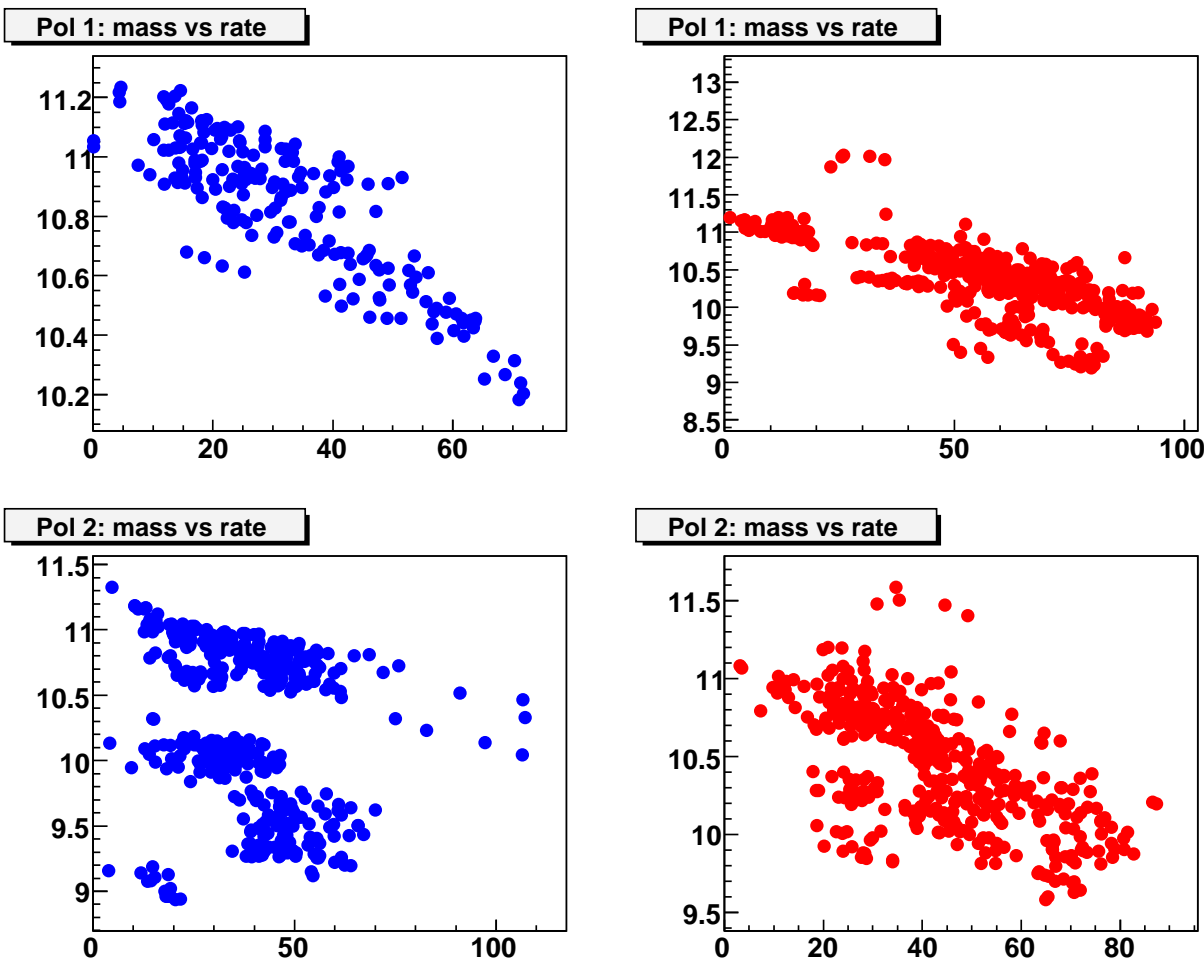

Figure 15. The same as Fig. 14, but for $\sqrt{s}=200 \mathrm{GeV}$ measurements; notice that Blue1 didn't have measurements for fills $>10773$. 


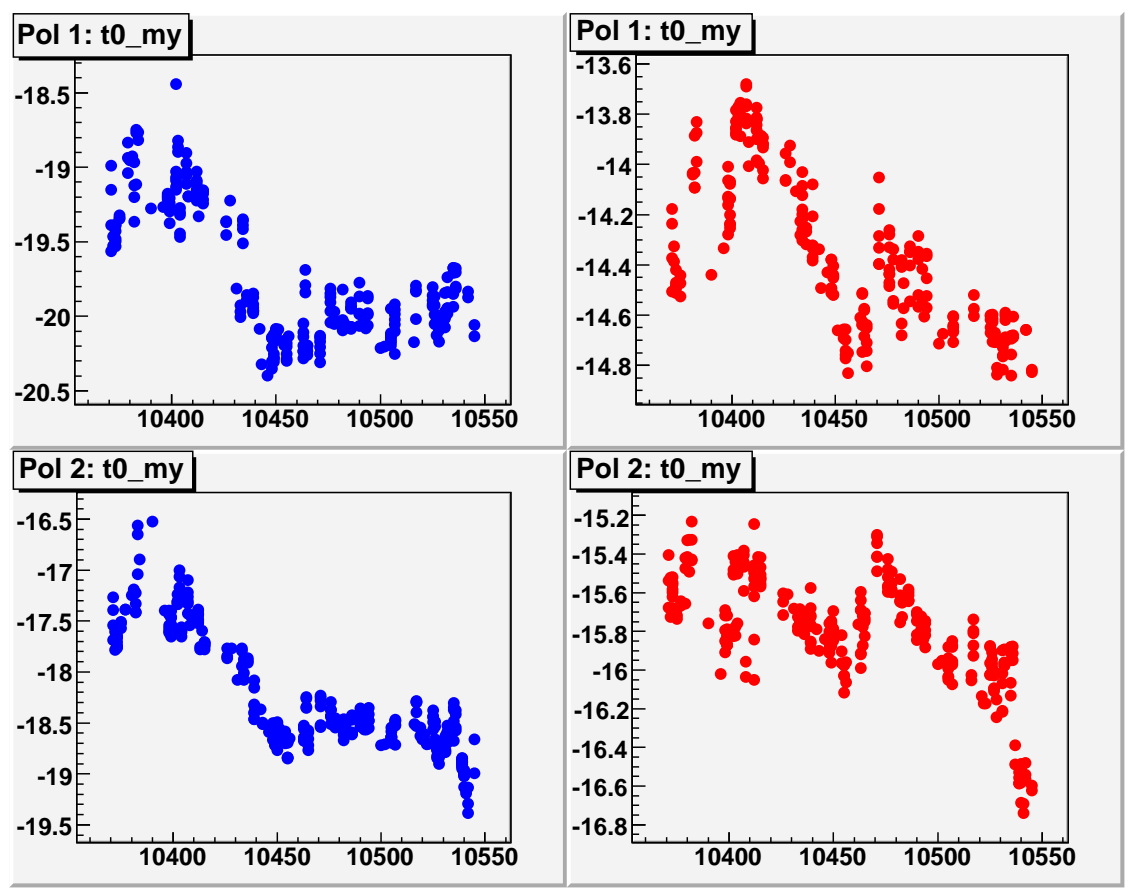

Figure 16. The average over the polarimeter strips $t 0(\mathrm{~ns})$ in $\sqrt{s}=500 \mathrm{GeV}$ measurements vs fill number (each fill usually had several measurements, all of them are shown here); top left - Blue1, bottom left - Blue2, top right - Yellow1 and bottom right - Yellow2.
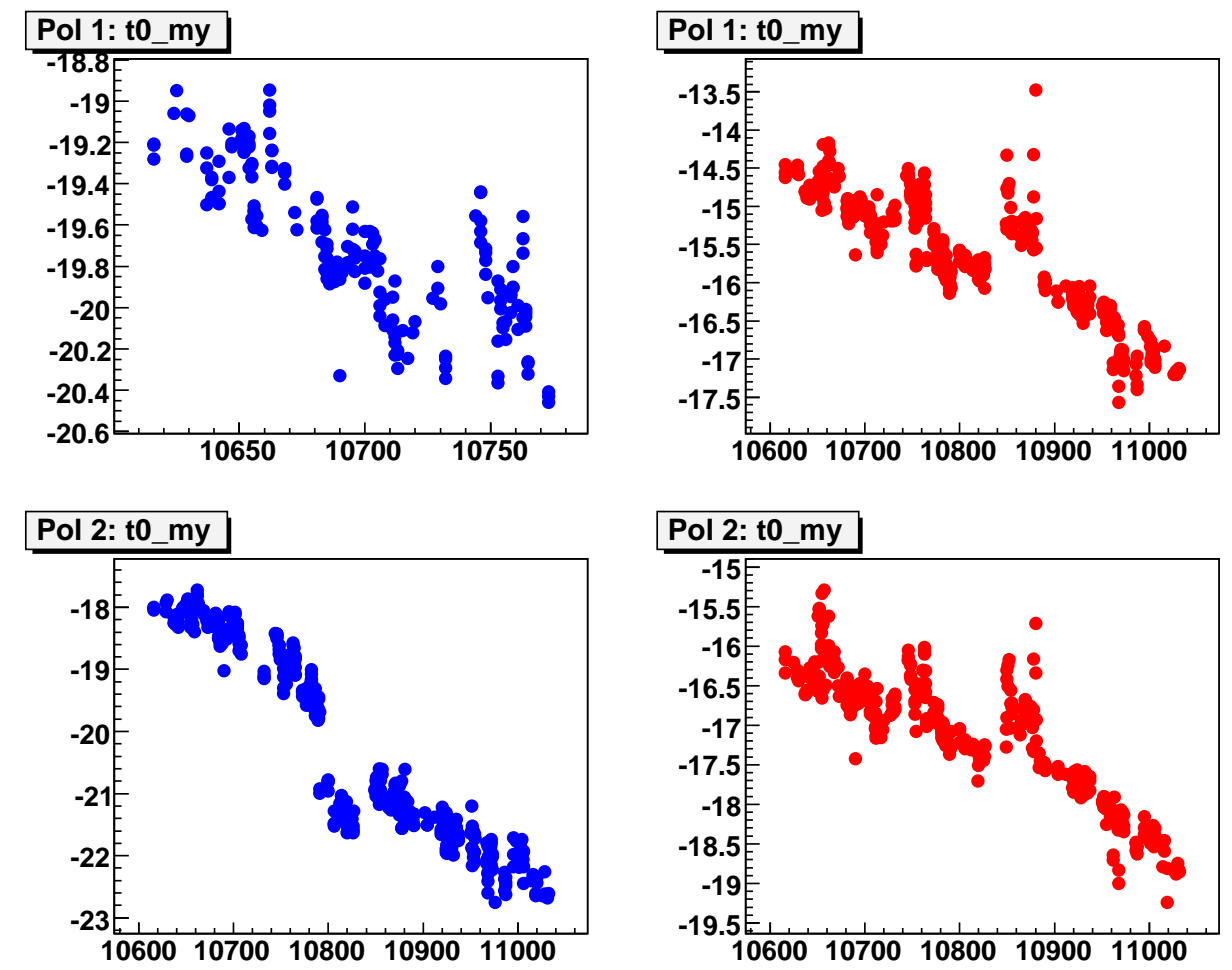

Figure 17. The same as Fig. 16, but for $\sqrt{s}=200 \mathrm{GeV}$ measurements; notice that Blue1 didn't have measurements for fills $>10773$. 

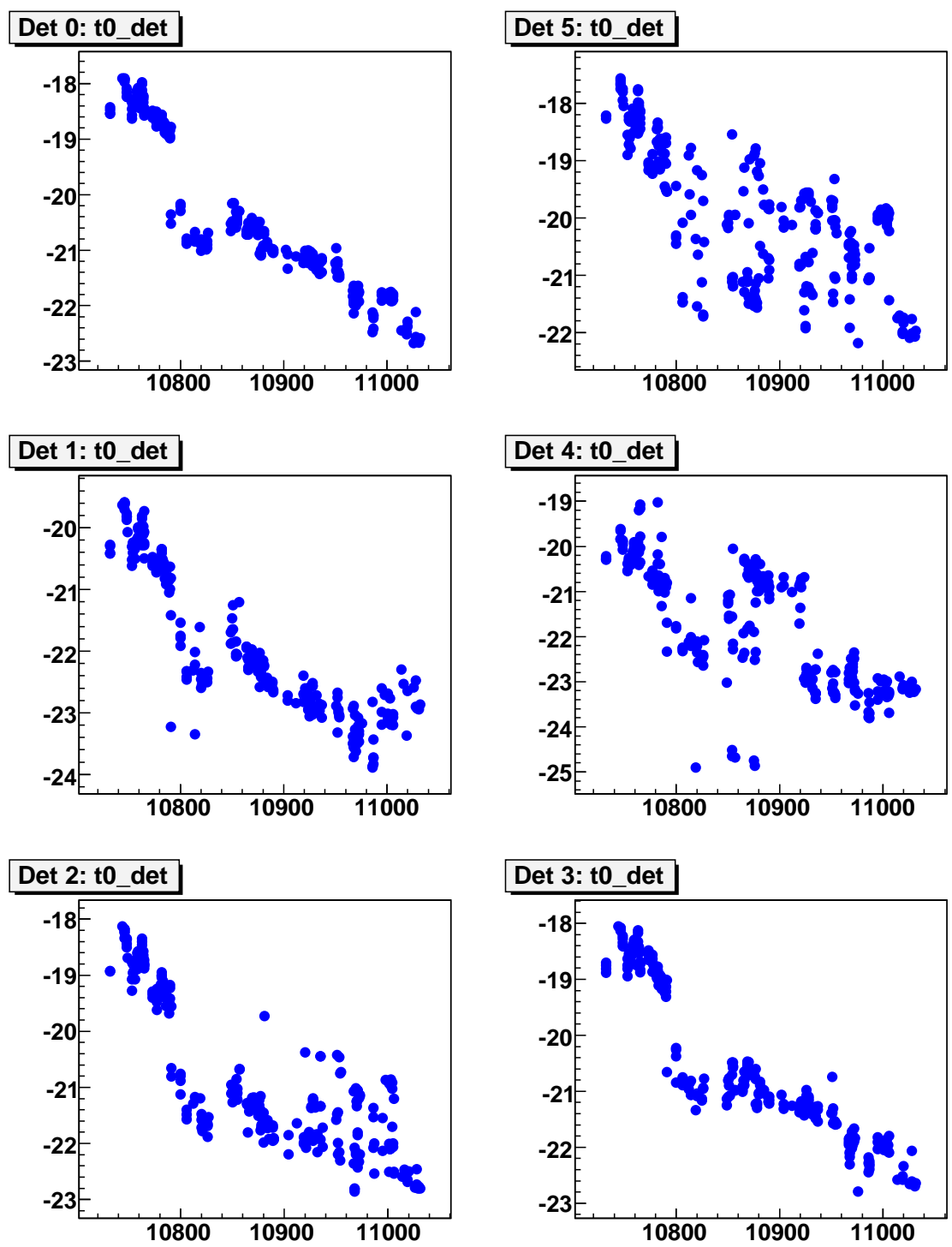

Figure 18. $t 0$ (ns) as the average over the detector strips in $\sqrt{s}=200 \mathrm{GeV}$ measurements from Blue2 vs fill number (each fill usually had several measurements, all of them are shown here); all 6 detectors are shown, detectors 1 and 4 (middle plots) being Hamamatsu strip photodiods, all others being BNL's Si detectors; only measurements with vertical target are shown. 

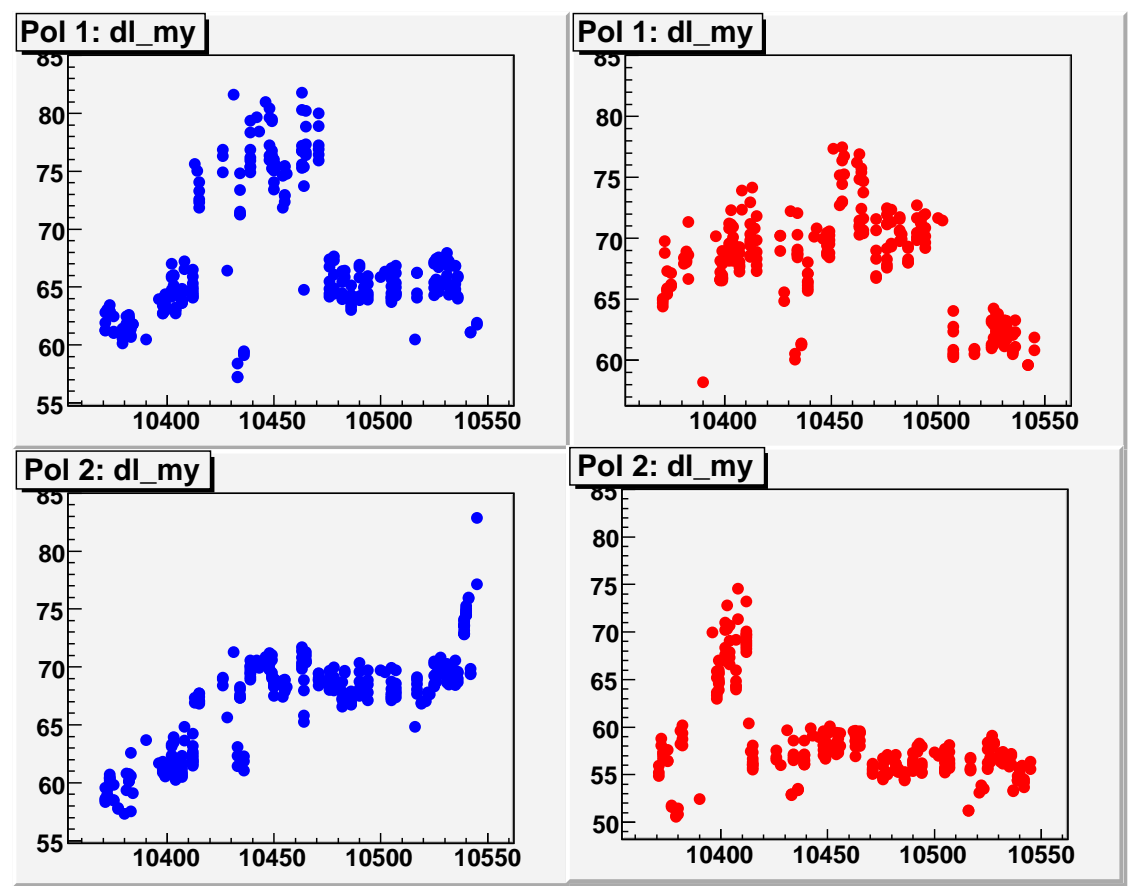

Figure 19. $x_{D L}\left(\mu \mathrm{g} / \mathrm{cm}^{2}\right)$, averaged over the polarimeter strips in $\sqrt{s}=500 \mathrm{GeV}$ measurements vs fill number (each fill usually had several measurements, all of them are shown here); top left - Blue1, bottom left - Blue2, top right - Yellow1 and bottom right - Yellow2.
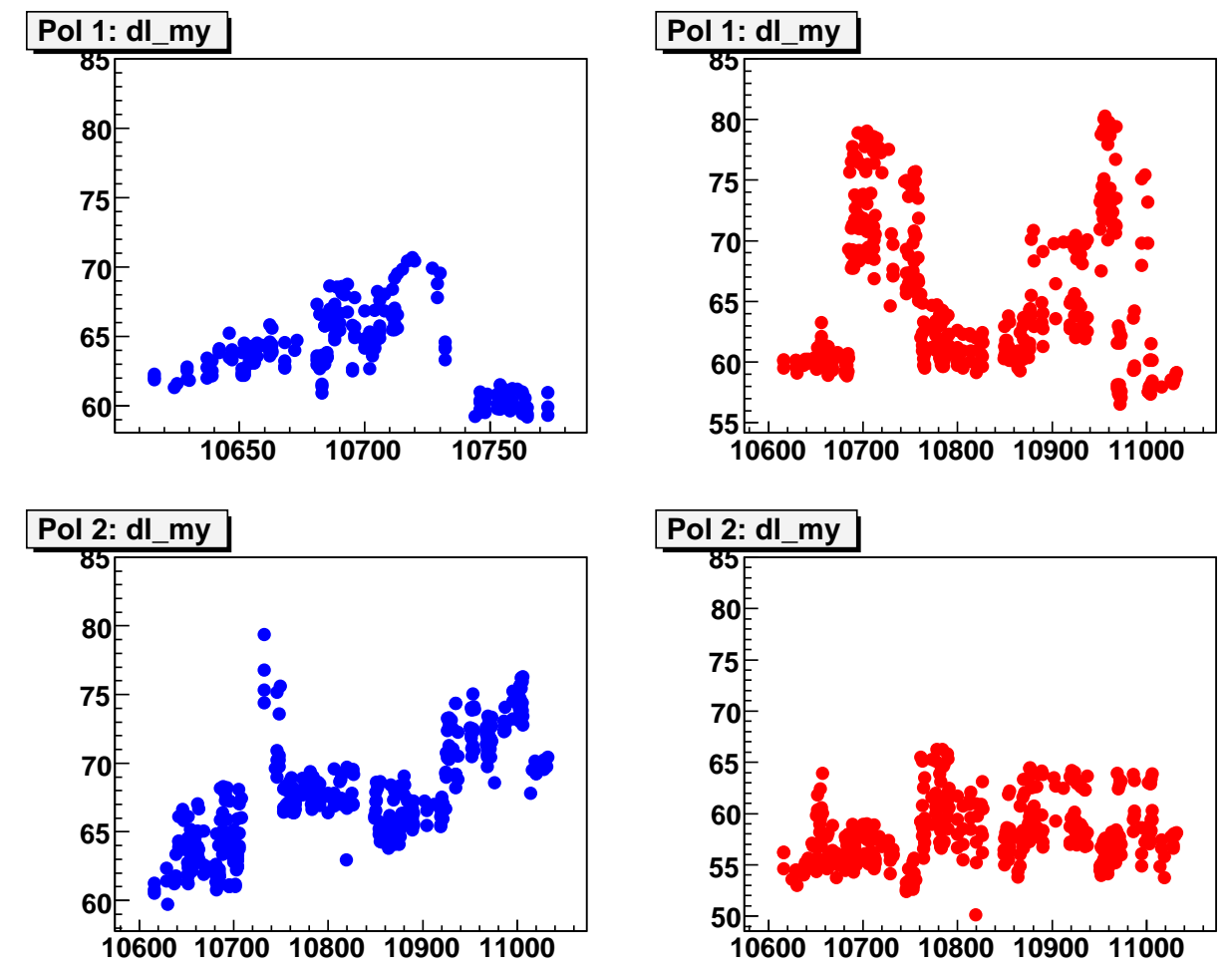

Figure 20. The same as Fig. 19, but for $\sqrt{s}=200 \mathrm{GeV}$ measurements; notice that Blue1 didn't have measurements for fills $>10773$. 

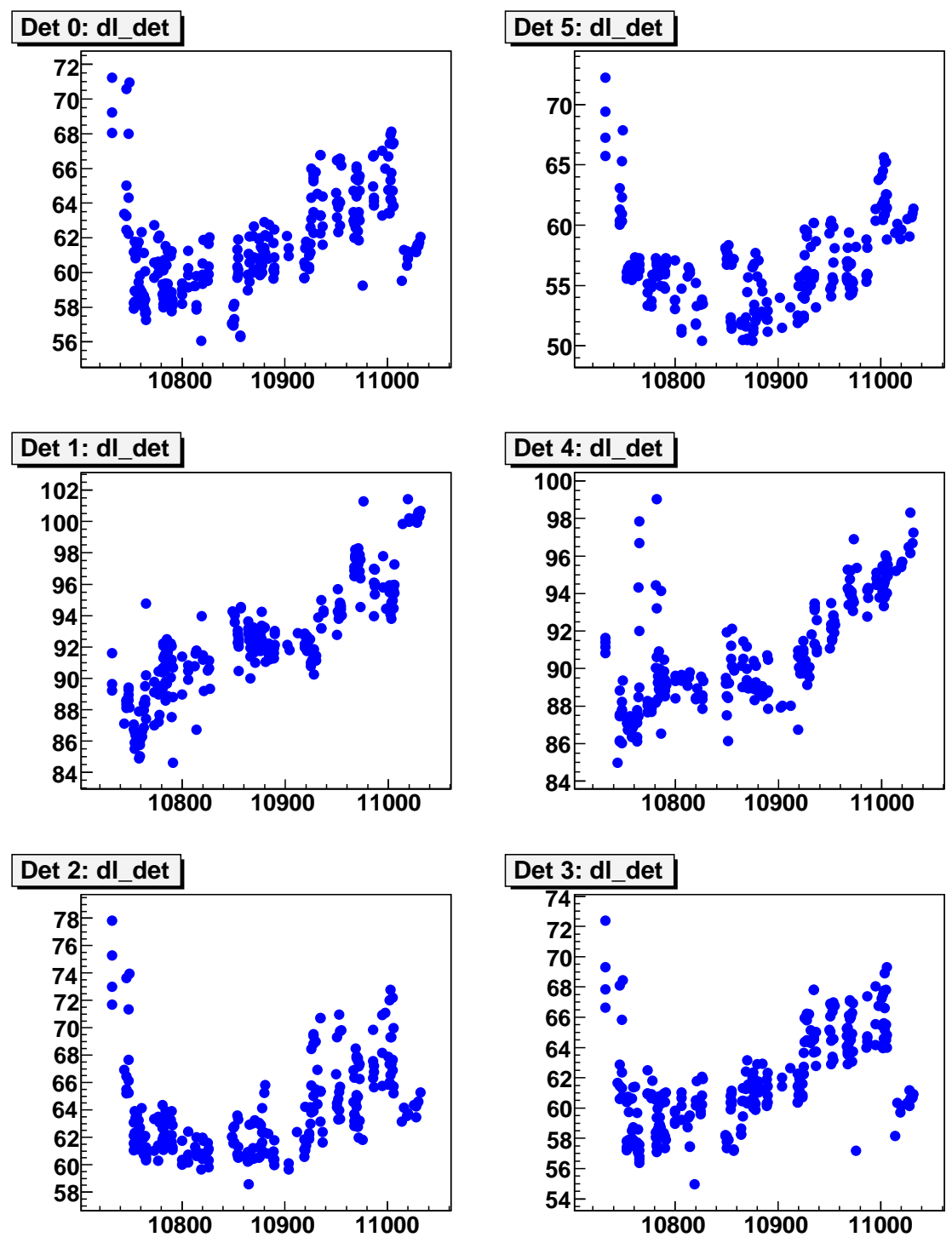

Figure 21. $x_{D L}$ (ns), averaged over the detector strips in $\sqrt{s}=200 \mathrm{GeV}$ measurements by Blue2 vs fill number (each fill usually had several measurements, all of them are shown here); all 6 detectors are shown, detectors 1 and 4 (middle plots) being Hamamatsu strip photodiods, all others being BNL's Si detectors; only measurements for vertical targets are shown. 


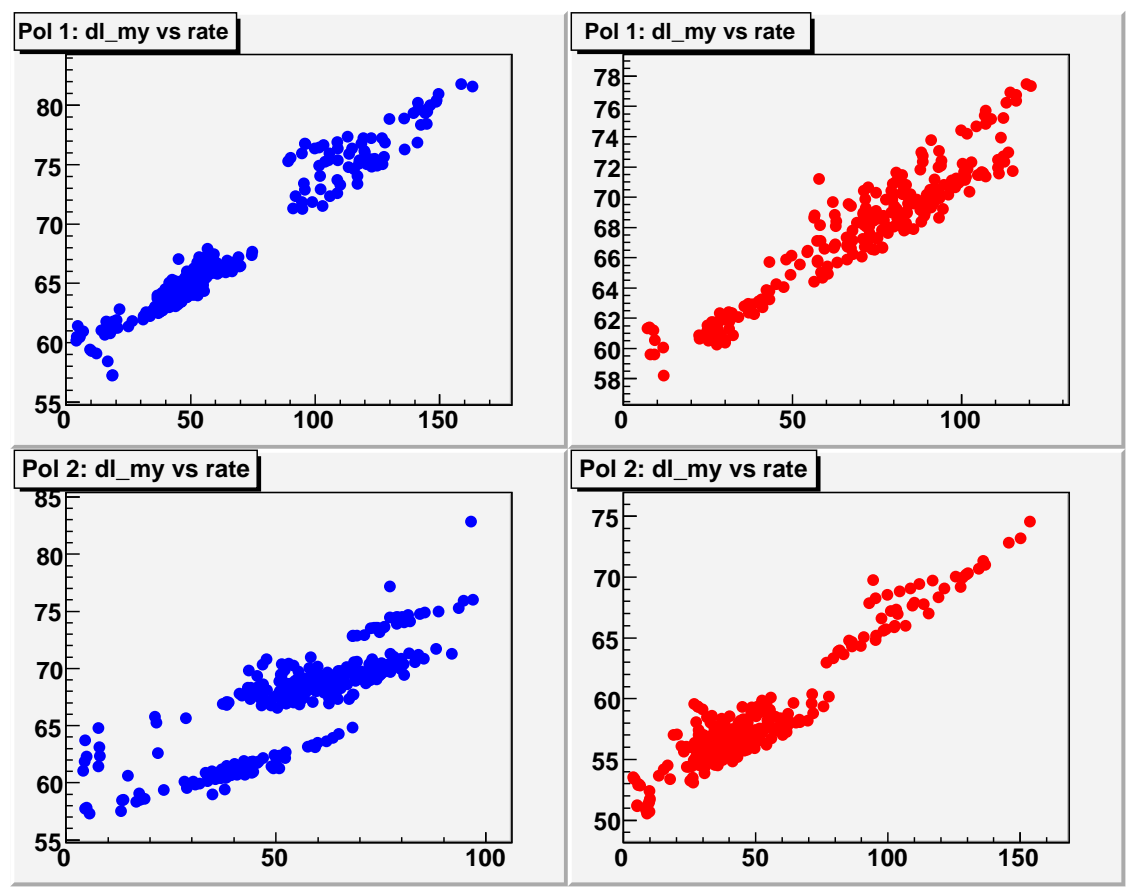

Figure 22. $x_{D L}\left(\mu \mathrm{g} / \mathrm{cm}^{2}\right)$, average over the polarimeter strips vs the carbon event rate per strip $(\mathrm{kHz})$ in $\sqrt{s}=500 \mathrm{GeV}$ measurements vs fill number (each fill usually had several measurements, all of them are shown here); top left - Blue1, bottom left - Blue2, top right - Yellow1 and bottom right - Yellow2.
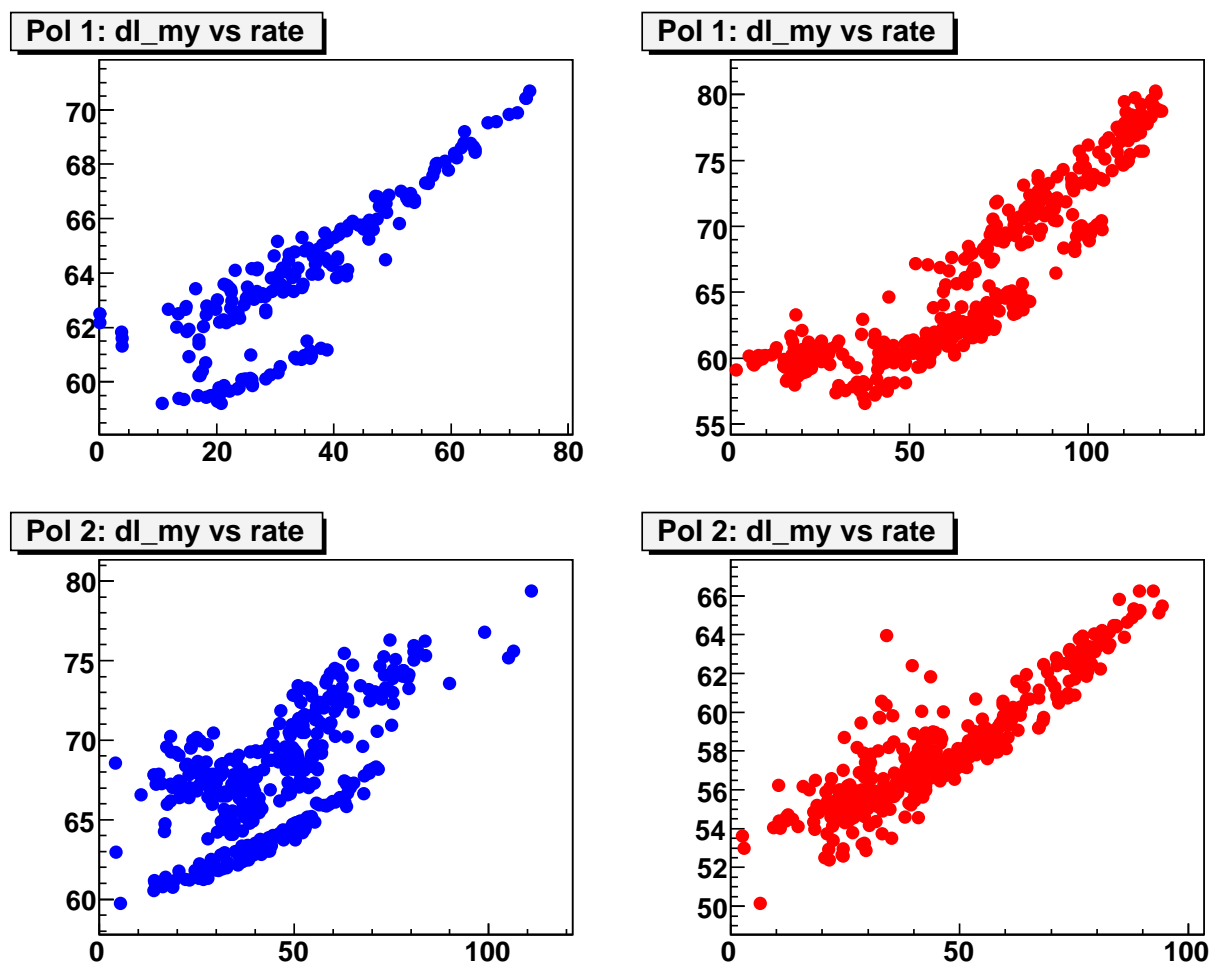

Figure 23. The same as Fig. 22, but for $\sqrt{s}=200 \mathrm{GeV}$ measurements; notice that Blue1 didn't have measurements for fills $>10773$. 


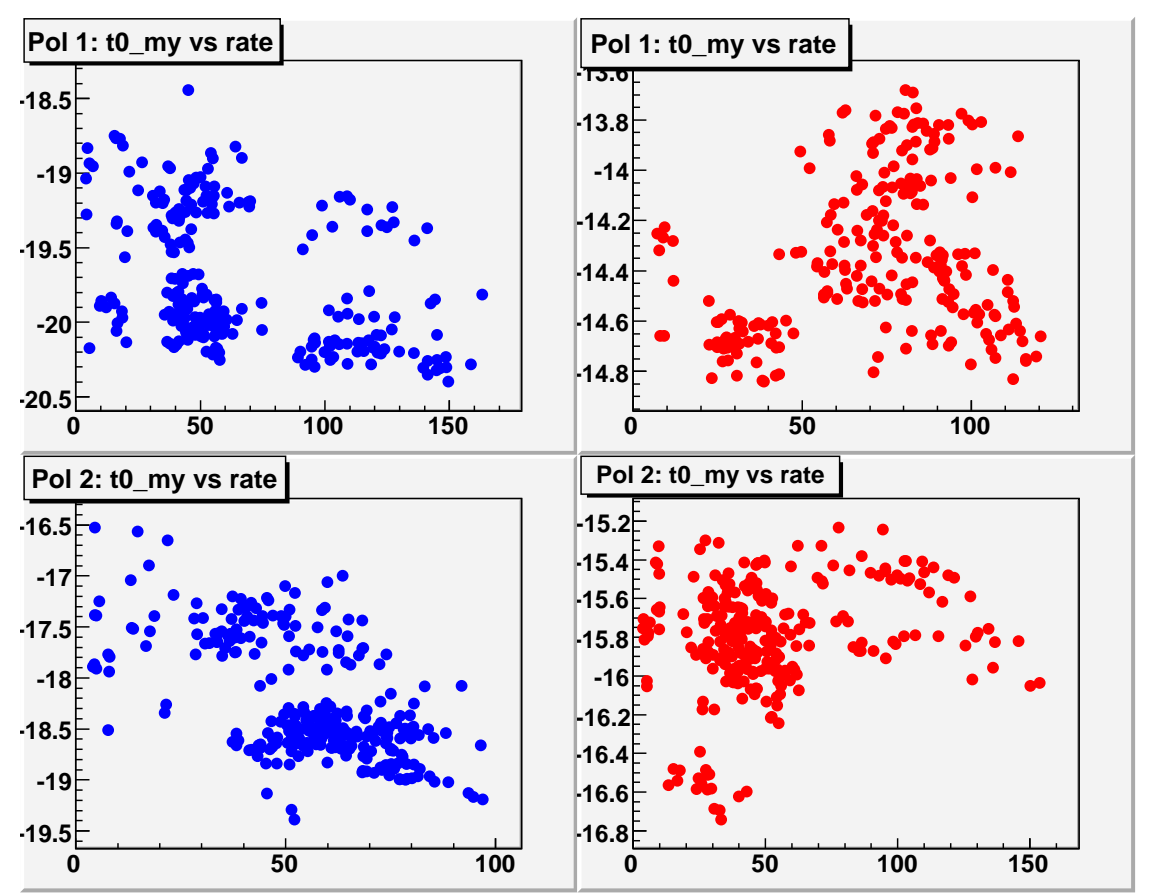

Figure 24. $t 0$ (ns) as the average over the polarimeter strips vs carbon event rate per strip $(\mathrm{kHz})$ in $\sqrt{s}=500 \mathrm{GeV}$ measurements vs fill number (each fill usually had several measurements, all of them are shown here); top left - Blue1, bottom left - Blue2, top right - Yellow1 and bottom right - Yellow2.
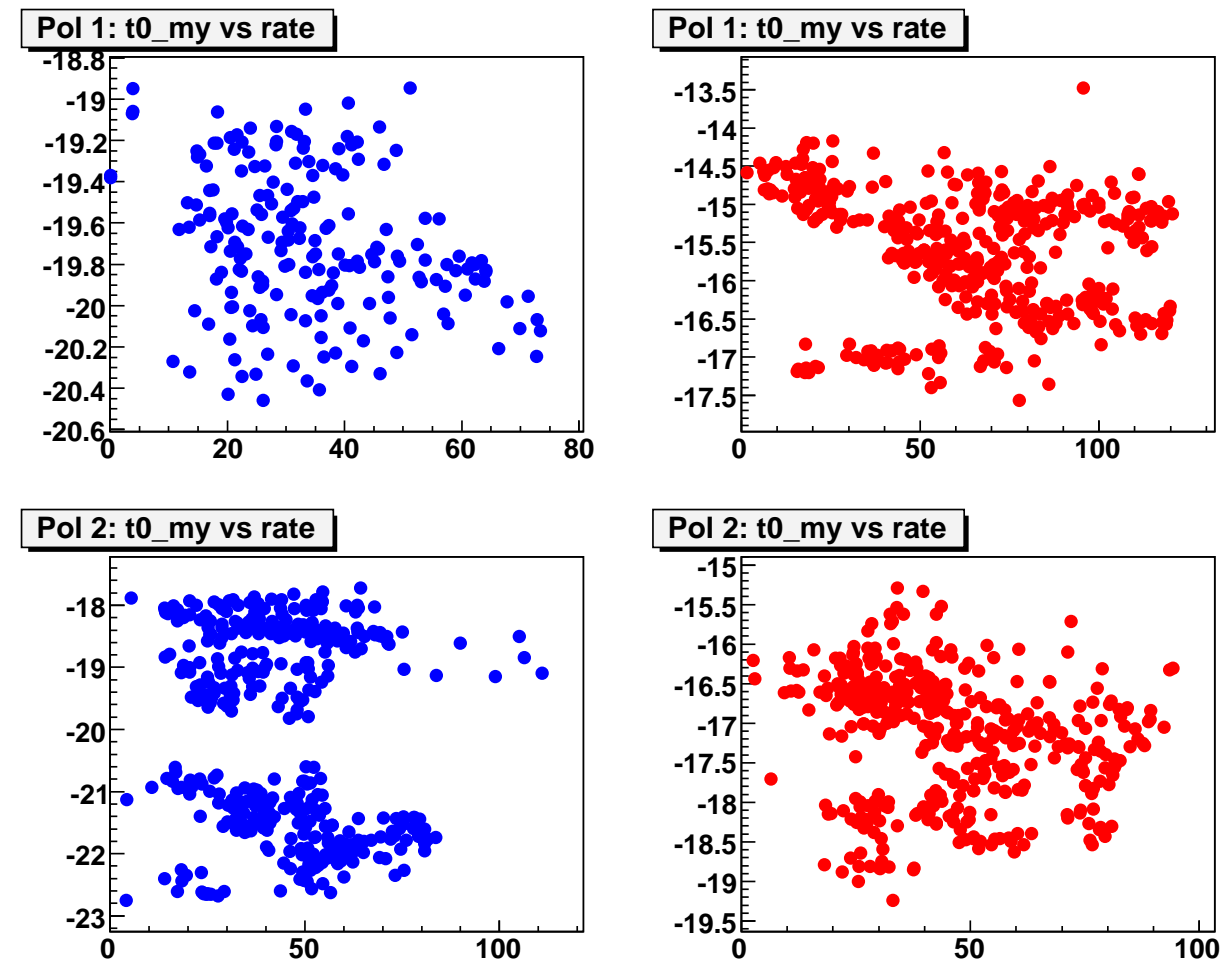

Figure 25. The same as Fig. 24, but for $\sqrt{s}=200 \mathrm{GeV}$ measurements; notice that Blue1 didn't have measurements for fills $>10773$. 
Eventually, the measured polarizations were corrected only for the effective $x_{D L}$ and $t 0$ (like we did in all previos Runs), and systematic uncertainties were derived from the residual inconsistency in the polarization measurements by Polarimeter- 1 and Polarimeter- 2 and from comparison with HJet polarimeter measurements - this will be discussed later in this note.

\section{Data Quality Assurance}

\subsection{Strip Quality Assurance}

The data quality assurance was very similar to what we used in previous year analyses. The Quality Assurance goal is to identify really abnormal strips and runs. We checked strip-by-strip:

- Number of events in the banana

- Reconstructed from the fit the effective dead layer $x_{D L}$ and $t 0$

- Reconstructed C-mass and width

- Non-constant behavior of reconstructed C-mass vs recoile carbon $E_{k i n}$ (in terms of RMS)

- The slope of recoil carbon energy distribution, $d N / d E_{k i n}$ vs $E_{k i n}$ (in terms of the slope of the fit to $\exp$ )

From the final polarization analysis we excluded the Hamamatsu detectors in Blue2 and Yellow2 polarimeters (Hamamatsu detectors from Yellow2 were not in the common data stream anyway). We did so not only to perform a more uniform analysis (with only one type of detector), but also due to too large variations in the slope of $d N / d E_{k i n}$ vs $E_{k i n}$ in the Blue2 Hamamatsu detectors, see Fig. 63 and 64.

The test of the "Number of events in the banana" helped us to discover a few very noisy channels in Blue2 of detector 1 (Hamamatsu strip) - strips 15, 17 and 19 - which affected also strips $(33,35,37),(51,53,55)$ and $(69,71,1)$, which are connected to the same WFD. All these strips were excluded from the analysis. Also from time to time Channel 33 in Blue1 showed instabilities and was excluded from many measurement analysis. The problems in channel 33 were also noticed in the alpha calibration runs (see Section 2).

Channels 53 and 55 were very noisy in Yellow1, so they were disconnected and were not used in the data analysis (and data collection). Channel 70 often in Yellow1 and occatioanally in Yellow2 showed very few (or no) events in banana cut, it was excluded from these measurements. Strip 34 in both Yellow1 and Yellow2 showed too sharp slope for Ekin distribution (roughly twice sharper than other strips), so was excluded from the final analysis.

The time of flight measurements in several WFDs in yellow polarimeters in fills 10850 and 10851 showed a jump by $\sim 8$ ns (and after that back to normal). Besides that data didn't show any other abnormalities, after accounting for this time offset the data was used in the final anaysis. 
Table 1 showes a summary of major problems discovered during the strip QA procedure. In addition to the masked strips mentioned in the table some measurements had 1-2 more strips masked due to different reasons (listed in the beginning of this section).

Table 1

QA summary

\begin{tabular}{|c|c|c|c|}
\hline Strip(s) & Problem & Pol@ $\sqrt{s}$ & Masked \\
\hline 33 & Instabilities in amp & Blue1@200GeV & occasionally \\
\hline Det 1 and 4 & $E_{k i n}$ slope & Blue2@200GeV & all measurements \\
\hline $33,35,37,51,53,55,69,71,1$ & Because of noisy strips $15,17,19$ & Blue2@200GeV & all measurements \\
\hline 53,55 & Too noisy & Yell1@200GeV & all measurements \\
\hline 70 & Too few $\mathrm{C}$ events & Yell1@200GeV & occasionally \\
\hline 34 & $E_{k i n}$ slope & Yell1@200GeV & all measurements \\
\hline 70 & Too few $\mathrm{C}$ events & Yell2@200GeV & occasionally \\
\hline 34 & $E_{k i n}$ slope & Yell2@200GeV & all measurements \\
\hline 33 & Instabilities in amp & Blue1@500GeV & occasionally \\
\hline Det 1 and 4 & $E_{k i n}$ slope & Blue2@500GeV & all measurements \\
\hline $33,35,37,51,53,55,69,71,1$ & Because of noisy strips $15,17,19$ & Blue2@500GeV & all measurements \\
\hline 53,55 & Too noisy & Yell1@500GeV & all measurements \\
\hline 70 & Too few $\mathrm{C}$ events & Yell1@500GeV & occasionally \\
\hline 34 & $E_{k i n}$ slope & Yell1@500GeV & all measurements \\
\hline 70 & Too few $\mathrm{C}$ events & Yell2@500GeV & occasionally \\
\hline 34 & $E_{k i n}$ slope & Yell2@500GeV & all measurements \\
\hline
\end{tabular}

\subsection{Bunch Quality Assurance}

As in previous years we didn't use bunch 20 in our analysis, because it was used for RHIC fill setup and tune, so it usually showed a different behavior (emittance etc.) compared to the other bunches. Bunch 0 was also masked in our analysis because it was used to inject generator pulses in the system for monitoring purposes. Due to instabilities of the amplitude and time position of the pulses they sometimes overlapped with Carbon events in the banana.

Unlike in previous years, in this analysis we didn't reject measurements based on our usual control of the consistency of the bunch-by-bunch asymmetry and "specific" luminosity (bunch event rate normalized by bunch intensity). This inconsistency (in the first bunches compared to other bunches) used to show up in the past when a very thick target was accidently put in the beam. In 2009, particularly for the $\sqrt{s}=500 \mathrm{GeV}$ data we had very often such an inconsistency due to high event rates in the polarization measurements (on the average $\sim 2-4$ times higher than in previous years). An example of such a measurement is shown in Fig. 59 and 60. As will be discussed in Section A.1, this inconsistency may come from a bunch dependence of the rate effect and a slightly different response of detectors to high rates, which lead to bunch dependent left-right or up-down 
detector asymmetries. Since the average over bunches detector asymmetry in our analysis is properly canceled in the physics asymmetries, this inconsistency in the asymmetry measurements between bunches should not directly affect the polarization results. This was confirmed in a few measurements when we compared polarizations measured in the first 20 bunches (where the effect is maximal) vs last 20 bunches (where there is no effect) and found consistency within statistical uncertainties.

\section{Polarization measurements}

\subsection{Polarimeters-1 vs Polarimeters-2}

Fig. 26 and 27 show the comparison of polarization measurements by Polarimeter-1 and Polarimeter- 2 in blue and yellow rings. It is particularly obvious for $\sqrt{s}=500 \mathrm{GeV}$, the variation of the ratio of the measurements by two polarimeters is not statistical. If points on bottom plots are projected on the vertical axis the Mean/RMS are 0.96/0.09 and $0.92 / 0.11$ for blue and yellow for $\sqrt{s}=500 \mathrm{GeV}$ and $0.972 / 0.049$ and 1.000/0.059 for blue and yellow for $\sqrt{s}=200 \mathrm{GeV}$. These inconsistencies, $\sim 12 \%$ for $\sqrt{s}=500 \mathrm{GeV}$ and $\sim 6 \%$ for $\sqrt{s}=200 \mathrm{GeV}$ will be included in the fill-by-fill systematic uncertainties in our measurements. The online polarization values gave slightly larger discrepancies between measurements in two polarimeters: $0.90 / 0.09$ and $0.87 / 0.12$ for blue and yellow for $\sqrt{s}=500$ $\mathrm{GeV} 0.917 / 0.052$ and $0.953 / 0.074$ for blue and yellow for $\sqrt{s}=200 \mathrm{GeV}$. This means that applied energy corrections partially cancel the systematics in our measurements.

\subsection{Polarization profile}

The polarization profile is one of the key issues in the proton beam polarization measurements at RHIC. Scanning a carbon ribbon target across the beam allows to measure beam intensity and polarization profiles in both vertical and horizontal directions in transverse plane. For a non-flat polarization profile the beam polarization will be seen differently by H-Jet polarimeter, pC polarimeter (in fixed target measurement mode) and when colliding beams in the interaction regions of RHIC experiments. The jet target at the H-Jet polarimeter is much wider than the beam width, such the polarization profile is weighted with the beam intensity profile in the average beam polarization. For colliding beams, the polarization profile is weighted with a product of two beam intensity profiles in the transverse plane. The polarization measurement in $\mathrm{pC}$ polarimeter will depend on the positioning of the carbon target, and whether a horizontal or vertical target is used for the measurements (in case the horizontal and vertical beam profiles are different).

Assuming gaussian profiles $(I(x, y)$ for intensity and $P(x, y)$ for polarization), the average beam polarization seen by H-Jet polarimeter can be expressed by:

$\langle P\rangle_{\text {HJet }}=\frac{\iint P(x, y) I(x, y) d x d y}{\iint I(x, y) d x d y}=\frac{P_{\max 2}}{\sqrt{\left(1+R_{X}\right) \cdot\left(1+R_{Y}\right)}}$.

The average beam polarization seen by pC polarimeter for the case with a vertical target positioned at maximum beam intensity (and polarization) along $\mathrm{X}$ axis:

$$
\langle P\rangle_{p C-\max X}=\frac{\int P(y) I(y) d y}{\int I(y) d y}=\frac{P_{\max 2}}{\sqrt{\left(1+R_{Y}\right)}},
$$




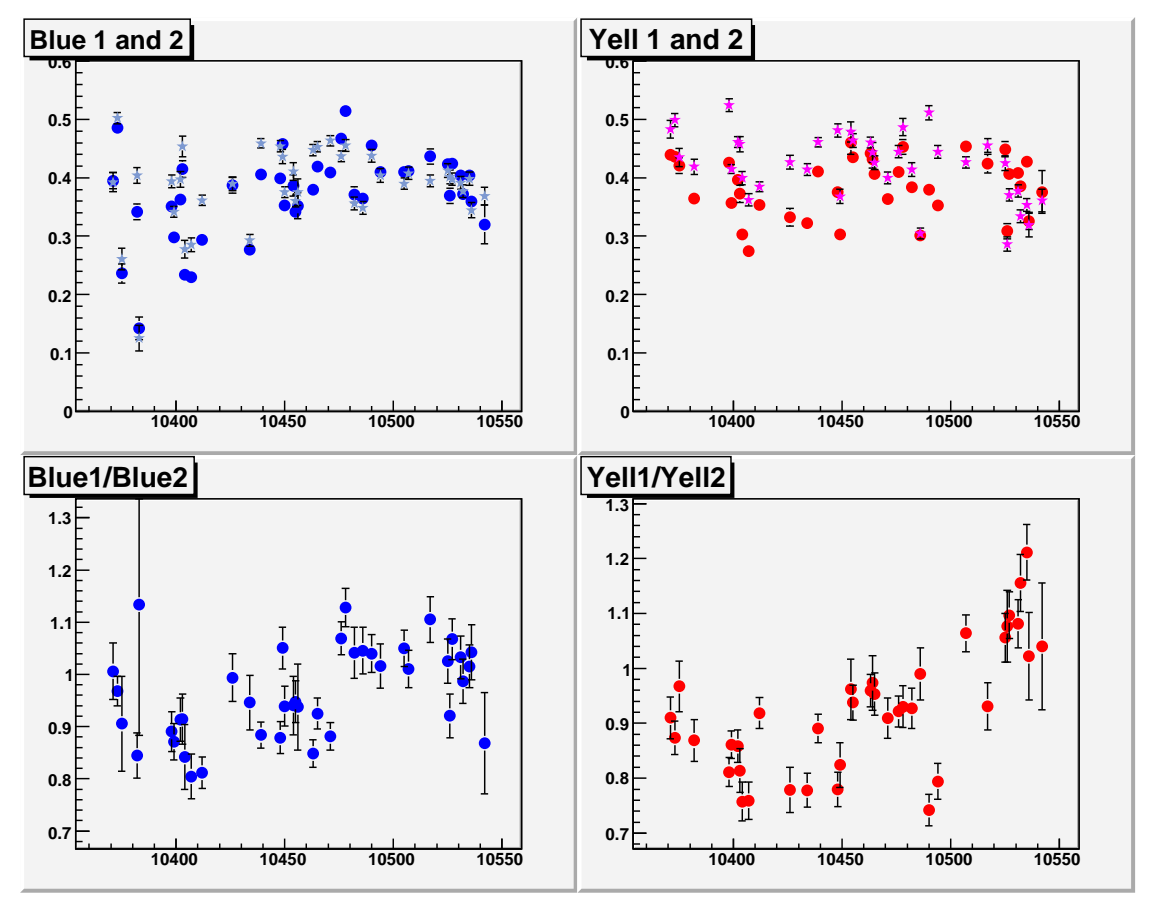

Figure 26. Fill-by-fill polarizations (weighted average over measurements in a fill) during $\sqrt{s}=500 \mathrm{GeV}$ part of Run9 (neither yet normalized to HJet nor corrected for pol. profile), by Blue1 (blue circles) and Blue2 (light blue starts) on top left, by Yellow1 (red circles) and Yellow2 (pink starts) on top right; fill-by-fill comparison of polarization measurements: Blue1/Blue2 on bottom left, and Yellow1/Yellow2 on bottom right.
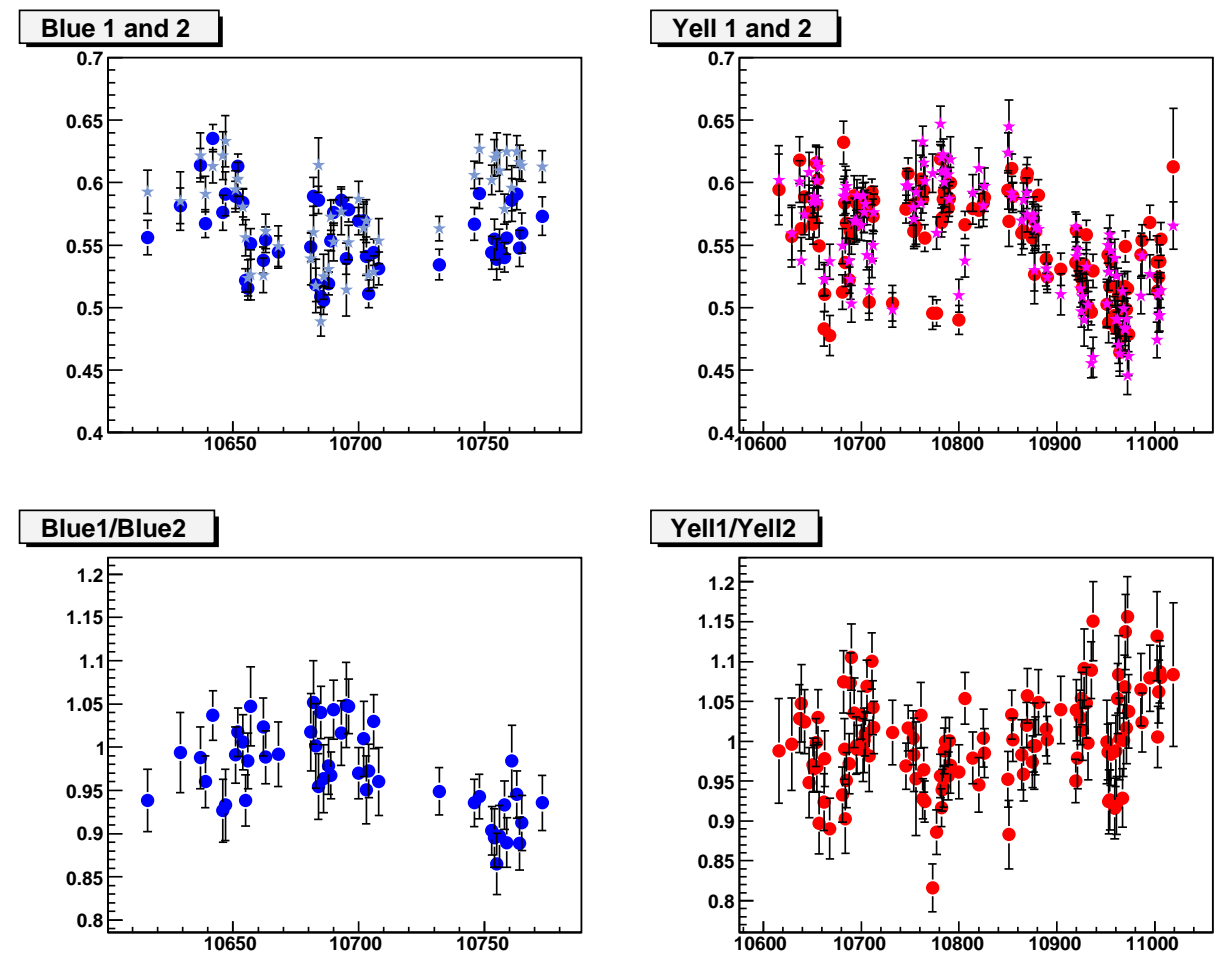

Figure 27. The same as Fig. 26, but for $\sqrt{s}=200 \mathrm{GeV}$ measurements; notice that Blue1 didn't have measurements for fills $>10773$. 
and similarly for $\langle P\rangle_{p C-\max Y}$ using a horizontal target; and for the experiments for two beam collisions, $I_{1,2}$ relating the intensity profiles for two beams, respectively:

$$
\langle P\rangle_{E x p}=\frac{\iint P(x, y) I_{1}(x, y) I_{2}(x, y) d x d y}{\iint I_{1}(x, y) I_{2}(x, y) d x d y}=\frac{P_{\max 2}}{\sqrt{\left(1+\frac{1}{2} R_{X}\right) \cdot\left(1+\frac{1}{2} R_{Y}\right)}},
$$

with $P_{\max 2}$ the polarization at beam maximum intensity and polarization in transverse plane (in 2-dim), $R_{X}$ and $R_{Y}$ the squared ratio of the intensity profile width and polarization profile width $\left(\sigma_{I} / \sigma_{P}\right)^{2}$, for $X$ and $Y$ projections, respectively.

These relations between average polarizations are taken into account when normalizing $\mathrm{pC}$ measurements to H-Jet absolute polarization measurements and when providing polarization values for RHIC experiments.

The profile parameters $R_{X}$ and $R_{Y}$ can be extracted from the direct measurements of $\sigma_{I}$ and $\sigma_{P}$ in case the profile measurements are not corrupted. Currently we see two major effects, which corrupt the measured profiles. One is the rate effects, which are clearily seen in Run9 when event rates were high (see for example Fig. 29). Another one is observed in very low rate conditions starting from Run6 (when we first started measuring profiles in each physics fill), an example from Run9 is shown in Fig. 30. According to our current understanding, this effect is seen in cases a loose (usually thin) target is used, which is attracted to the beam center when it is moved into the beam, so that effectively the target is stuck in the beam center instead of moving across the beam with uniform speed. In this case to extract the profile parameter $R$ the correlation between polarization and intensity in each target position can be used (for gaussian intensity and polarization profiles):

$\frac{P}{P_{\max }}=\left(\frac{I}{I_{\max }}\right)^{R}$

this doesn't require a knowledge on the target position. $I_{\max }$ and $P_{\max }$ are beam intensity and polarization, respectively, when the target is positioned in the beam center. Notice, $I_{\max }$ and $P_{\max }$ here are in 1-dim space (either in $\mathrm{X}$ or $\mathrm{Y}$ ), because they are averaged in the other transverse direction; $P_{\max }$ and $R$ here carry the meaning of $\langle P\rangle_{p C-\max X}$ and $R_{X}$ or $\langle P\rangle_{p C-\max Y}$ and $R_{Y}$ depending on the target orientation. From the fit to data we extract parameters $P_{\max }$ and $R$, from which we can get the average polarization across the beam (to be compare to HJet measurements, when determining the normalization for $\mathrm{pC}$ measurements):

$\langle P\rangle=\frac{P_{\max }}{\sqrt{1+R}}$

This is the same approach used in analyses of the previous years. Fig. 31 and 32 show the fill by fill measurement of the profile parameter $R$ using the polarization-intensity correlation.

Currently we do not have a clear view how to correct for the rate effect on profile measurements, which tend to give a bias to a flatter polarization profile as it is seen in Fig. 33 and to smaller extent in Fig. 34 (parameter $R$ gets closer to 0 ). If we assume that the polarization profile parameter $R$ is properly reconstraucted at low rates, we can rely on the measurements of $R$ near rate $\sim 0$, which was $\sim 0.4$ for $250 \mathrm{GeV}$ beams and $\sim 0.08$ 


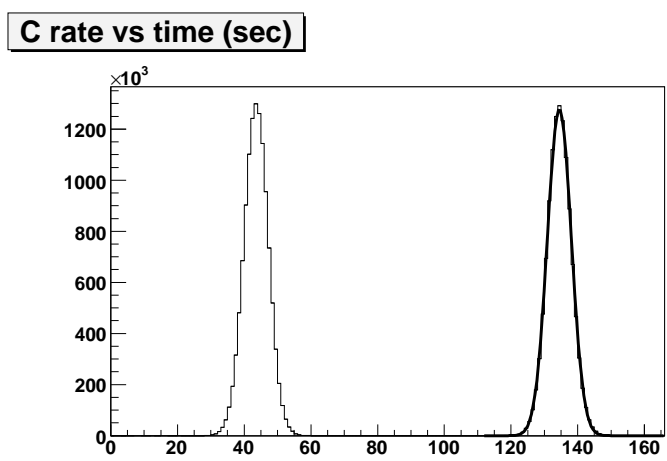

Figure 28. Measurement 10616.005 (Blue1 at $\sqrt{s}=200 \mathrm{GeV}$ ): event rate in the "banana" in $1 \mathrm{sec}$ bins; fit of the second peak with a gaussian is shown for the comparison; this is an example for a good profile

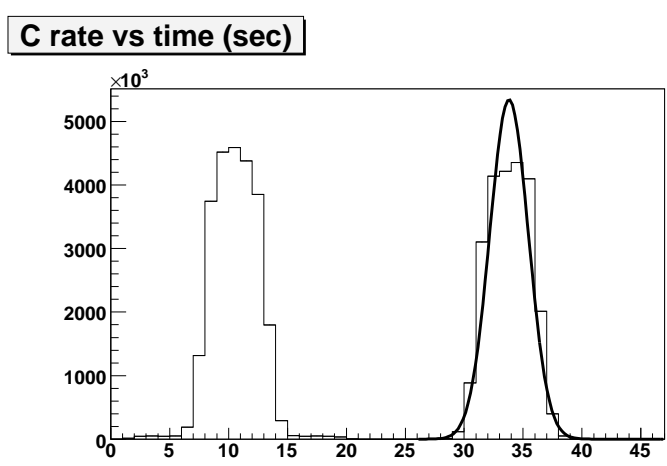

Figure 29. The same as Fig. 29, but for the measurement 10689.005 (Blue1 at $\sqrt{s}=200$ $\mathrm{GeV})$; this is an example for high rate.

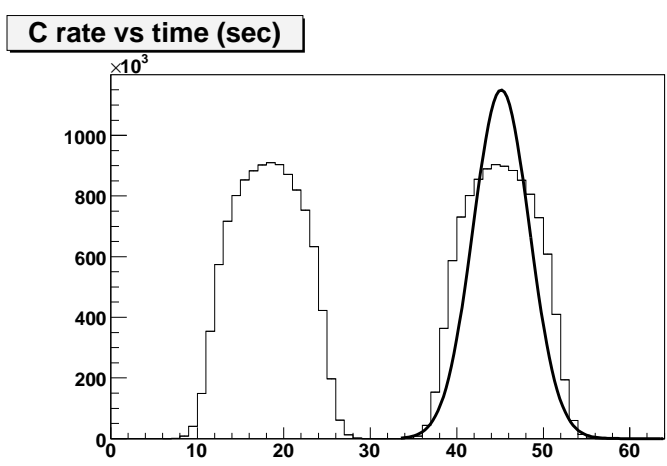

Figure 30. The same as Fig. 29, but for the measurement 10616.206 (Blue2 at $\sqrt{s}=200$ $\mathrm{GeV})$; this is an example for low rate. 


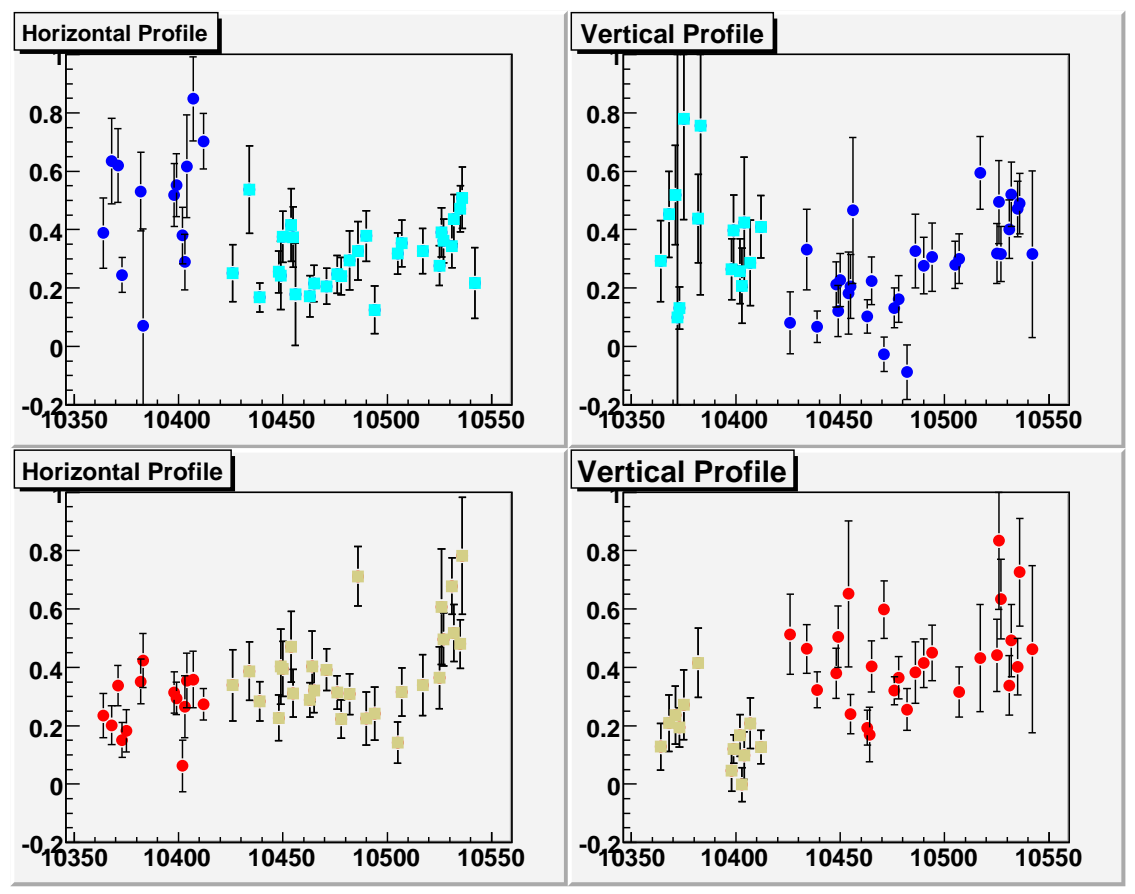

Figure 31. Polarization profile parameter $R$ vs fill for $\sqrt{s}=500 \mathrm{GeV}$ data: left plots for horizontal profile, right plots for vertical profile, upper plots for blue ring, botoom plots for yellow ring; circles for polarimeter-1, squares for polarimeter-2.
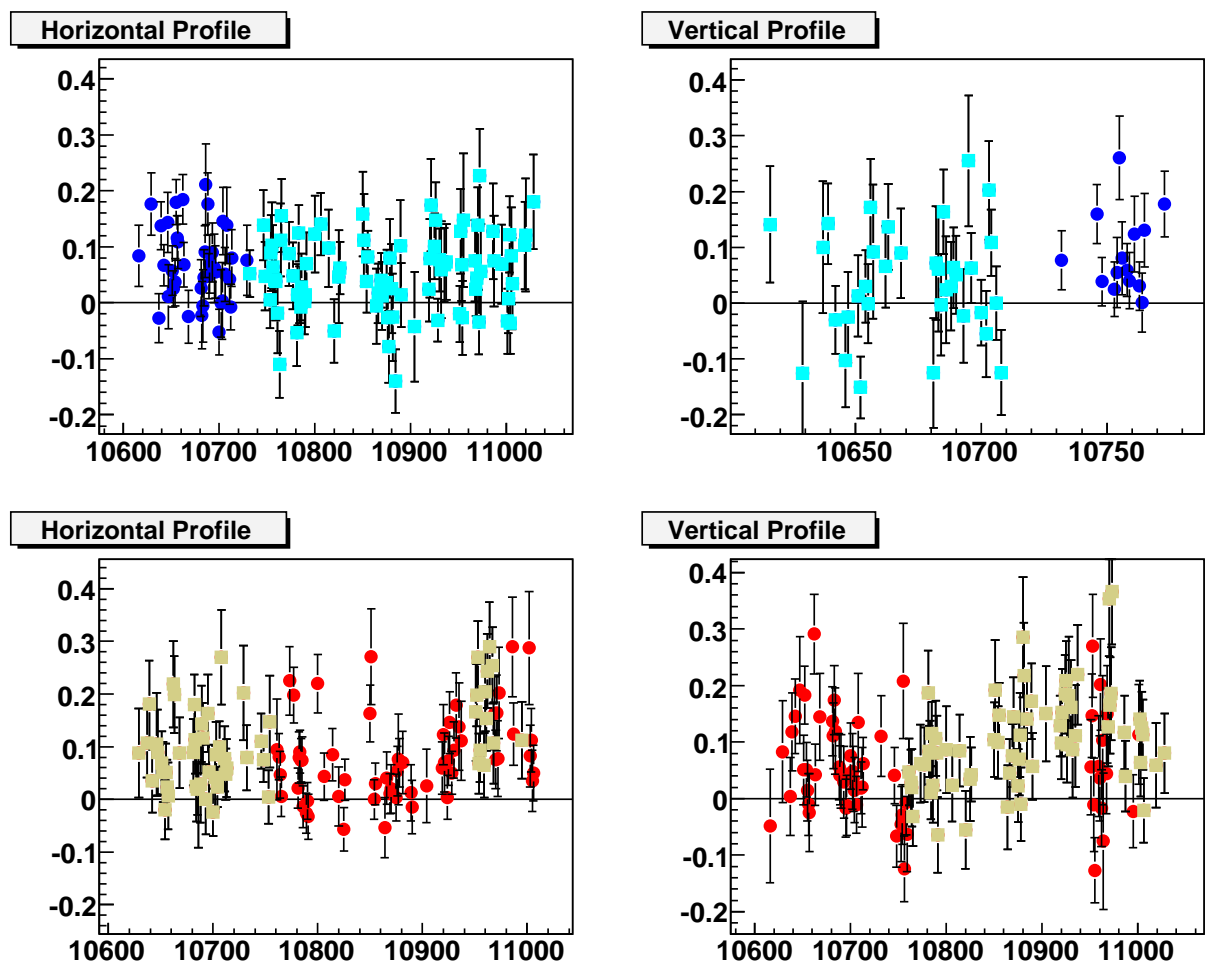

Figure 32. The same as Fig. 31, but for $\sqrt{s}=200 \mathrm{GeV}$ data. 

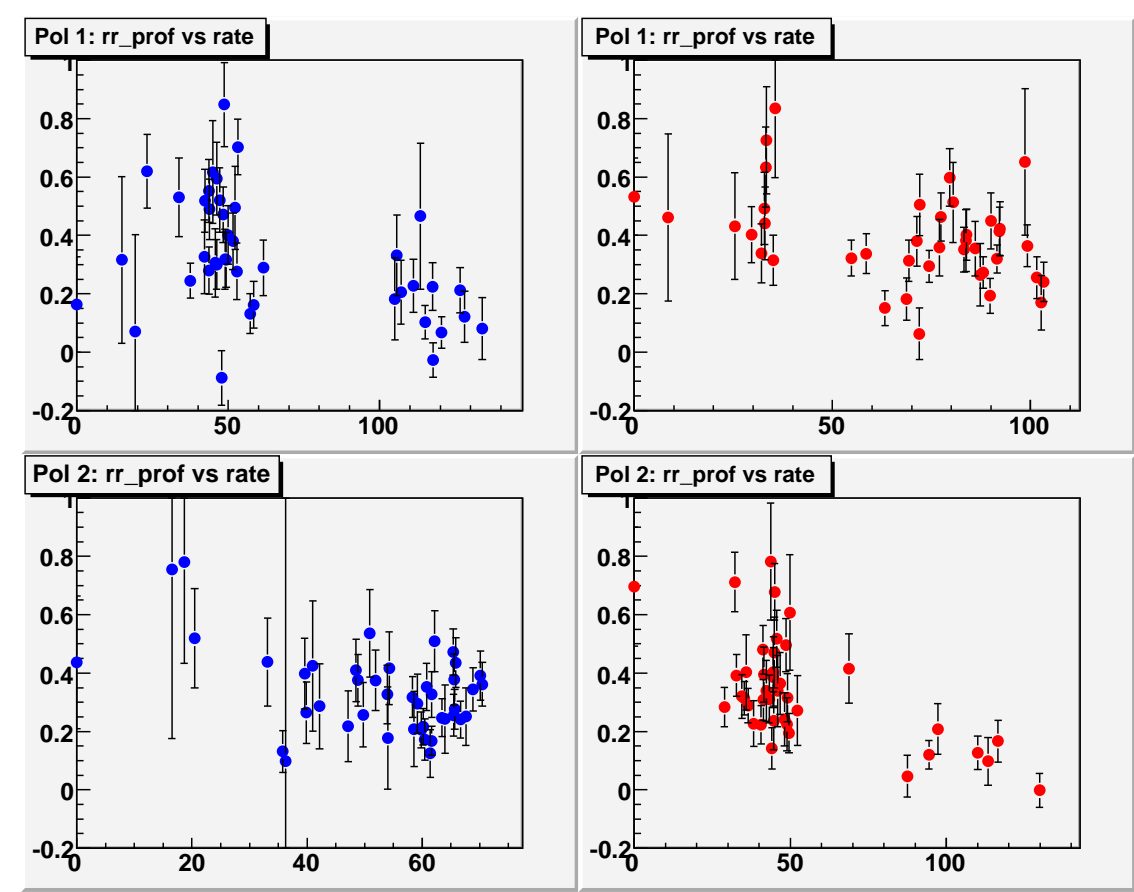

Figure 33. Polarization profile parameter $R$ vs rate per strip $(\mathrm{kHz})$ for $\sqrt{s}=500 \mathrm{GeV}$ data measured by Blue1 (upper-left), Blue2 (bottom-left), Yellow1 (upper-right) and Yellow2 (bottom-right). Data for horizontal and vertical profiles are not separated.
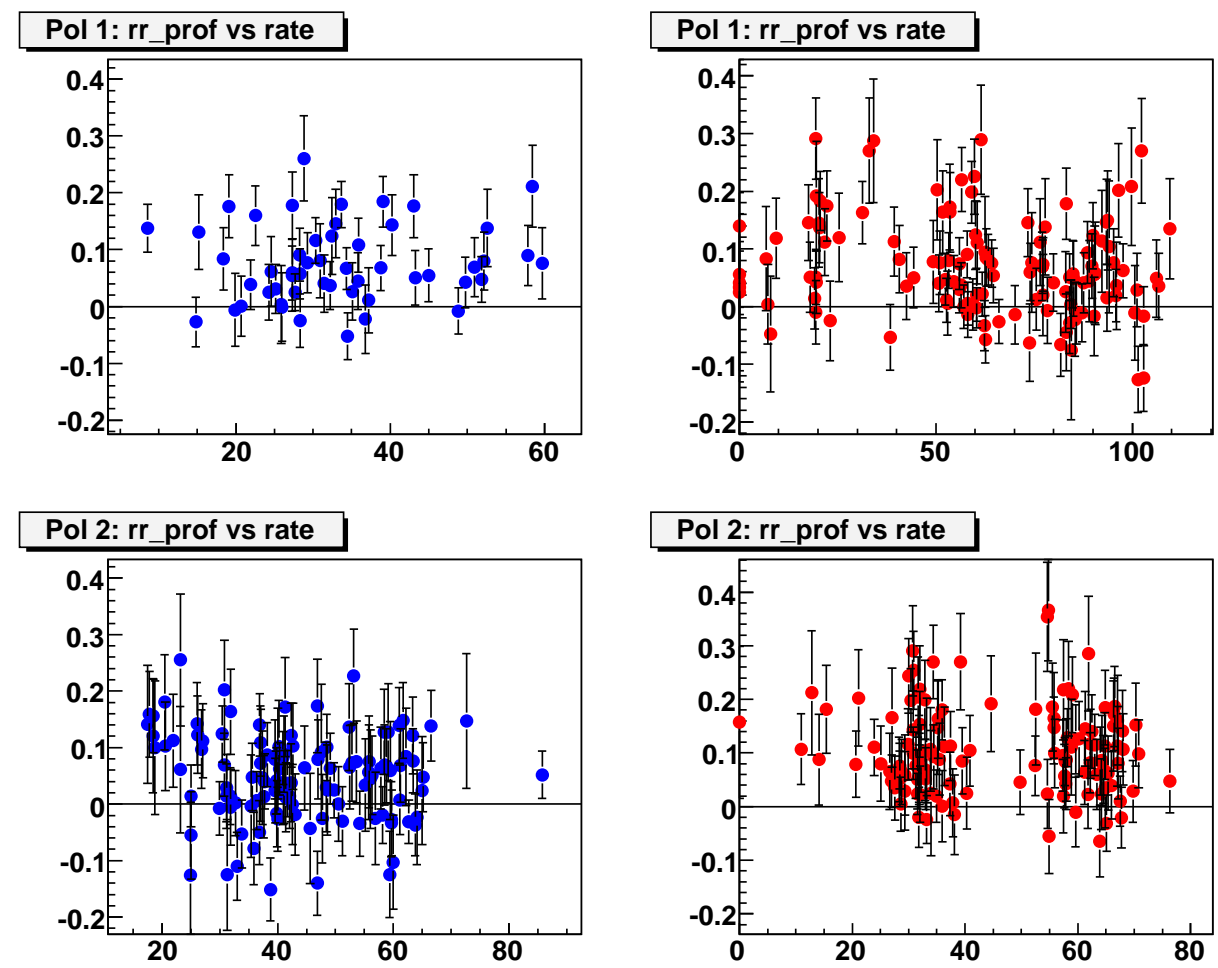

Figure 34. The same as Fig. 33, but for $\sqrt{s}=200 \mathrm{GeV}$ data. 


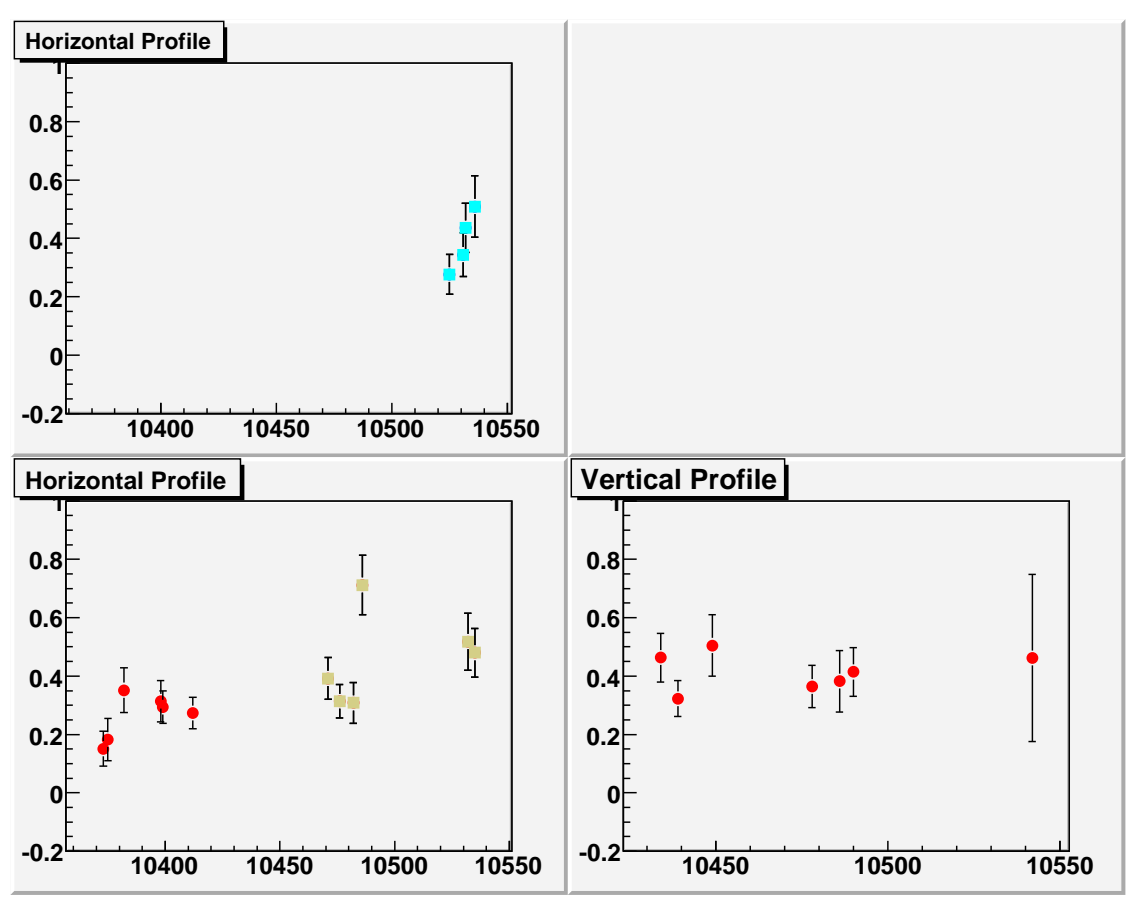

Figure 35. The same as Fig. 31, but only for data with gaussian intensity profiles during $\sqrt{s}=500 \mathrm{GeV}$.
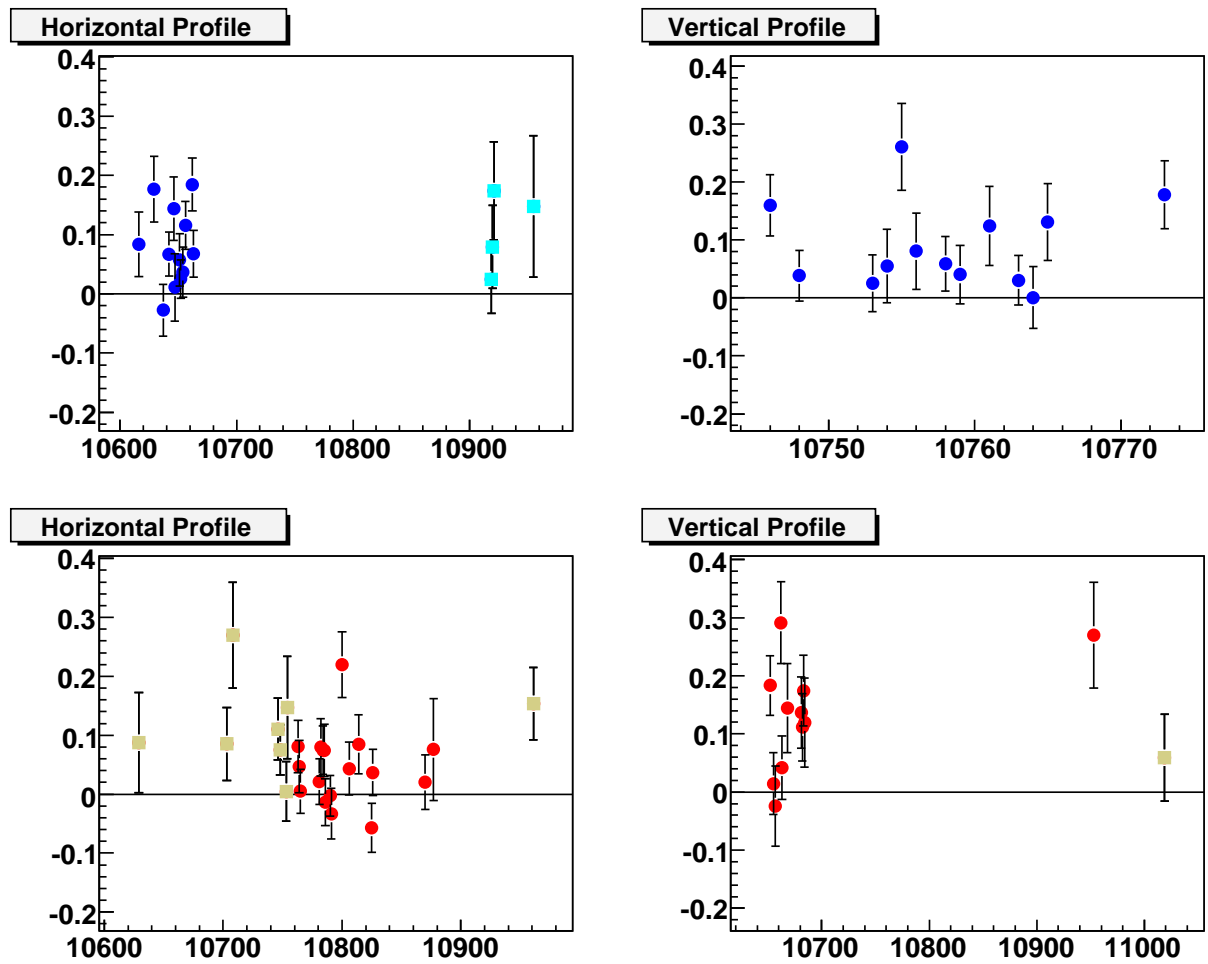

Figure 36. The same as Fig. 32, but only for data with gaussian intensity profiles during $\sqrt{s}=200 \mathrm{GeV}$. 
for $100 \mathrm{GeV}$ beams, similar in both transverse projections. The value of $R$ obtained for $100 \mathrm{GeV}$ beams at RHIC is very similar to the one obtained at RHIC injection and at the AGS [4].

To get a more firm statement about the polarization profiles in Run9 (and to double check the conclusion about the profile made above), we selected only good measurements, when intensity profile was gaussian (not corrupted), as for example the one shown in Fig. 28. Fig. 35 and 36 summarize such measurements in Run9 data, which confirm our conclusion about the average polarization profile parameters $R$ of $\sim 0.4$ for $250 \mathrm{GeV}$ beams and $\sim 0.08$ for $100 \mathrm{GeV}$ beams in Run9. The results are summarized in Table 2 .

Table 2

The polarization profile parameter $R$, average over fills . For the "low rate" case data was used with rates $<30 \mathrm{kHz} /$ strip (notice, only few points satisfied "low rate" condition at $\sqrt{s}=500 \mathrm{GeV}$ (see Fig. 31), so the corresponding numbers can not represent the average $R$ from the whole data sample).

\begin{tabular}{|l|c|c|c|c|}
\hline \hline$\sqrt{s}, \mathrm{GeV}$ & Blue-Horiz & Blue-Vert & Yell-Horiz & Yell-Vert \\
\hline 500, from Fig. 31 (all) & $0.29 \pm 0.02$ & $0.22 \pm 0.02$ & $0.31 \pm 0.02$ & $0.27 \pm 0.02$ \\
500, from Fig. 31 (low rate) & $0.63 \pm 0.09$ & $0.49 \pm 0.10$ & $0.31 \pm 0.07$ & $0.41 \pm 0.08$ \\
500, from Fig. 35 (good prof) & $0.37 \pm 0.04$ & - & $0.32 \pm 0.03$ & $0.39 \pm 0.03$ \\
\hline 200, from Fig. 32 (all) & $0.053 \pm 0.005$ & $0.050 \pm 0.010$ & $0.067 \pm 0.005$ & $0.066 \pm 0.006$ \\
200, from Fig. 32 (low rate) & $0.061 \pm 0.011$ & $0.076 \pm 0.015$ & $0.076 \pm 0.015$ & $0.089 \pm 0.014$ \\
200, from Fig. 36 (good prof) & $0.073 \pm 0.012$ & $0.075 \pm 0.015$ & $0.056 \pm 0.010$ & $0.117 \pm 0.018$ \\
\hline \hline
\end{tabular}

Despite that in Run9 parameter $R$ was supposed to be determined in both transverse directions from scan measurements in each physics fill (by polarimeter- 1 in one projection and by polarimeter- 2 in the other projection), there were only a few fills, which provided reliable measurement of $R$. In the final results we decided to use the same $R$ parameter for all fills (from the average in "good prof" measurements in Table 2), 0.36 for $\sqrt{s}=500$ $\mathrm{GeV}$ and 0.08 for for $\sqrt{s}=200 \mathrm{GeV}$ measurements, with a fill-by-fill uncertainty \pm 0.36 and \pm 0.08 , respectively, which roughly reflects the range of variation of $R$ from fill to fill as from Fig. 31, 32, 35 and 36. The uncertainty on the average of $R$ should enter the global polarization uncertainty (correlated for all fills, separately for $\sqrt{s}=500 \mathrm{GeV}$ and $200 \mathrm{GeV}$ measurements). From the (maximal) variation of the numbers in Table 2 relative to the average values of 0.36 and 0.08 , it was (over)estimated to be \pm 0.14 and \pm 0.04 , for $\sqrt{s}=500 \mathrm{GeV}$ and $200 \mathrm{GeV}$, respectively.

\subsection{Normalization to HJet}

Normaliztion for the $\mathrm{pC}$ measurements is obtained from the comparison of HJet measurements ([5]) with the average beam polarization $\langle P\rangle$ across beam transverse profile obtained by $\mathrm{pC}$. The latter can be taken from a scan measurement $P_{\text {scan }}$, which supposed to measure $\langle P\rangle$. Unfortunately $P_{\text {scan }}$ biases the true value of $\langle P\rangle$ in case we have rate or "loose target" problems. For "loose target" measurements the unbiased value of $\langle P\rangle$ can 
be obtained using Eq. (6). But this approach makes the estimation even worse (compared to just $\left.P_{\text {scan }}\right)$ for the measurements suffering from high rates. Since in our data we can not clearly separate these two effects, we decided to use $P_{\text {scan }}$ for the evaluation of $\langle P\rangle$ by pC. Notice, on the average (over all fills) it doesn't matter what observable from $\mathrm{pC}$ we use for polarization measurements, as long as it is properly normalized to HJet absolute measurements.

Fig. 37, 38, 39, 40 show the comparison of HJet to the $\mathrm{pC}$ measurements as well as their ratio fill by fill for all four $\mathrm{pC}$ polarimeters. All data are consistent with a constant behavior vs fills (within large stat. uncertainites coming mainly from HJet uncertainties).

To make a more precise test, in Fig. 41, 42, 43 and 44 HJet and pC measurements vs periods (luminosity weighted average over fills in a period) are compared, the periods are defined by fills where the target in polarimeter- 1 and polarimeter-2 didn't change (separately for blue and yellow). On this level (on the level of stat. uncertaities) we also do not see any obvious signs of systematic problems in the comparison of HJet and $\mathrm{pC}$ measurements.

The normalization for the $\mathrm{pC}$ measurements was obtained from the comparison of HJet measurements and $\mathrm{pC}$ measurements averaged over the whole run, separetely for each $\mathrm{pC}$ polarimeter and for $\sqrt{s}=200$ and $500 \mathrm{GeV}$. The HJet average polarization over the Run is obtained from the combined statistics over fills (which is fill averaging weighted with number of HJet events, which effectively is luminosity weighted); the $\mathrm{pC}$ average polarization was obtained from the fill polarizations weighted with the number of HJet events in a fill. Results are shown in Table 3.

Table 3

Normalization factor for pC measurements; errors are stat. only.

\begin{tabular}{|c|c|c|c|c|}
\hline \hline$\sqrt{s}, \mathrm{GeV}$ & Blue1 & Blue2 & Yell1 & Yell2 \\
\hline 500 & $0.849 \pm 0.021$ & $0.839 \pm 0.021$ & $0.885 \pm 0.024$ & $0.853 \pm 0.023$ \\
200 & $0.976 \pm 0.015$ & $0.956 \pm 0.009$ & $0.992 \pm 0.010$ & $0.989 \pm 0.010$ \\
\hline \hline
\end{tabular}

\subsection{Polarization decay in a fill}

Since we do not have a clear view how to correct the rate effect (and it was corrected only partially by "Dead Layer" apporach), it is hard to derive any precise statement about polarization decay in a fill from Run9 data. Rate effects tend to lead to lower measured asymmetries, so that the polarization decay time extracted from data will underestimate the actual polarization decay time (due to that event rate decreases from the beginning to the end of a fill, which would bias the measured polarizations to higher values at the end of a fill compared to the beginning of the fill).

Fig. 45 and 46 show the fill by fill $1 / t_{\text {decay }}$ extracted from an exp fit to the polarization vs time of the measurements (see for example Fig. 47).

At $\sqrt{s}=200 \mathrm{GeV}$ the Blue beam on the average showed $1 / t_{\text {decay }}$ around 0.005 , which corresponds to $t_{\text {decay }} \sim 200$ hours. The Yellow beam showed a similar decay time, which looks decreased in the end of Run9 to $t_{\text {decay }} \sim 50$ hours. For the comparison, $t_{\text {decay }}$ 


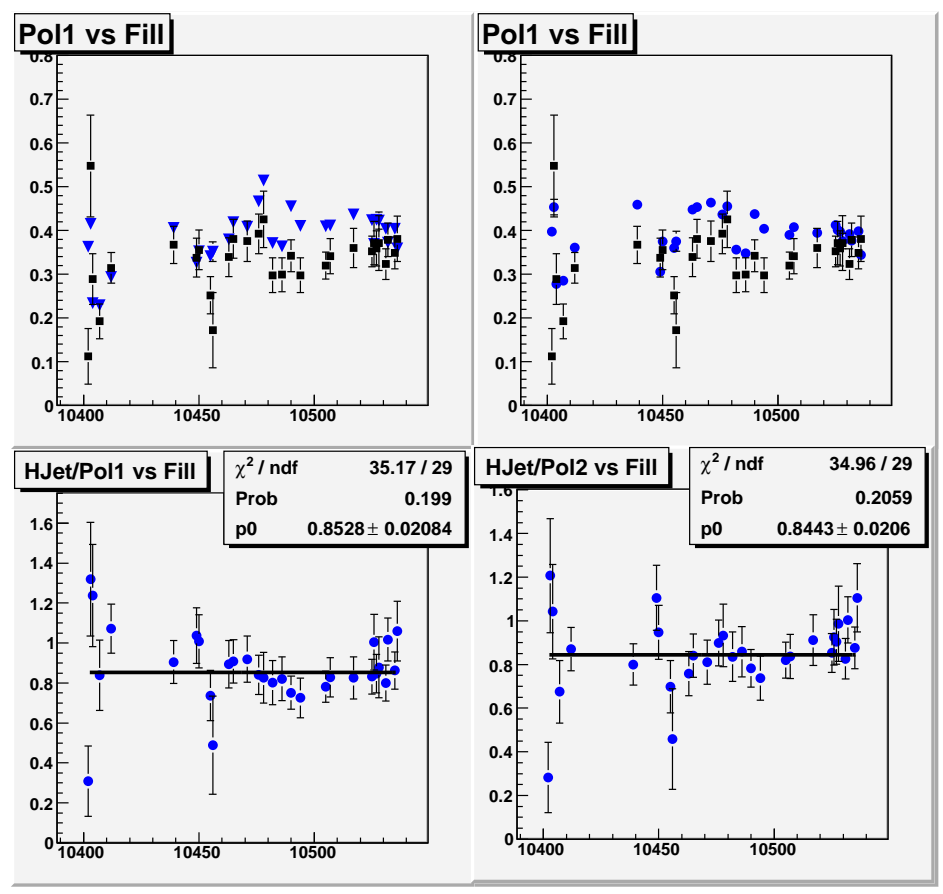

Figure 37. Upper plots: comparison of fill-by-fill polarizations measured by HJet (black squares) and $\mathrm{pC}$ in blue ring at $\sqrt{s}=500 \mathrm{GeV}$, left for Blue1, right for Blue2; Bottom plots: ratio of HJet over pC polarizations, fill by fill, left for Blue1, right for Blue2.

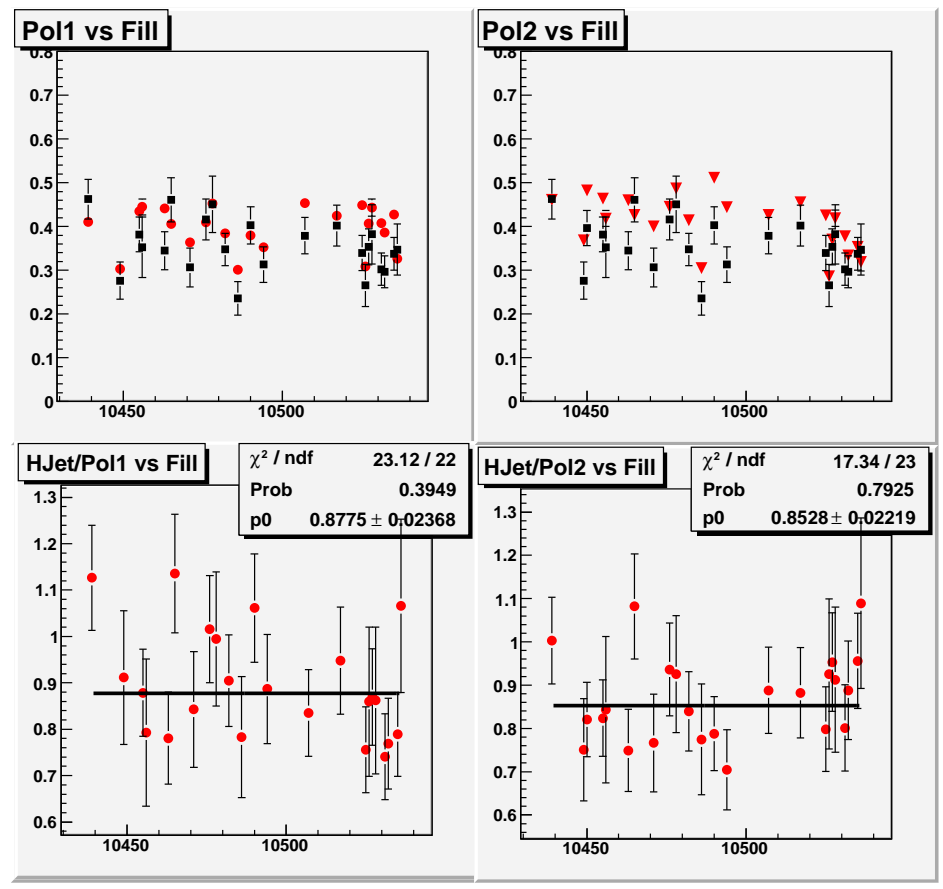

Figure 38. The same as Fig. 37, but for yellow ring at $\sqrt{s}=500 \mathrm{GeV}$. 

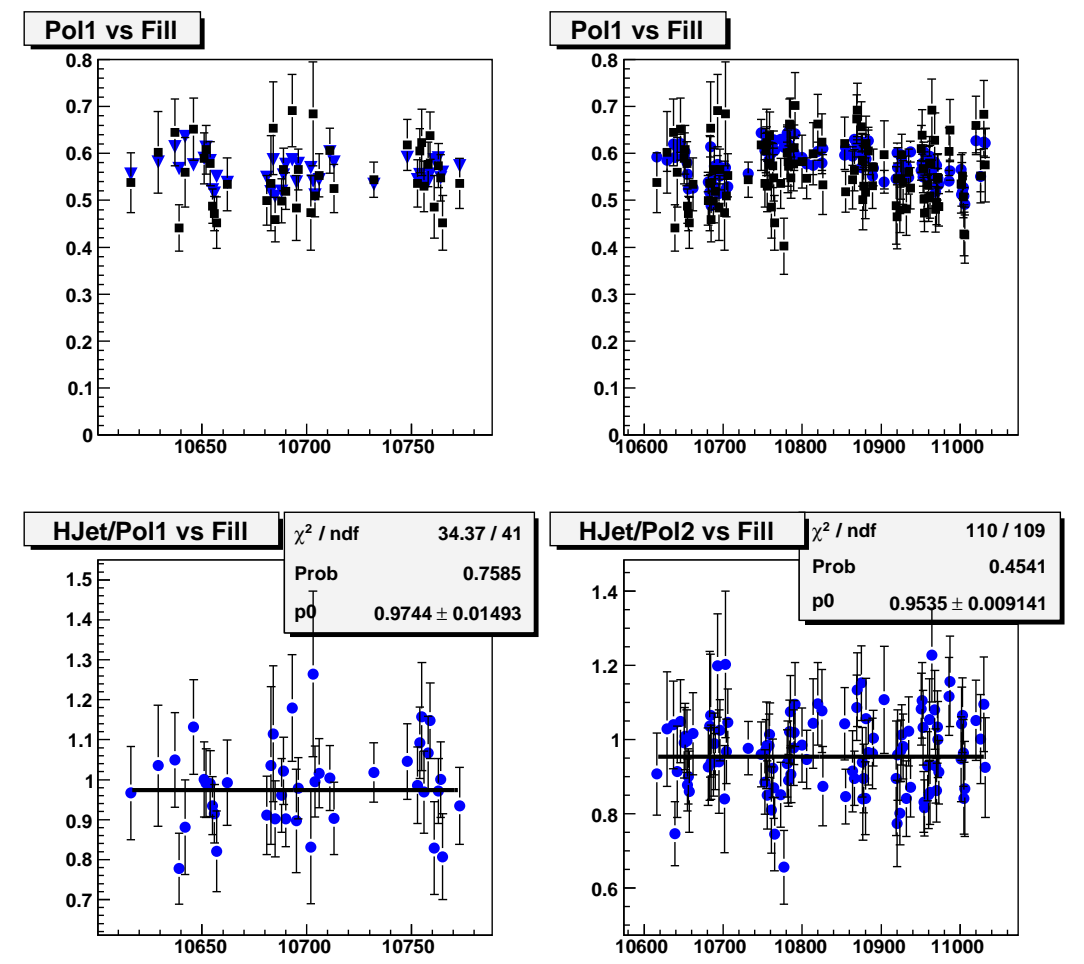

Figure 39. The same as Fig. 37, but for blue ring at $\sqrt{s}=200 \mathrm{GeV}$.
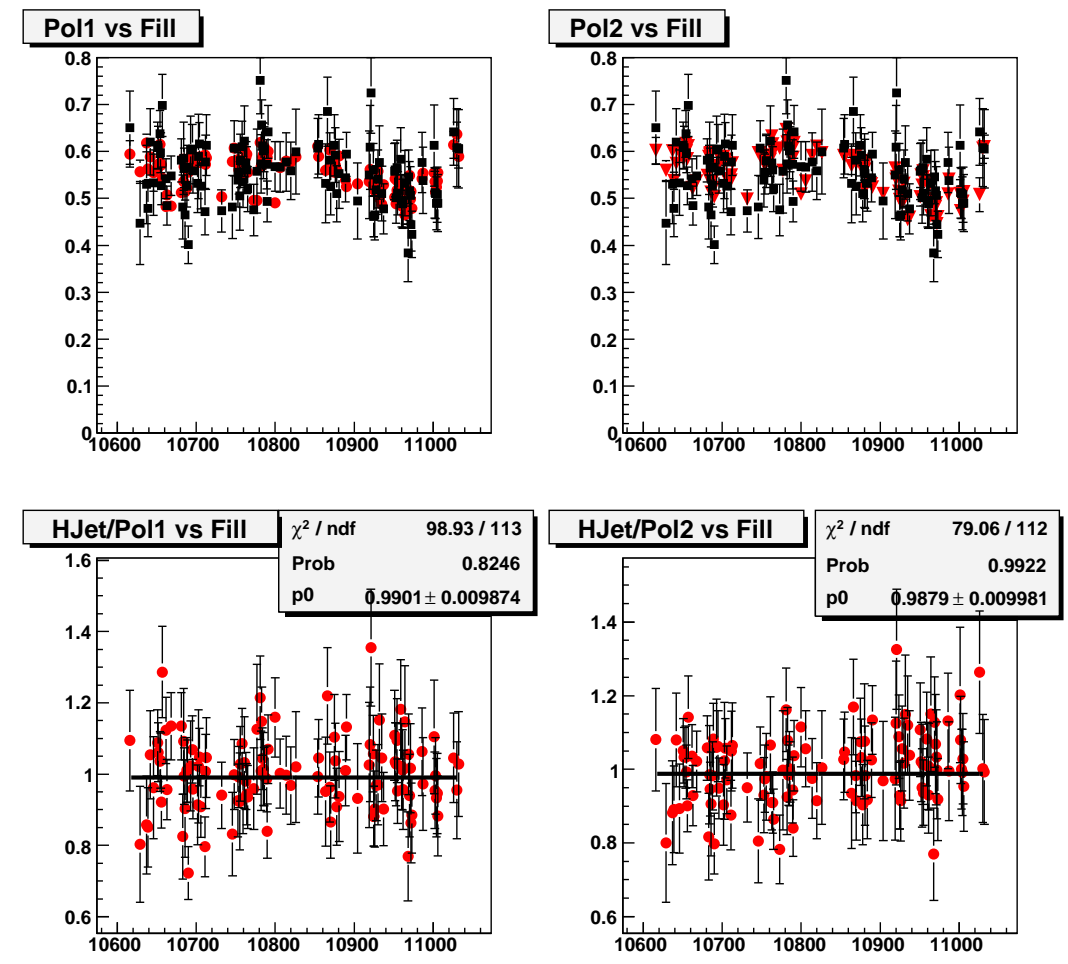

Figure 40. The same as Fig. 37, but for yellow ring at $\sqrt{s}=200 \mathrm{GeV}$. 


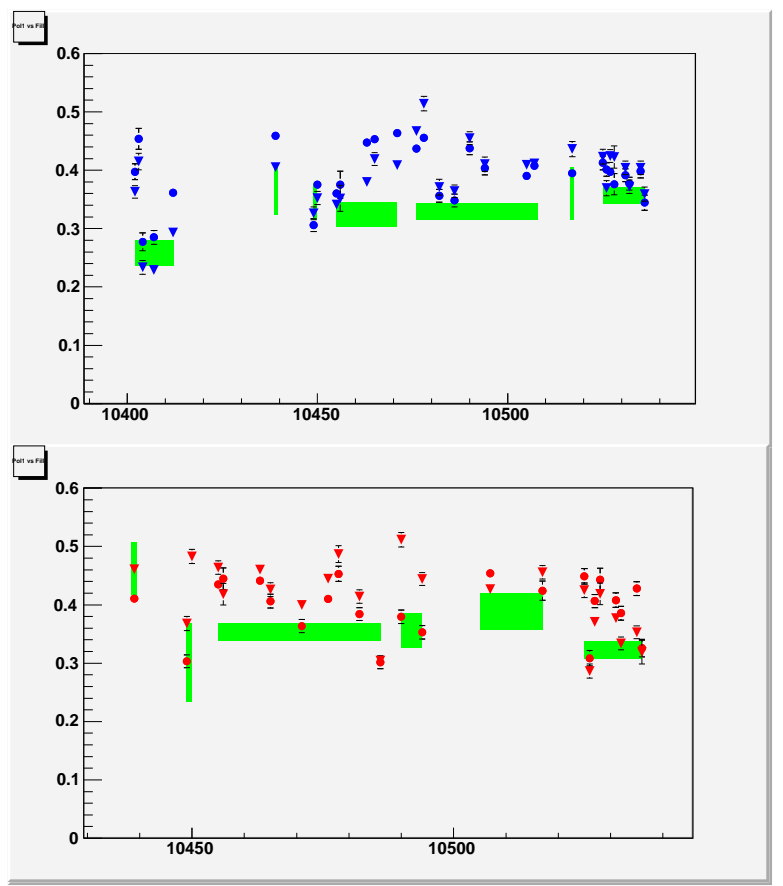

Figure 41. Comparison of fill-by-fill pC polarization measurements (triangles for polarimeter-1 and circles for polarimeter-2) with HJet measurements averaged over groups of fills denoted by horizontal length of the green bars, vertical size of the bars being $\pm 1 \sigma$ stat. uncertainty for a period; data for $\sqrt{s}=500 \mathrm{GeV}$ blue (upper) and yellow (bottom). $\mathrm{pC}$ measurements are shown only for fills, which also have HJet values.

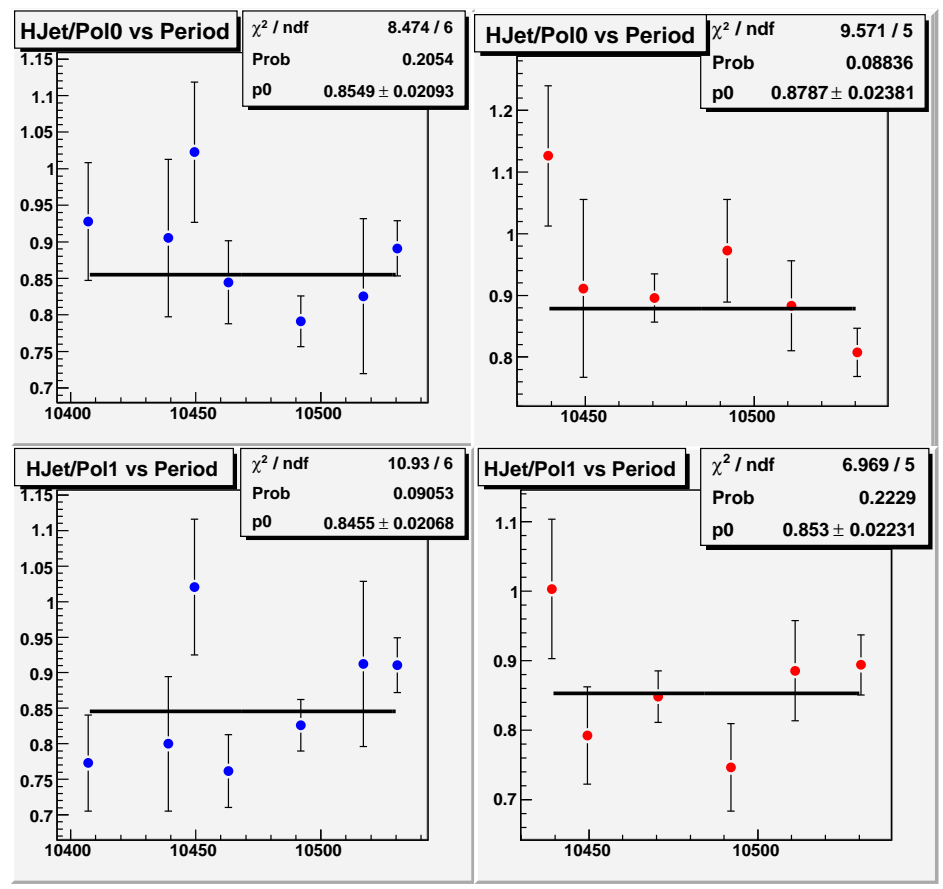

Figure 42. Ratio of the HJet polarization measurements over pC measurements, periodby-period; periods are defined by a group of fills where the target in polarimeter- 1 and polarimeter-2 didn't change (separately for blue and yellow); upper left for Blue1, bottom left for Blue2, upper right for Yellow1, bottom right for Yellow2. 

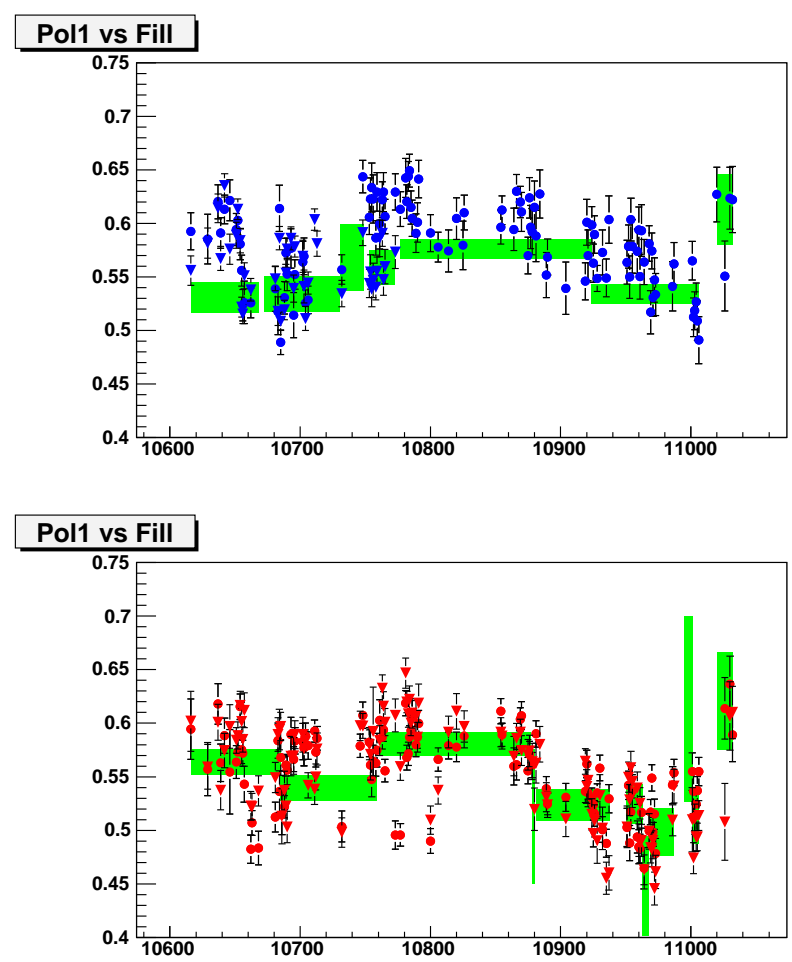

Figure 43. The same as Fig. 41, but for $\sqrt{s}=200 \mathrm{GeV}$.
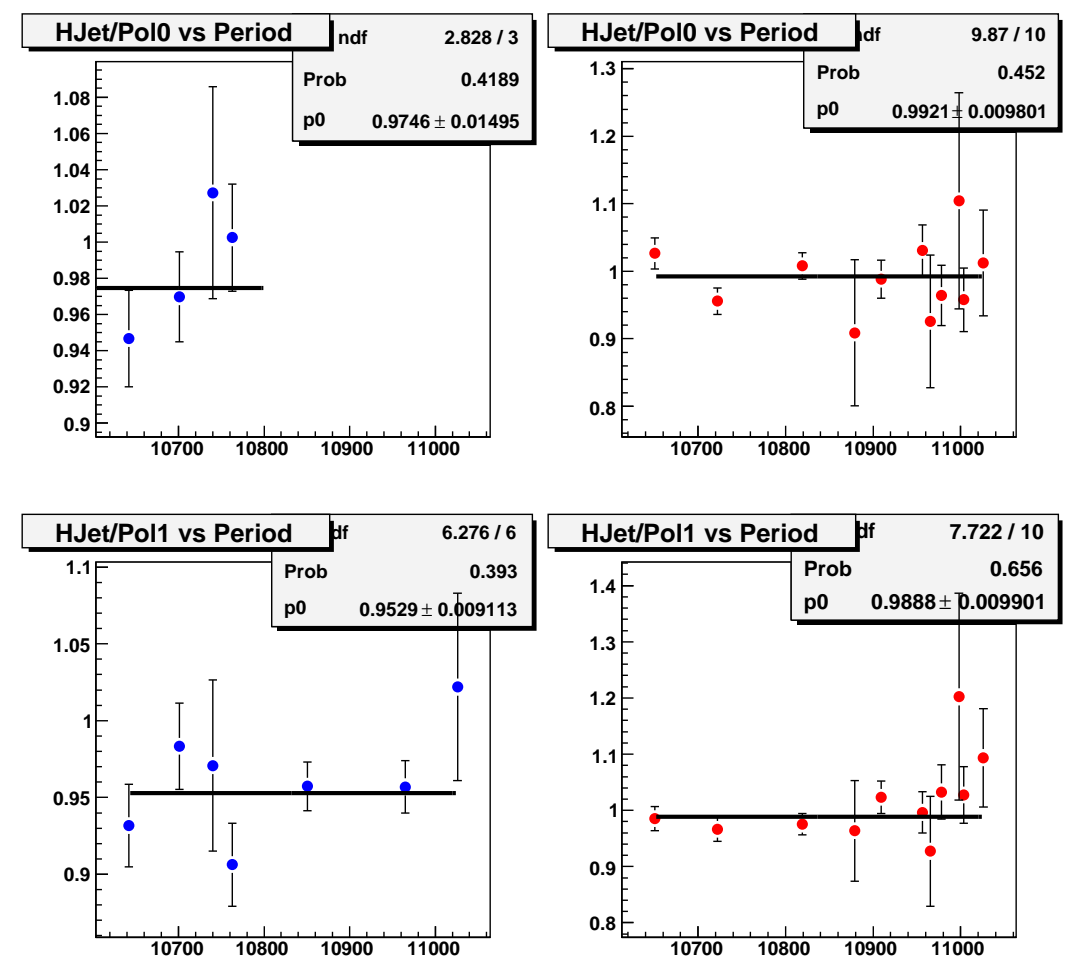

Figure 44. The same as Fig. 42 , but for $\sqrt{s}=200 \mathrm{GeV}$. 
measured in Run6 (Run8) were $~ 150$ hours (100-400 hours). A few fills clearly showed shorter polarization decay time, consistently in Polarimeter-1 and Polarimeter-2. For example fills 10773 and 10777 in yellow with $t_{\text {decay }} \sim 20$ hours (see for example Fig. 47).

$\sqrt{s}=500 \mathrm{GeV}$ data was more affected by high rates, so the $t_{\text {decay }}$ measurements in this data sample are expected to be more biased towards longer $t_{\text {decay }}$ values. From Fig. 45 we can obtain $t_{\text {decay }} \sim 50$ hours for fills $>10450$ and varying in a wide range $(\sim 10-100$ hours $)$ for fills $<10450$. One of the extreme cases with $t_{\text {decay }} \sim 6$ hours in blue ring is shown in Fig. 48). All measurements in yellow ring showed $t_{\text {decay }}>30$ hours.

For the polarization average over a fill we use the weighted average of all measurements in a fill, which due to polarization and luminosity decay vs time may not precisely reflect the real polarization average over a fill. To estimate the possible effect, in Fig. 49 and 50 we compare the measurements of the average polarization obtained from the fit to a constant and from the averaging taking into account the polarization decay with the slopes as determined in Fig. 45 and 46, and the luminosity decay with $t_{\text {decay }}=10$ hours (for $\sqrt{s}=500 \mathrm{GeV}$ ) and 5 hours (for $\sqrt{s}=200 \mathrm{GeV}$ ); these are extreme cases (the lower bound of observed luminosity decay): the smaller $t_{\text {decay }}$ the larger possible discrepancy. The deviation of the ratio from one in each fill in Fig. 49 and 50 can serve as a possible systematic uncertainty for averaging the polarization in a fill. Except for a few fills (usually with sharper polarization decay), data do not show any indication of a sizable syst. uncertainty. We assigned this additional syst. uncertainty for the final results for fills, which show a deviation by more than stat. uncertainty. Only a few fills have a the syst. uncertainty comparable to other fill-by-fill uncertainties listed in Table 4: 10685 in Blue, 10777 and 10963 in yellow for the $\sqrt{s}=200 \mathrm{GeV}$ sample (deviation $>5 \%$ ); and 10375 and 10398 in Blue for the $\sqrt{s}=500 \mathrm{GeV}$ sample (deviation > 8\%).

\subsection{Spin direction in pC (up-down vs left-right asymmetries)}

Fig. 51 and 52 show the $\tan (\phi)$ vs fill, where $\phi$ is the angle between the proton spin direction and vertical axis (calculated from the ratio of up-down over left-right asymmetries).

$200 \mathrm{GeV}$ data consistently show non-vertical spin direction with $\phi \sim 0.15-0.20(8-12$ degrees) for blue beam and $\phi \sim 0.1$ ( 6 degrees) for yellow beam, which would introduce a correction for the measured polarization (if extracted from left-right asymmetry) of 1-2\% and $0.5 \%$ for blue and yellow beams, respectively.

$500 \mathrm{GeV}$ data is less precise due to lower beam polarization and the rate effects, but all data points appear to be in the $\phi$ range $[-0.1,0.1]$, which may give a correction for the measured polarization of no more than $\pm 0.5 \%$.

\subsection{Final polarizations and uncertainties}

Since we do not clearly see the preference of using one polarimeter over the other, the final polarization in each fill is calculated from the weighted average between measurements by Polarimeter- 1 and Polarimeter-2, after they are normalized using the normalization factors from Table 3. For the weights we used $1 / \sigma^{2}$, where $\sigma$ is a squared sum of stat. and syst. uncertainties due to averaging introduced in Section 6.4. To provide polarization values for the experiments (in a collision), these fill by fill polarization values 


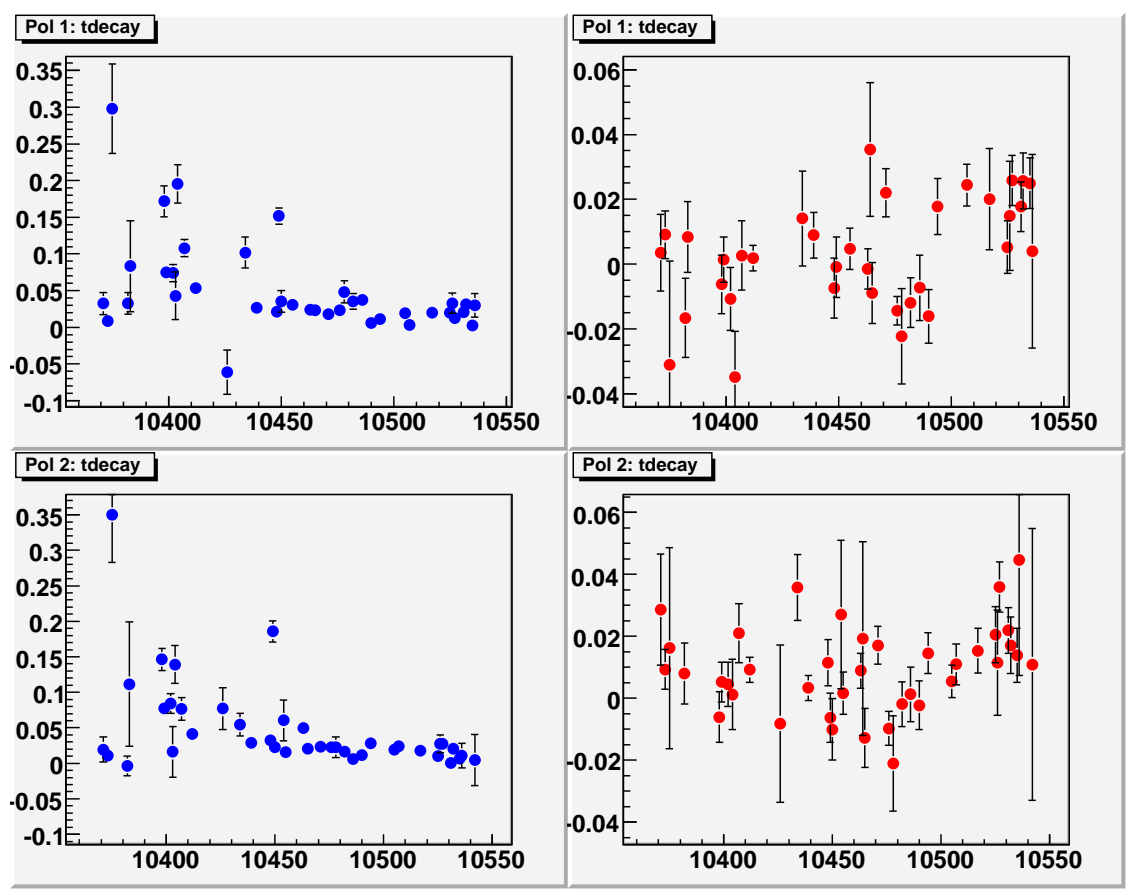

Figure 45. 1/t decay $(1 /$ hours) vs fill; top left - Blue1, bottom left - Blue2, top right Yellow1 and bottom right - Yellow2.
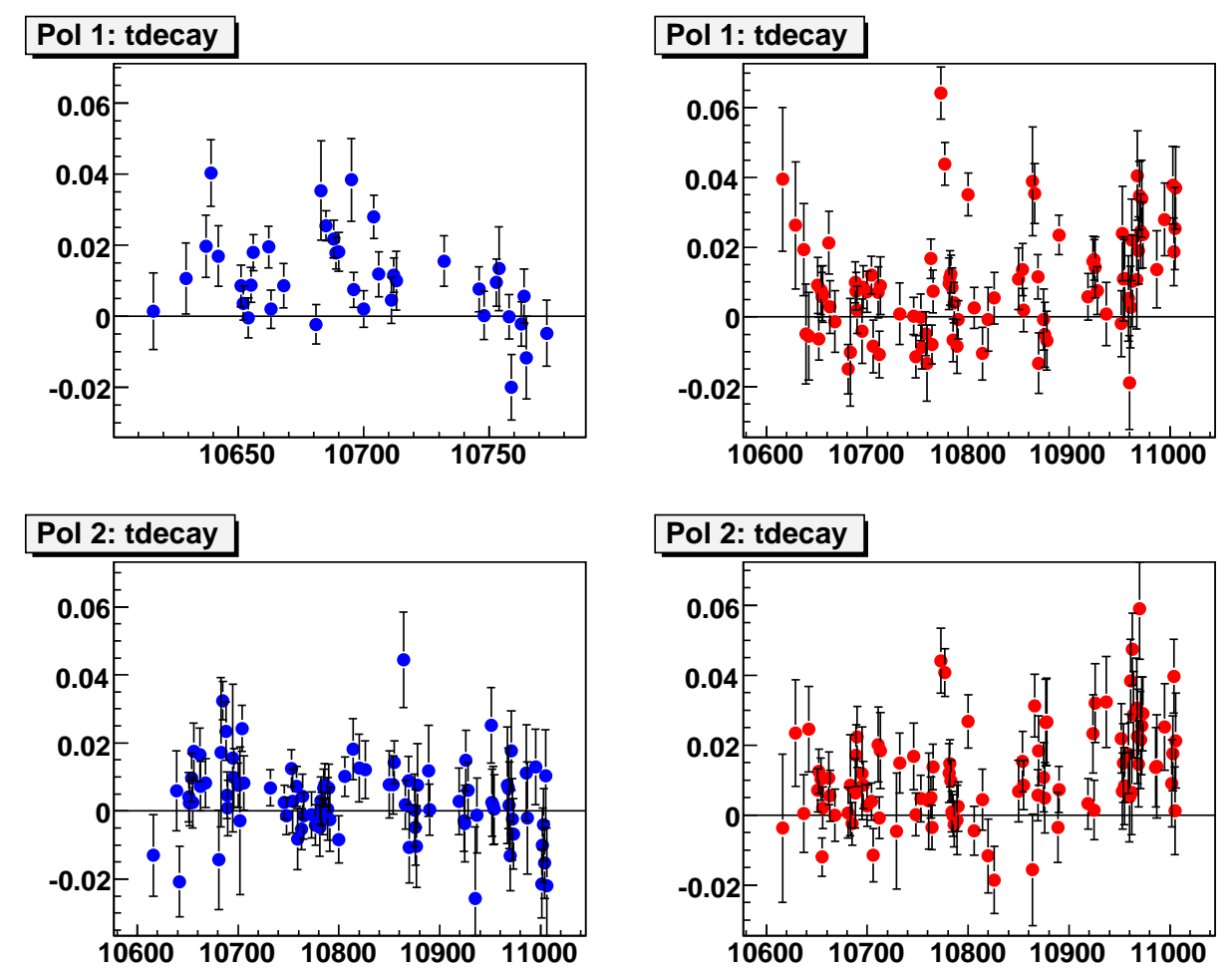

Figure 46. The same as Fig. 45 , but for $\sqrt{s}=200 \mathrm{GeV}$ measurements; notice that Blue1 didn't have measurements for fills $>10773$. 

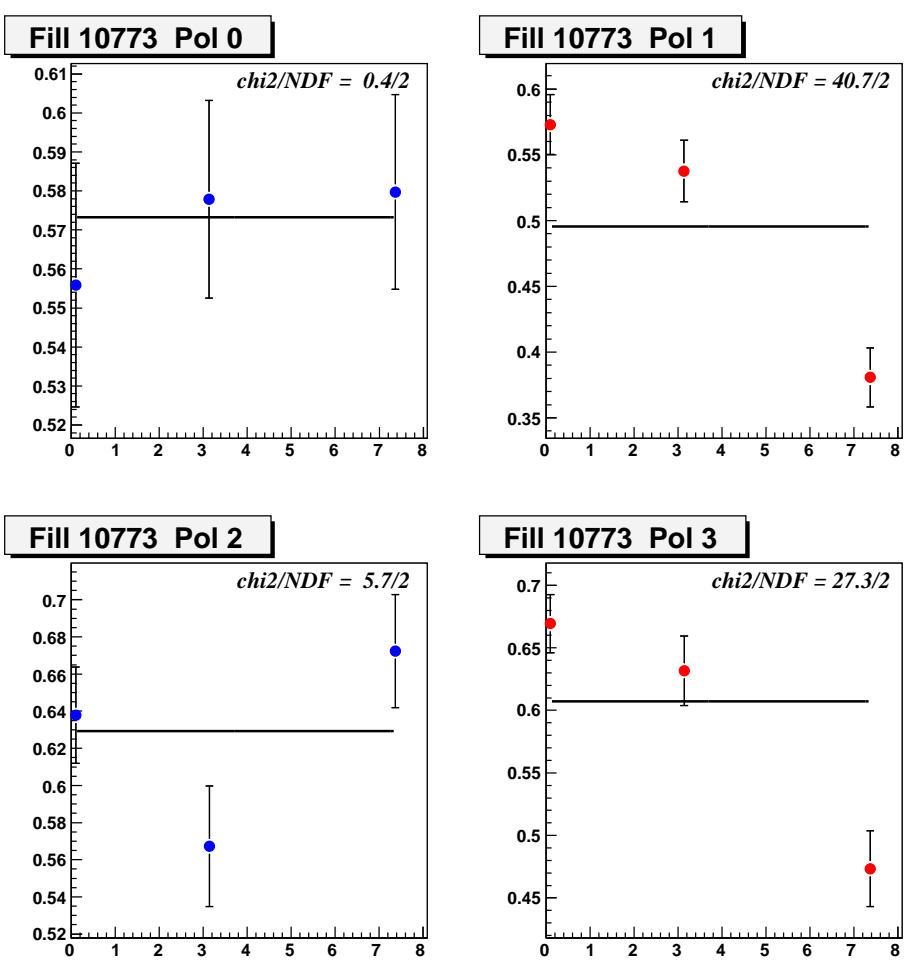

Figure 47. Polarization vs time (hours) for Fill $10773(\sqrt{s}=200 \mathrm{GeV})$ measured by Blue1 (top left), Blue2 (bottom left), Yellow1 (top right) and Yellow2 (bottom right); Line is a fit to a constant.
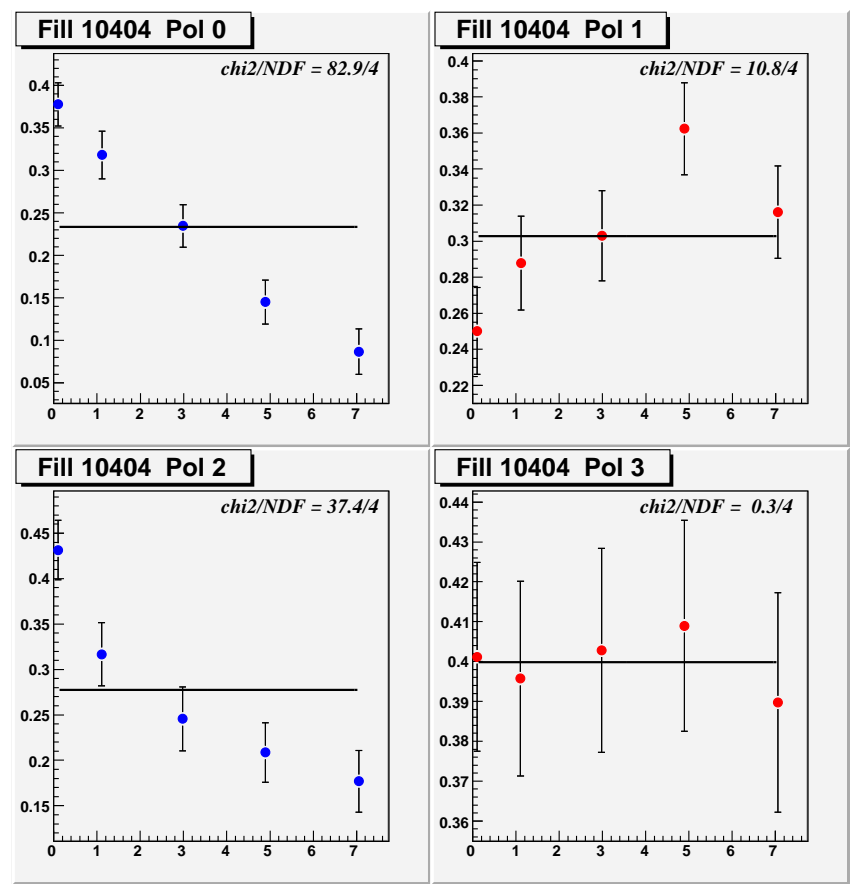

Figure 48. The same as Fig. 47 but for fill $10404(\sqrt{s}=500 \mathrm{GeV})$. 


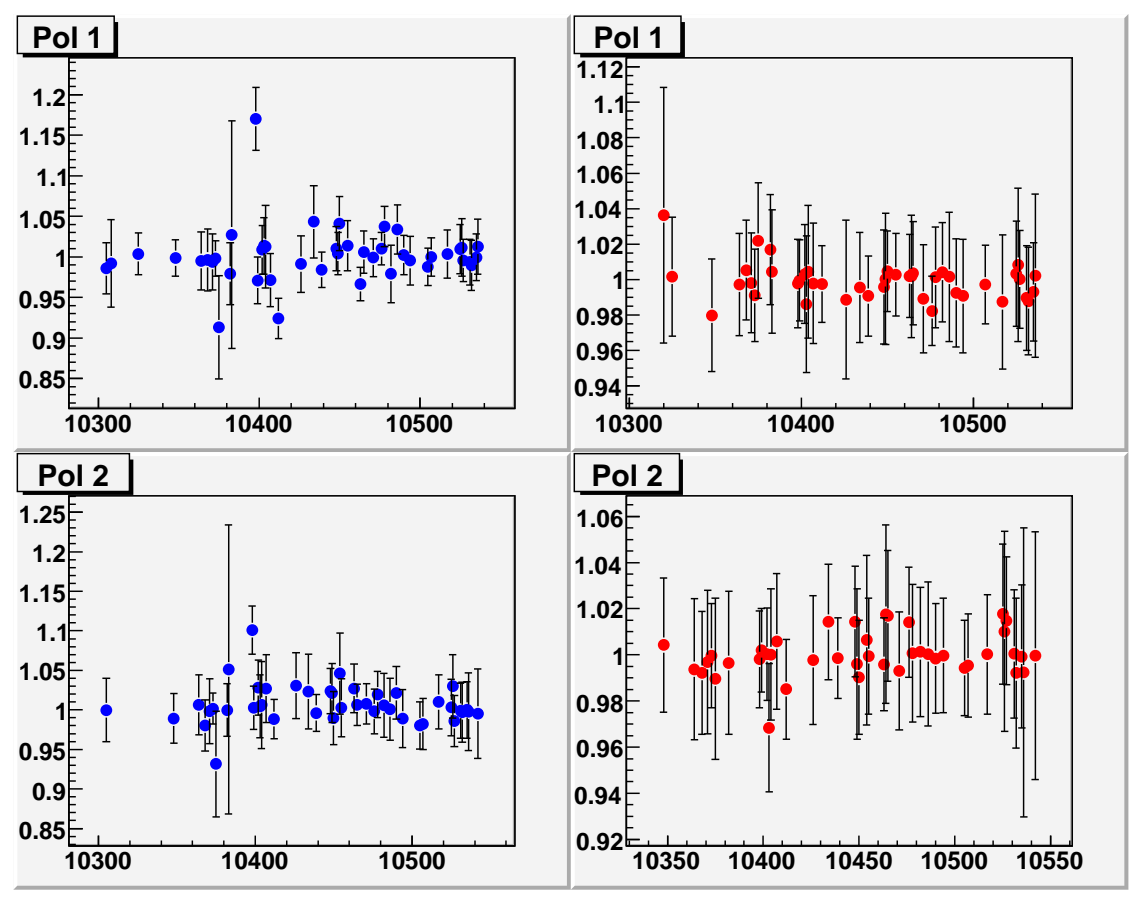

Figure 49. Ratio of the average polarization obtained from weighted average of measurements in a fill (equivalent to a fit to a constant) and from the averaging taking into account $\exp$ decay of polarization (Fig. 45 and 46) and luminosity with $t_{\text {decay }}=10$ hours (extreme case); error bars are stat. uncertainties of the weighted average measurement; top left - Blue1, bottom left - Blue2, top right - Yellow1 and bottom right - Yellow2.
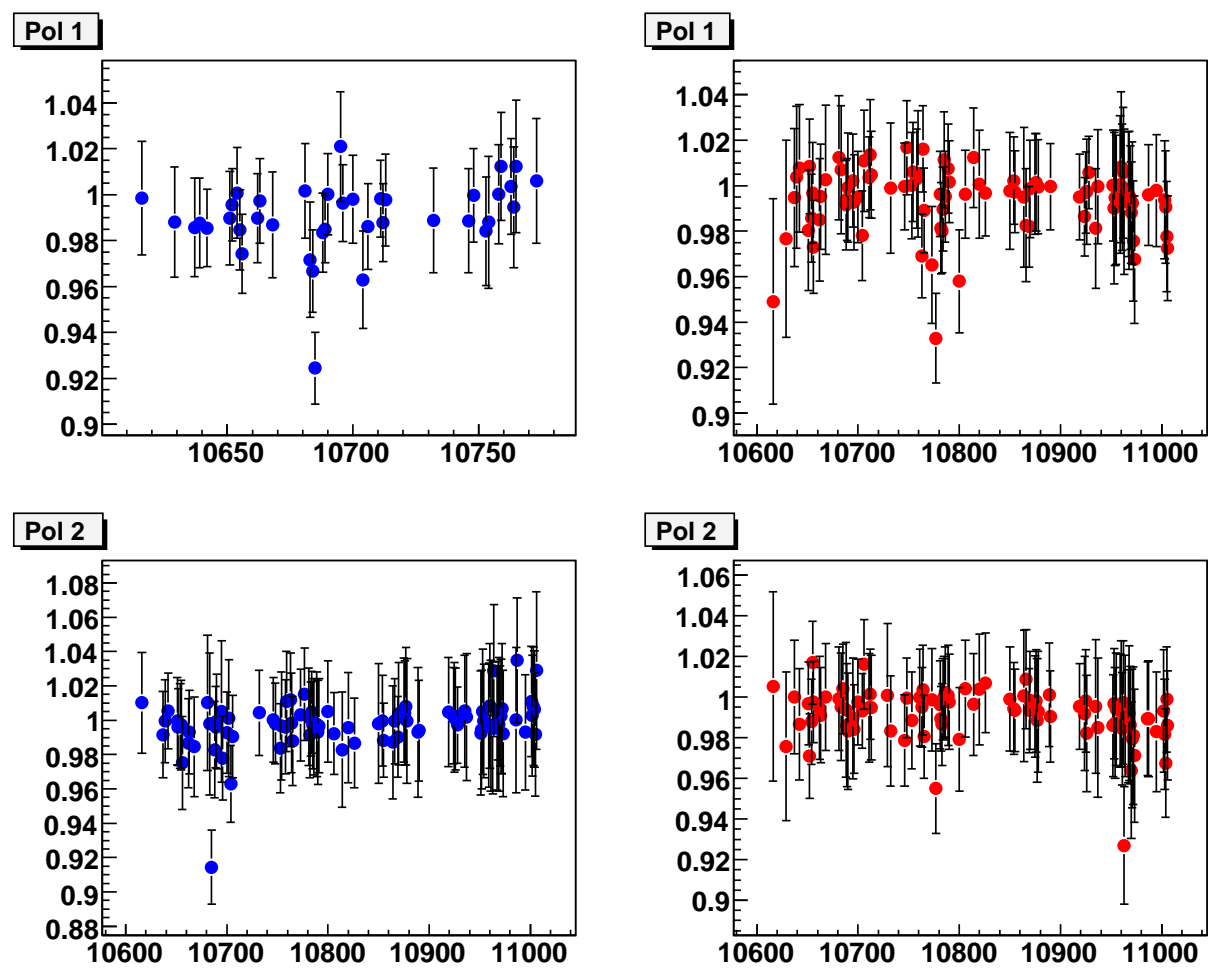

Figure 50. The same as Fig. 49 , but for $\sqrt{s}=200 \mathrm{GeV}$ measurements; luminosity $t_{\text {decay }}=5$ hours is used; notice that Blue1 didn't have measurements for fills $>10773$. 

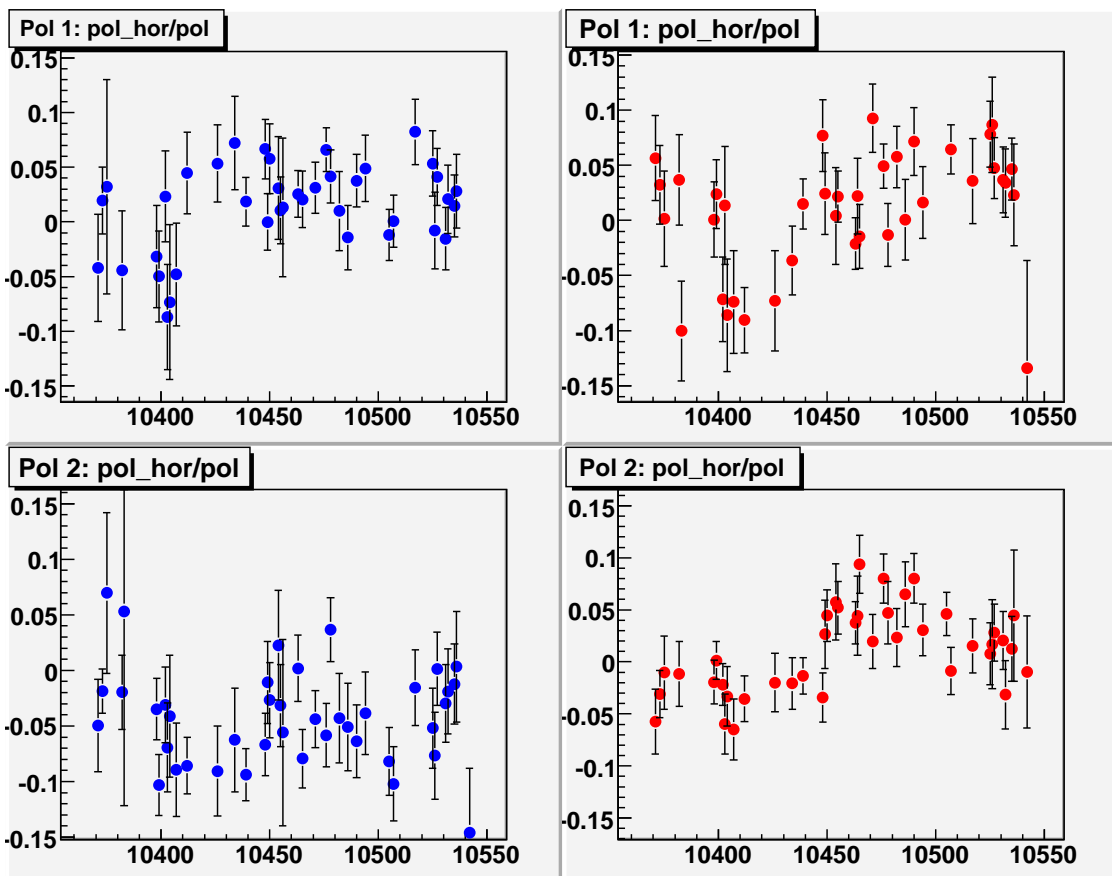

Figure 51. $\tan (\phi)$ vs fill, where $\phi$ is the angle between proton spin direction and the vertical axis (calculated from the ratio of up-down over left-right asymmetries); top left Blue1, bottom left - Blue2, top right - Yellow1 and bottom right - Yellow2.
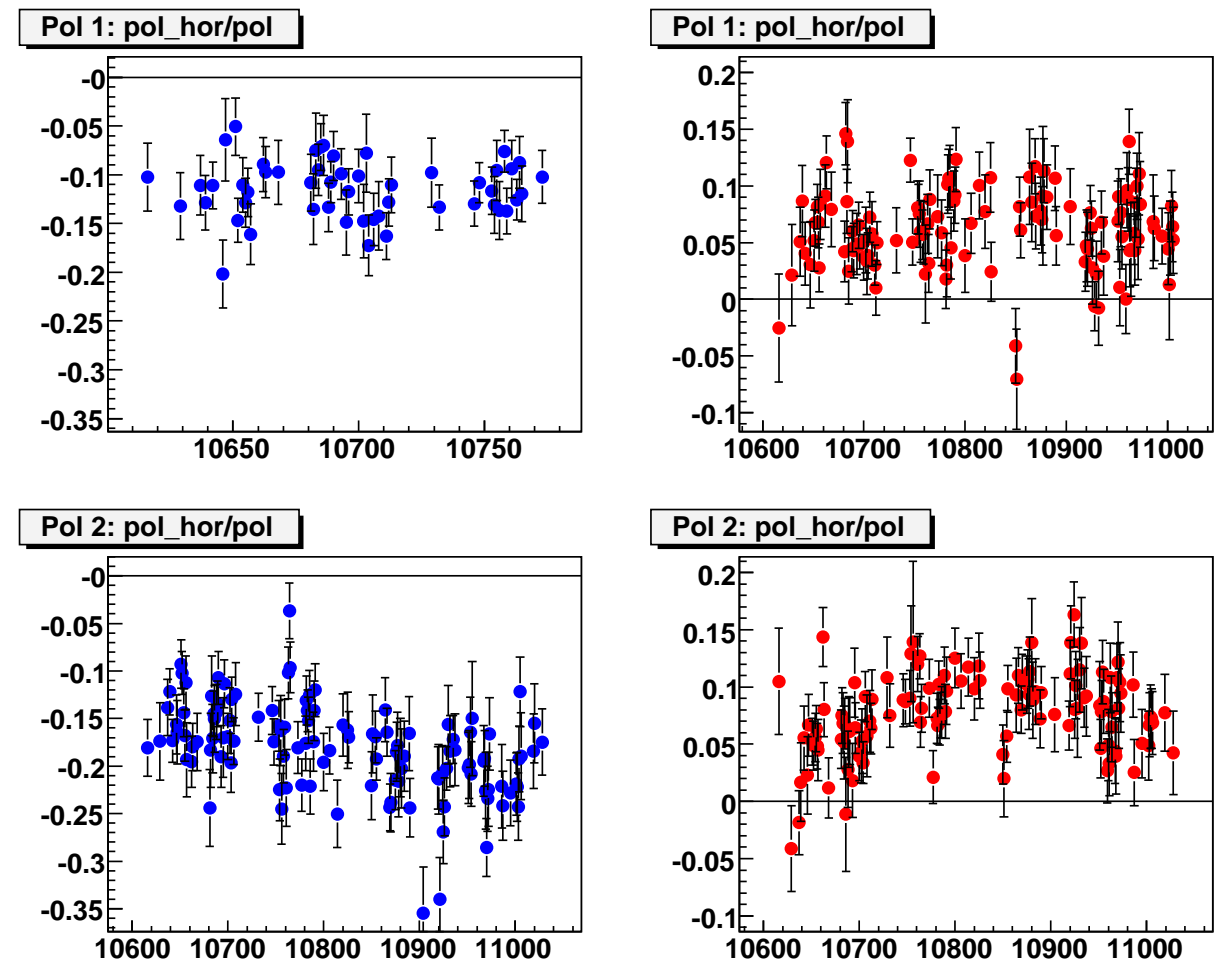

Figure 52. The same as Fig. 51 , but for $\sqrt{s}=200 \mathrm{GeV}$ measurements; notice that Blue1 didn't have measurements for fills $>10773$. 
were corrected for the polarization profile by a factor

$$
k_{e x p}=\frac{\sqrt{\left(1+R_{X}\right) \cdot\left(1+R_{Y}\right)}}{\sqrt{\left(1+\frac{1}{2} R_{X}\right) \cdot\left(1+\frac{1}{2} R_{Y}\right)}},
$$

obtained from the ratio of right-side of Eq. (4) and (2).

According to the discussion in Section 6.2 , we use $R_{X}=R_{Y}=0.36$ and 0.08 , which lead to $k_{\text {exp }}=1.15$ and 1.04 for $\sqrt{s}=500 \mathrm{GeV}$ and $200 \mathrm{GeV}$, respectively.

Fill by fill results obtained in this way (corrected for pol. profile and normalized to HJet) are show in Fig. 53 and 54.

Table 4 and 5 summarize the uncertainties for polarization measurements in Run9.

Table 4

Fill-by-fill (non-correlated) systematic uncertainties. "Pol1 vs Pol2" relates to the inconsistencies in measurements between Polarimeter-1 and Polarimeter-2 discussed in Section 6.1; "Pol. profile" relates to possible variation of the pol. profile (and uncertainty in its definition on fill level) discussed in Section 6.2 (variations in two trans. directions considered as correlated); not included in the table are uncertainties due to averaging discussed in Section 6.1 and shown in Fig. 49 and 50.

\begin{tabular}{|l|c|c|c|c|}
\hline \hline & $\begin{array}{c}\text { Blue } \\
500 \mathrm{GeV}\end{array}$ & $\begin{array}{c}\text { Yellow } \\
500 \mathrm{GeV}\end{array}$ & $\begin{array}{c}\text { Blue } \\
200 \mathrm{GeV}\end{array}$ & $\begin{array}{c}\text { Yellow } \\
200 \mathrm{GeV}\end{array}$ \\
\hline Pol1 vs Pol2 & $12 \%$ & $12 \%$ & $6 \%$ & $6 \%$ \\
Pol. profile & $15 \%$ & $15 \%$ & $4 \%$ & $4 \%$ \\
\hline Total & $19.2 \%$ & $19.2 \%$ & $7.2 \%$ & $7.2 \%$ \\
\hline \hline
\end{tabular}

The "Time dependent inconsistency" is derived from the "global" inconsistency between Polarimeter-1 and Polarimeter-2 measurements in Fig. 26 and 27. This uncertainty won't be present in physics results in case only fills, which have a HJet measurement are used in the physics analysis (and used with full collected luminosity). So this uncertainty roughly accounts for fills for which we do not have absolute polarization measurements by HJet (about half of all fills in $\sqrt{s}=500 \mathrm{GeV}$ sample and about a quarter in $\sqrt{s}=200 \mathrm{GeV}$ sample); and for the case if one uses only a subsample of the total data sample, which may show some systematic variations relative to average line as seen from Fig. 26 and 27.

For the double spin asymmetry measurements a product of two beam polarizations $P_{B} \cdot P_{Y}$ is used and the global uncertainty $\delta\left(P_{B} \cdot P_{Y}\right) /\left(P_{B} \cdot P_{Y}\right)$ can be derived from Table 5 considering that "Jet normalization, syst" as well as "Time dependence" uncertainties are mostly correlated between blue and yellow beams (to insure that we do not underestimate the total uncertainties). They are $18.5 \%$ for $500 \mathrm{GeV}$ :

$$
\sqrt{2.5^{2}+2.7^{2}+(2.0+2.0)^{2}+(3.0+3.0)^{2}+(5.0+10.0)^{2}+5.0^{2}+5.0^{2}} ;
$$

and $8.8 \%$ for $200 \mathrm{GeV}$ :

$\sqrt{1.0^{2}+1.0^{2}+(2.0+2.0)^{2}+(2.0+2.0)^{2}+(3.0+3.0)^{2}+2.0^{2}+2.0^{2}}$.

Calculated in the similar way the relative uncertainty for the average between two beam polarization $\left(P_{B}+P_{Y}\right) / 2$ (used in single spin asymmetry measurements, when data from 

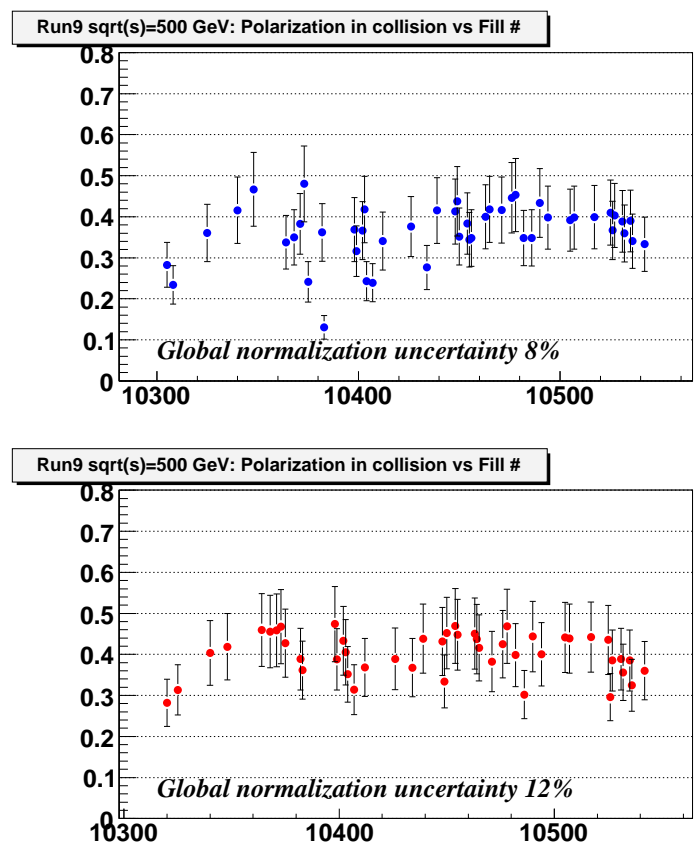

Figure 53. Fill-by-fill polarizations in collisions for Blue beam at $\sqrt{s}=500 \mathrm{GeV}$, corrected for pol. profile and normalized to HJet; uncertainties are stat. and fill-by-fill syst.

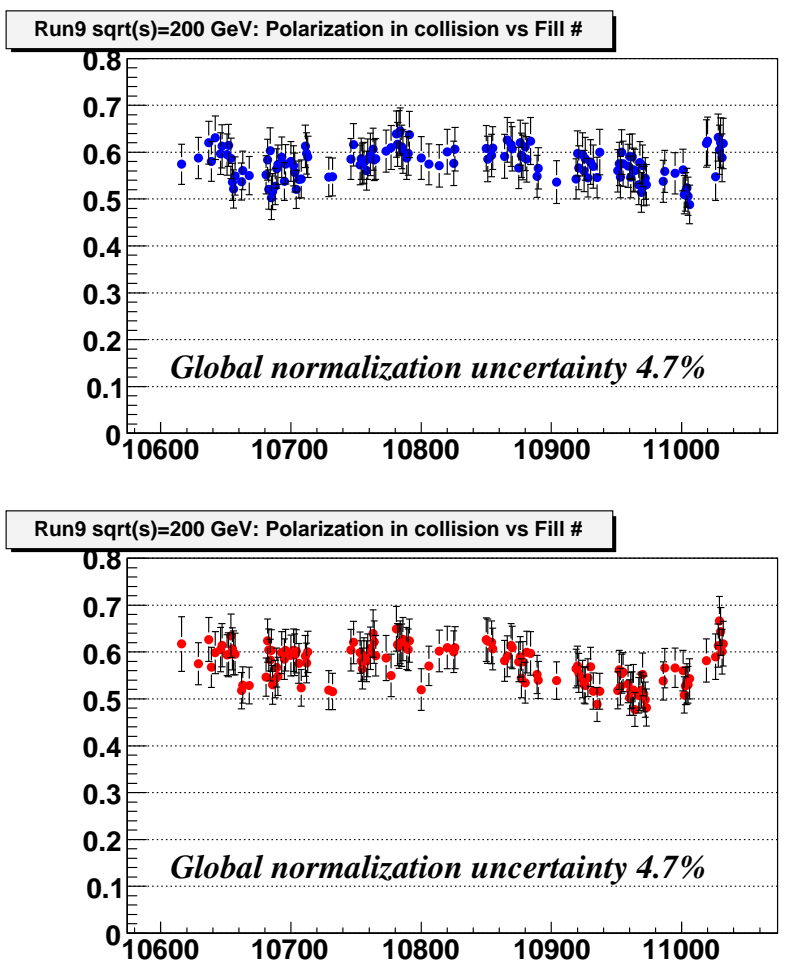

Figure 54. The same as Fig. 53, but for $\sqrt{s}=200 \mathrm{GeV}$ measurements. 
Table 5

Global systematic uncertainties (fill-by-fill correlated). The HJet uncertainties are discussed in [5]; "Pol. profile" relates to the uncertainty in the average pol. profile discussed in Section 6.2 (variations in two trans. directions considered as correlated); "Time dependent inconsistency" derived from the "global" inconsistency between Polarimeter-1 and Polarimeter-2 measurements in Fig. 26 and 27.

\begin{tabular}{|l|c|c|c|c|}
\hline \hline & $\begin{array}{c}\text { Blue } \\
500 \mathrm{GeV}\end{array}$ & $\begin{array}{c}\text { Yellow } \\
500 \mathrm{GeV}\end{array}$ & $\begin{array}{c}\text { Blue } \\
200 \mathrm{GeV}\end{array}$ & $\begin{array}{c}\text { Yellow } \\
200 \mathrm{GeV}\end{array}$ \\
\hline HJet normalization, stat. & $2.5 \%$ & $2.7 \%$ & $1.0 \%$ & $1.0 \%$ \\
HJet normalization, syst. (dilution) & $2 \%$ & $2 \%$ & $2 \%$ & $2 \%$ \\
HJet normalization, syst. (backgr) & $3 \%$ & $3 \%$ & $2 \%$ & $2 \%$ \\
Time dependent inconsistency & $5 \%$ & $10 \%$ & $3 \%$ & $3 \%$ \\
Pol. profile & $5 \%$ & $5 \%$ & $2 \%$ & $2 \%$ \\
\hline Total & $8.3 \%$ & $12.1 \%$ & $4.7 \%$ & $4.7 \%$ \\
\hline \hline
\end{tabular}

two polarized beams are combined) the relative uncertainty is $4.4 \%$ for $\sqrt{s}=200 \mathrm{GeV}$ data and $9.2 \%$ for $\sqrt{s}=500 \mathrm{GeV}$ data.

For two physics fills, 10528 (blue and yellow) and 11020 (yellow), pC polarimeter failed to provide reliable measurements, so for the final polarization values for these fills we used HJet measurements, corrected for the average polarization profile for the corresponding beam energy; for the syst. uncertainty we used only "Pol. profile" uncertainty from Table 4. Also to mention, fill 10464-blue showed a sharp drop in polarization to zero during the store, so we don't recommend to use it in spin asymmetry analysis (and we don't provide polarization result for it).

\section{Summary}

See abstract.

\section{A. Systematic effects studies}

\section{A.1. Rate issues}

In some parts of Run9 generator pulses with a fixed frequency and amplitude injected in the system on the preamp level were used to study and monitor rate effects (usually in bunch 0). The amplitude and ToF (relative to bunch 0) of these pulses were set up in such a way that they do not interfere with $\mathrm{C}$ events (banana area) - near $1 \mathrm{MeV}$ in energy equivalent and $70 \mathrm{~ns}$ of ToF, as it is shown for example in Fig. 55-top-left. The gen. pulse amplitude in a strip was distributed roughly with $\sigma=2-3$ counts, with a larger variation from strip to strip (e.g. in Blue1 in Fig. 55 with $\sigma \sim 8$ counts), while the ToF variation was small. As it is seen from the comparison of Fig. 55 and 56, in high rate condition we obviously start experiencing problems in measuring both the event rate (miscounting it) and the amplitude (gain supressed). The ToF doesn't show any obvious signs of distortion. Fig. 57 demonstrates how the gen. pulse amplitude distribution is 

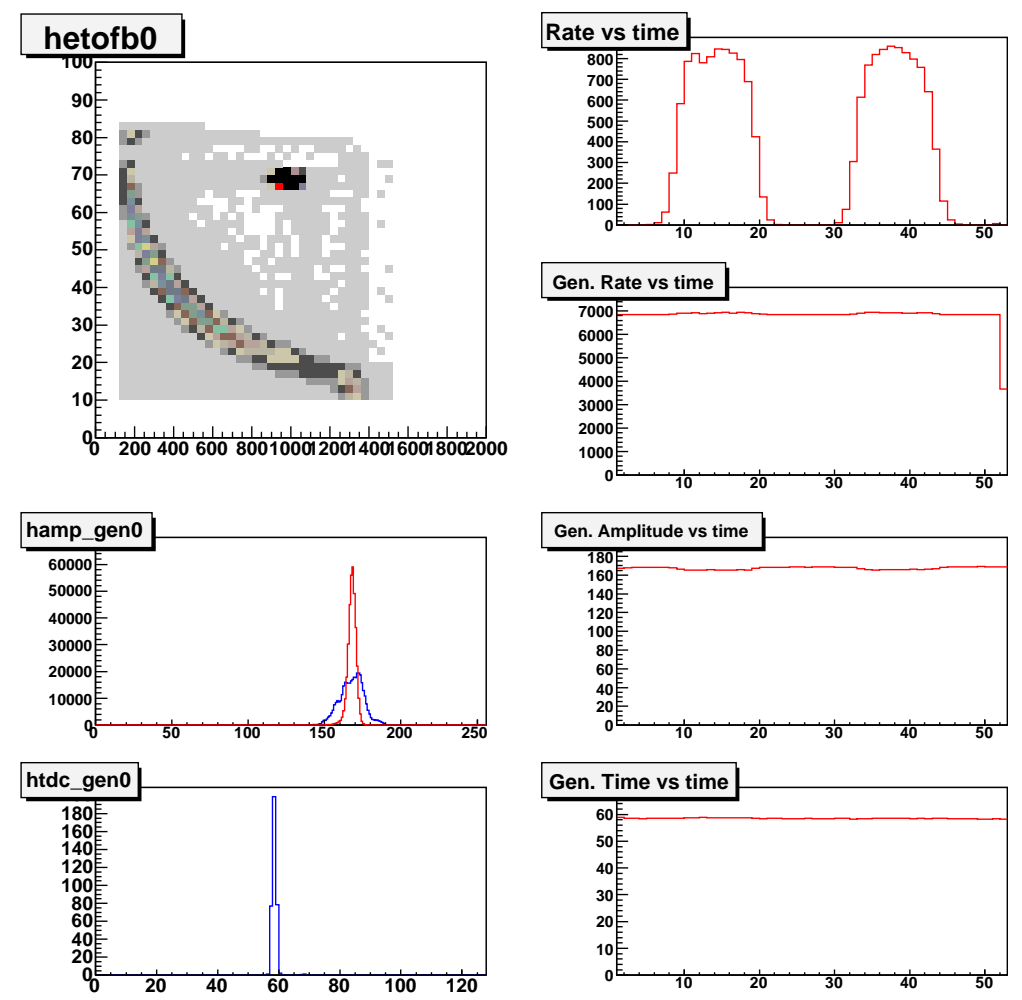

Figure 55. Measurement 10429.013 (Blue1, low rate: $20 \mathrm{kHz} /$ strip), all strips combined. Left-top: ToF (ns) vs deposited energy (keV); "banana" area corresponds to recoil C, activity near ( $1 \mathrm{MeV}, 70 \mathrm{~ns})$ corresponds to injected generator pulses; Left-middle: blue - amplitude distribution (in counts), red - aligned amplitude distribution (peak values of amplitude distributions from gen. pulses aligned in all strips); for the area ToF $>60 \mathrm{~ns}$, ampitude $>400 \mathrm{keV}$. Left-bottom: ToF distribution (in counts); for the area ToF $>60 \mathrm{ns,}$ ampitude $>400 \mathrm{keV}$. Right column plots show time dependence in 1 sec. bins: event rate in the banana, gen. pulse rate, gen. pulse amplitude and gen. pulse time of flight.

distorted when the target reaches the beam center (maximum carbon event rate).

High rate problems were first detected in previous years, when we accidently put a thick target and observed a bunch dependent asymmetry - similar to what is shown in Fig. 59 and 60 (compare to Fig. 58 with no such effect). Further tests showed that the difference between up and down bunch asymmetries (distance between red and blue points in Fig. 59 and 60) on the average was not bunch dependent (within stat. uncertainties), which means that the detector left-right asymmetry gradually changes with bunch number after the abort gap.

Similar bunch dependence was observed in the reconstructed recoil carbon mass in high rate conditions (compare Fig. 61 and 62), which can come from the bunch dependence of either amplitude or time of flight measurements in our system; it can be slightly different in the left-right (or up-down) detectors, and so introducing a detector left-right (or updown) asymmetry. 

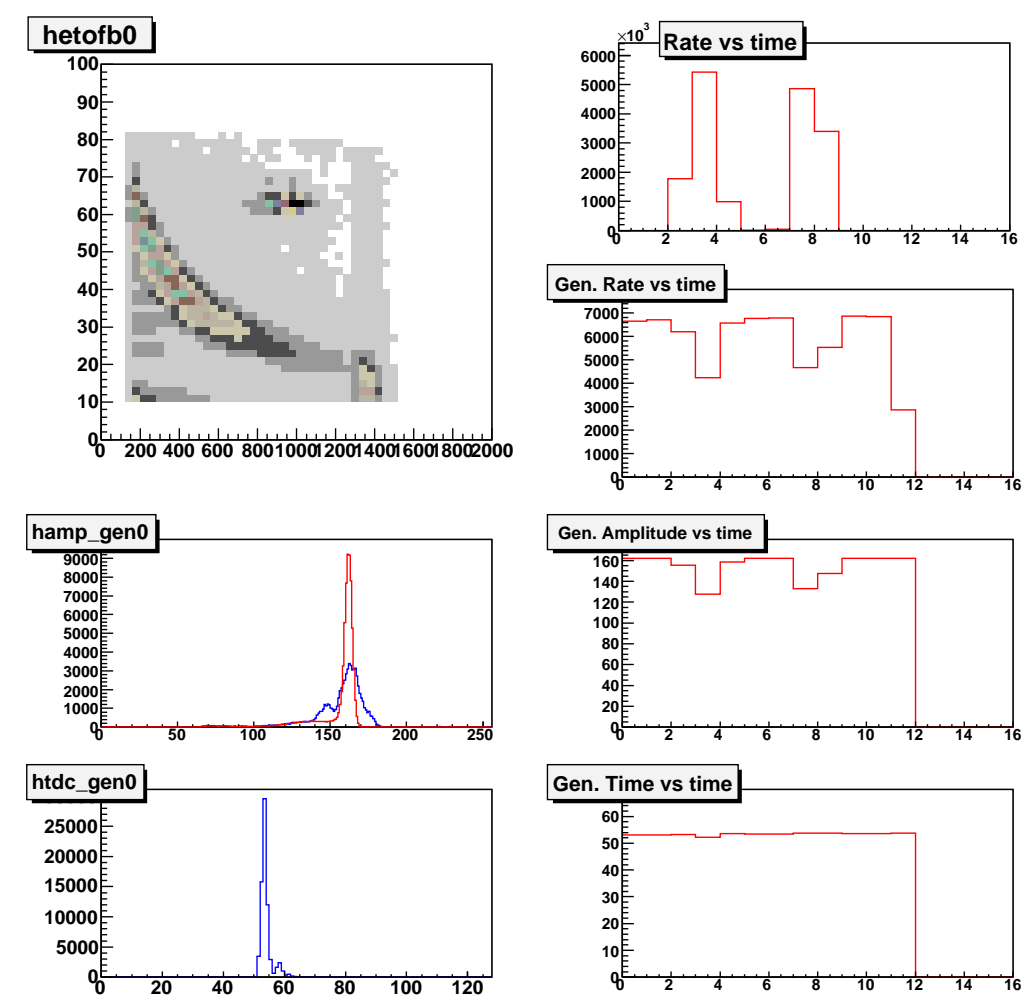

Figure 56. The same as Fig. 55 but for the measurement 10450.116 (Yellow1, high rate: $110 \mathrm{kHz} / \mathrm{strip})$.

\section{A.2. Hamamatsu detectors vs BNL detectors}

Below we compare the bahavior of the Hamamatsu strip and BNL strip detectors installed in the Blue2 polarimeter (detectors 1 and 4 and detectors 0, 2, 3 and 5, correspondingly). We already compared the time dependence of the detector response to alpha particles (Fig. 8) and the fill (time) dependence of "Dead layer" and T0 parameters (Fig. 21 and 18). Despite that the amplitude characteristics of two types of detectors are obviously different (the average response to alphas and "Dead Layer"), the time (fill) dependence was roughly similar, except for the energy slope parameter shown in Fig. 63 and 64. An example of the energy distributions with different slopes in the Hamamatsu detector is shown in Fig. 65. This effect may also be correlated to the instability of the ratio of the beam spin asymmetry vs fill measurements using 45 degree (BNL) detectors and 90 degree (Hamamatsu) detectors at $\sqrt{s}=200 \mathrm{GeV}$, see Fig. 67 for Blue2. The average ratio for the fills $10840-10900$ is $0.648 \pm 0.011$, while for the fills $10920-11030$ the ratio is $0.731 \pm 0.07$. At the same time the instability of the energy slope parameter didn't appear in polarizations for $\sqrt{s}=500 \mathrm{GeV}$ measurements beyond stat. uncertainties (Fig. 66). Notice that the expected ratio here is $\sim 0.707(=\sqrt{2} / 2)$. Slight discrepancy between this value and the observed ratio of $\sim 0.68$ may come from a slight geometrical misalignment of the 45 degree detectors and masked strips. 

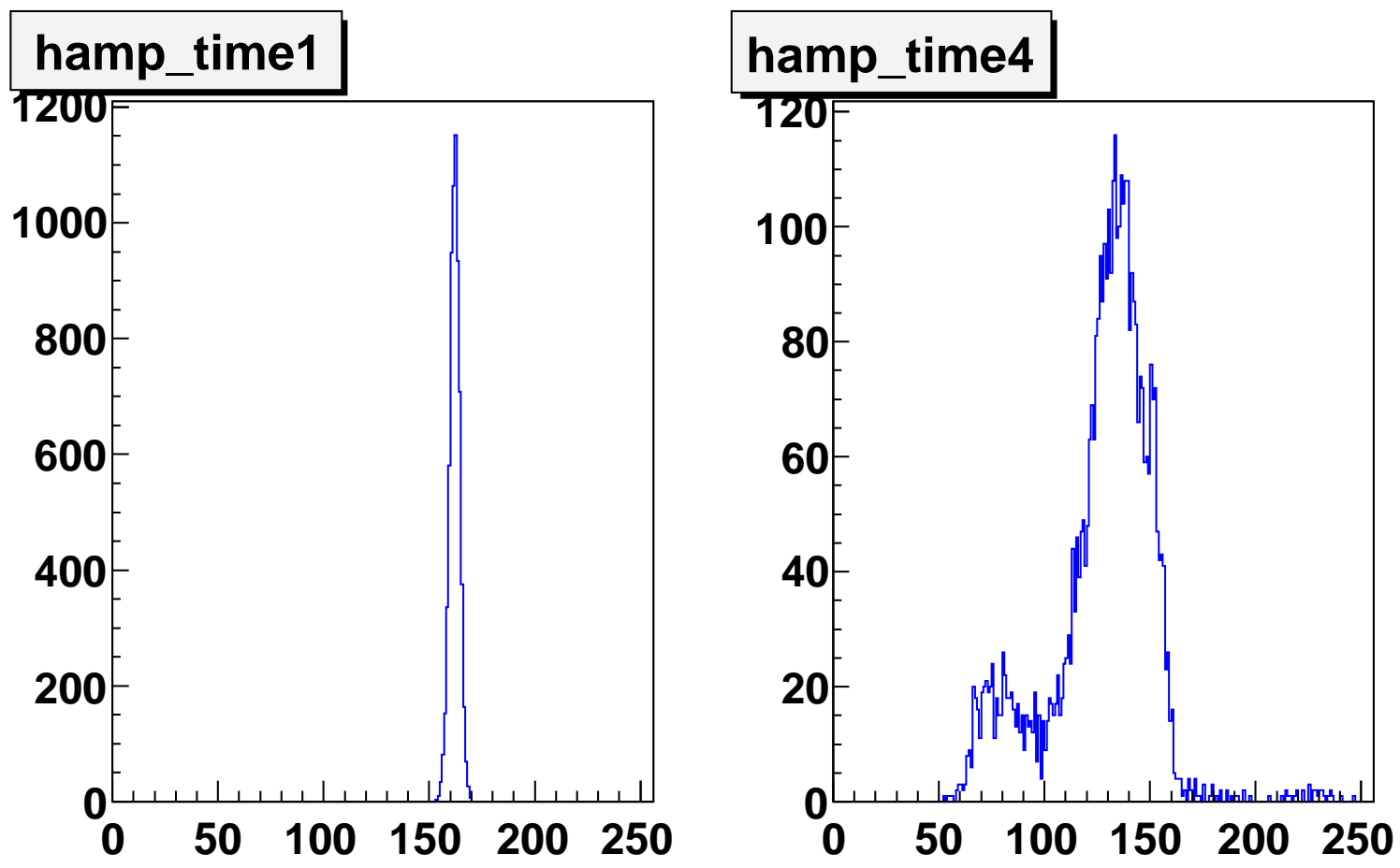

Figure 57. From the measurement in Fig. 56. Distribution of the gen. pulse amplitude (all strips combined). Left: target is out of the beam (time bin 0-1 sec. in Fig. 56); Right: target at the beam center (time bin 3-4 sec. in Fig. 56). 


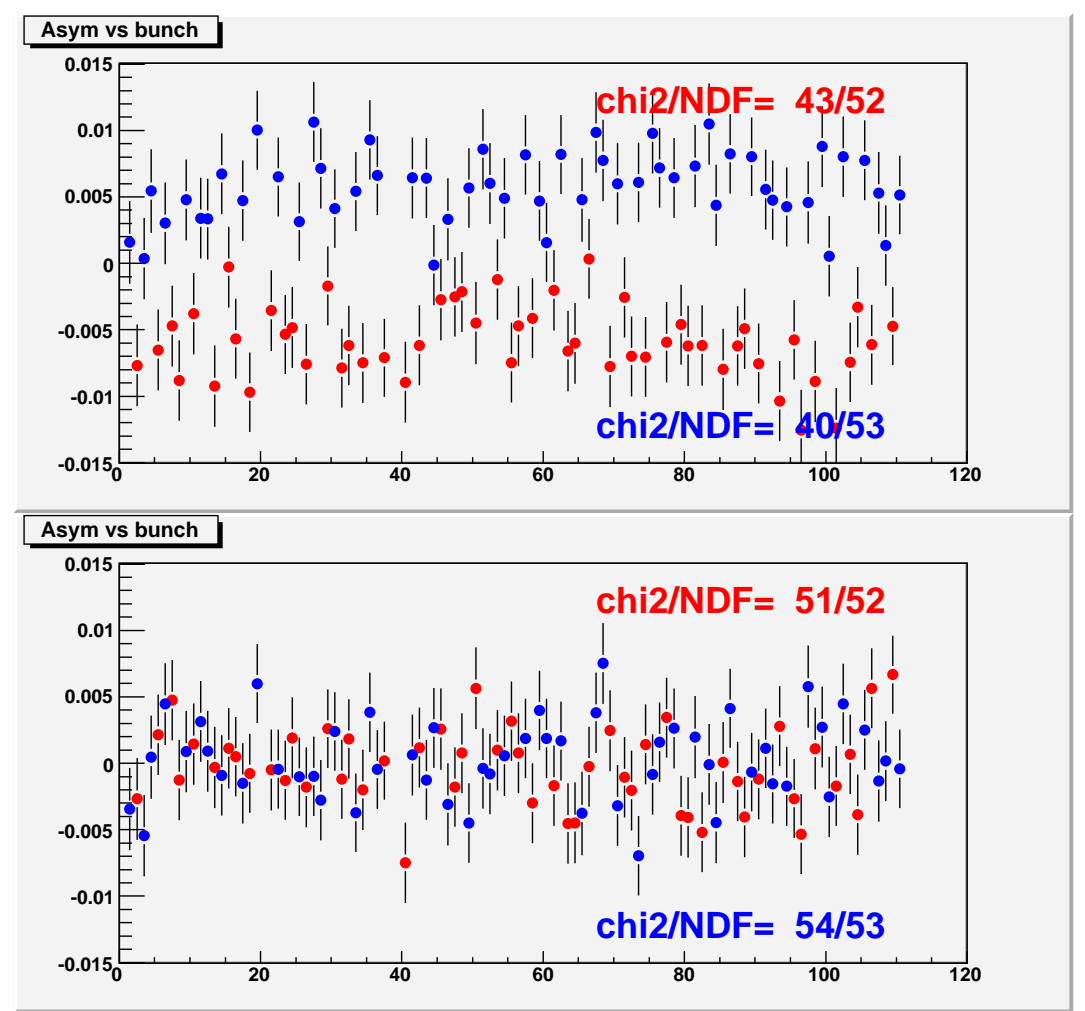

Figure 58. Measurement 10490.005 (Blue1, reasonble rate: $60 \mathrm{kHz} / \mathrm{strip}$ ): bunch by bunch asymmetry corrected for the average over bunches detector asymmetry; blue points for bunches with spin up, red points for bunches with spin down; top plots for leftright asymmetry, bottom plot for up-down asymmetry (supposed to be zero for vertically polarized beam). 

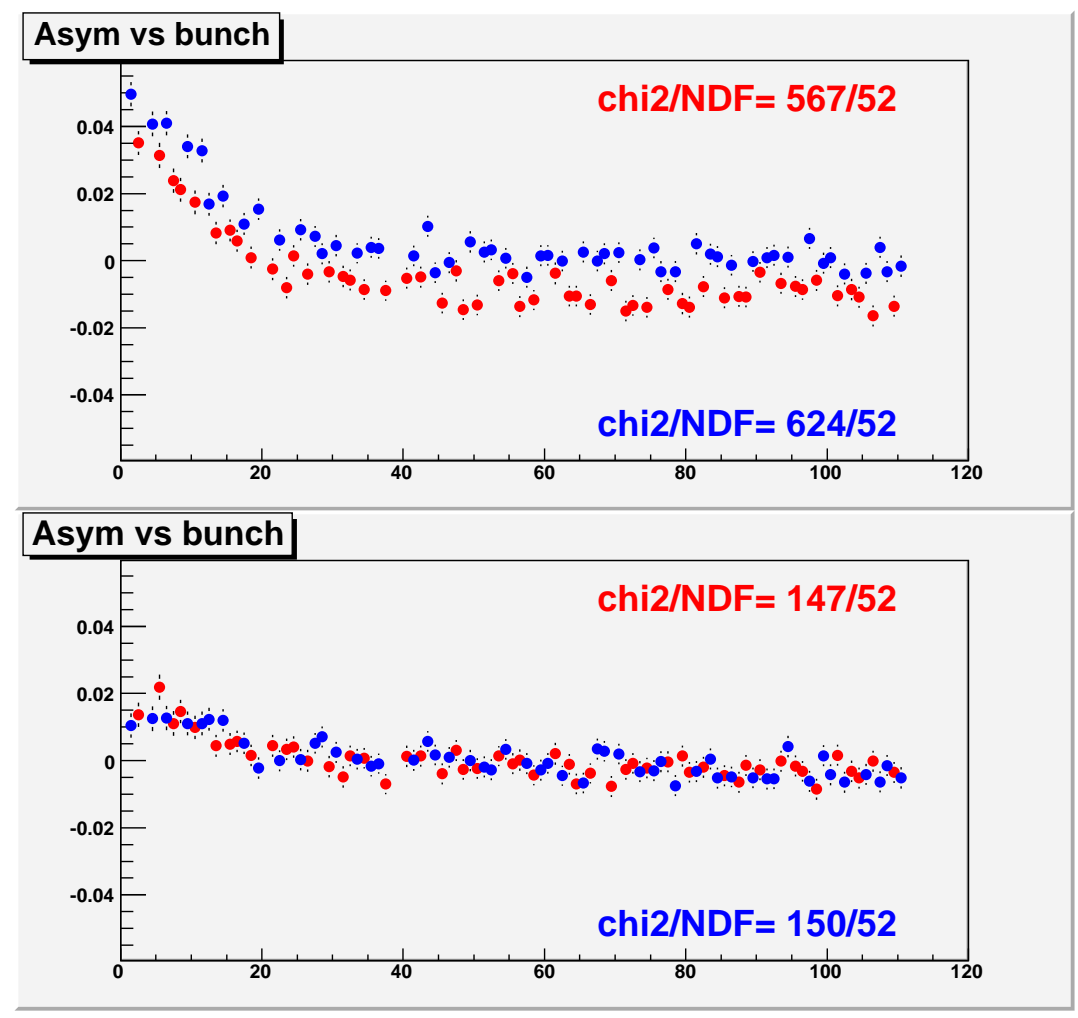

Figure 59. The same as Fig. 58 but for the measurement 10439.008 (Blue1, high rate: $120 \mathrm{kHz} / \mathrm{strip})$.
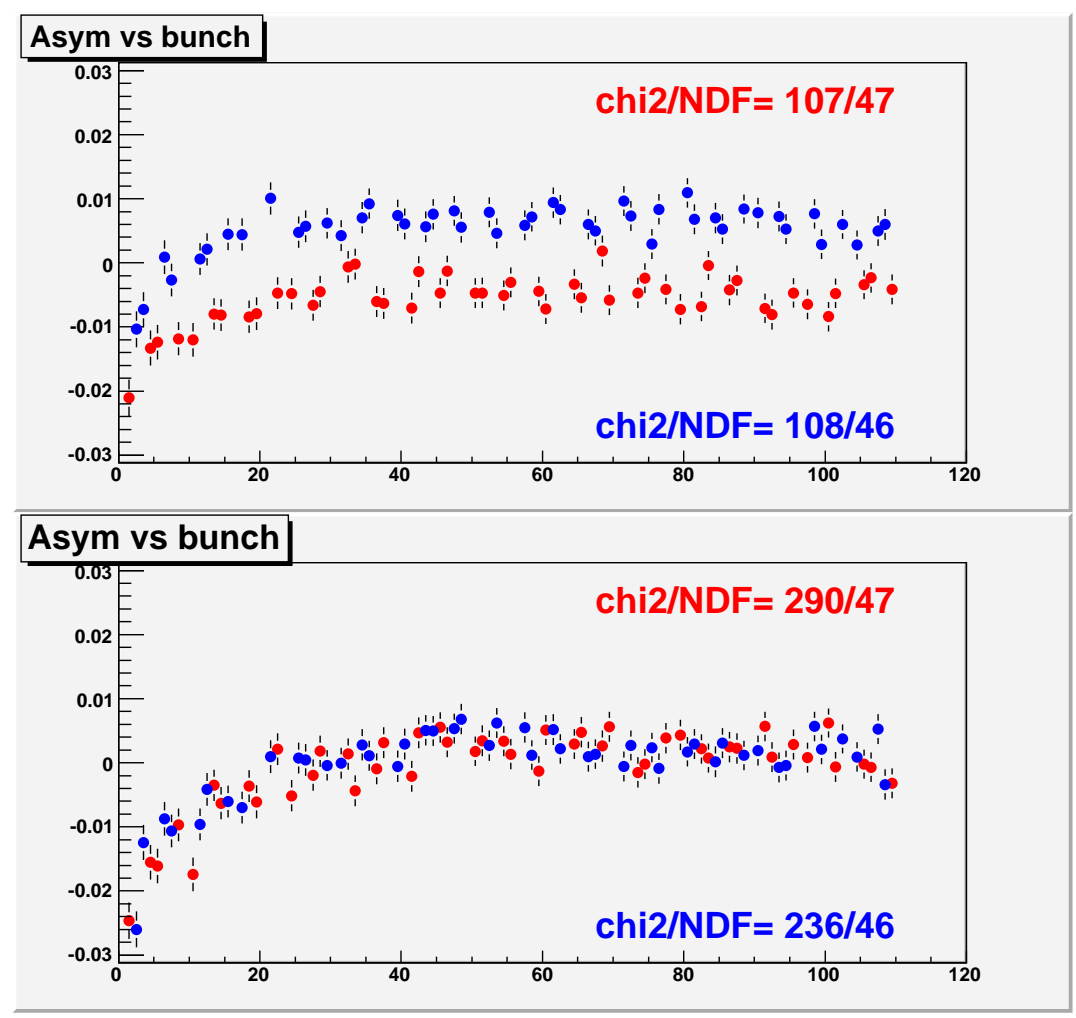

Figure 60. The same as Fig. 58 but for measurement 10402.310 (Yellow2, high rate). 

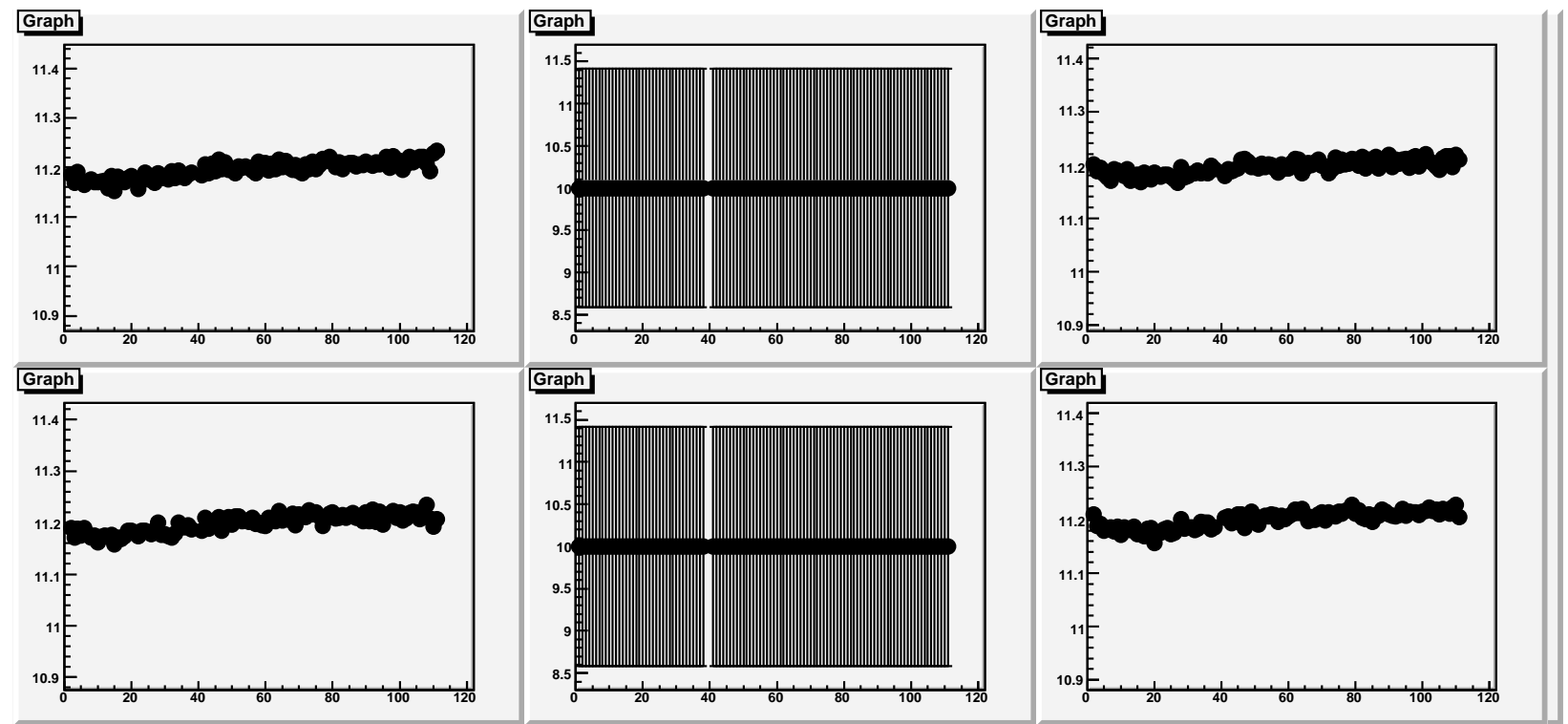

Figure 61. Measurement 10490.005 (Blue1, reasonble rate: $60 \mathrm{kHz} /$ strip, Reconstructed carbon mass $\left(\mathrm{GeV} / \mathrm{c}^{2}\right)$ from the measured recoil carbon energy and time of flight, in detectors 0,1 and 2 (upper row) and 3, 4 and 5 (lower row); detectors 1 and 2 (at 90 degree) were off due to horizontal target used for this measurement.
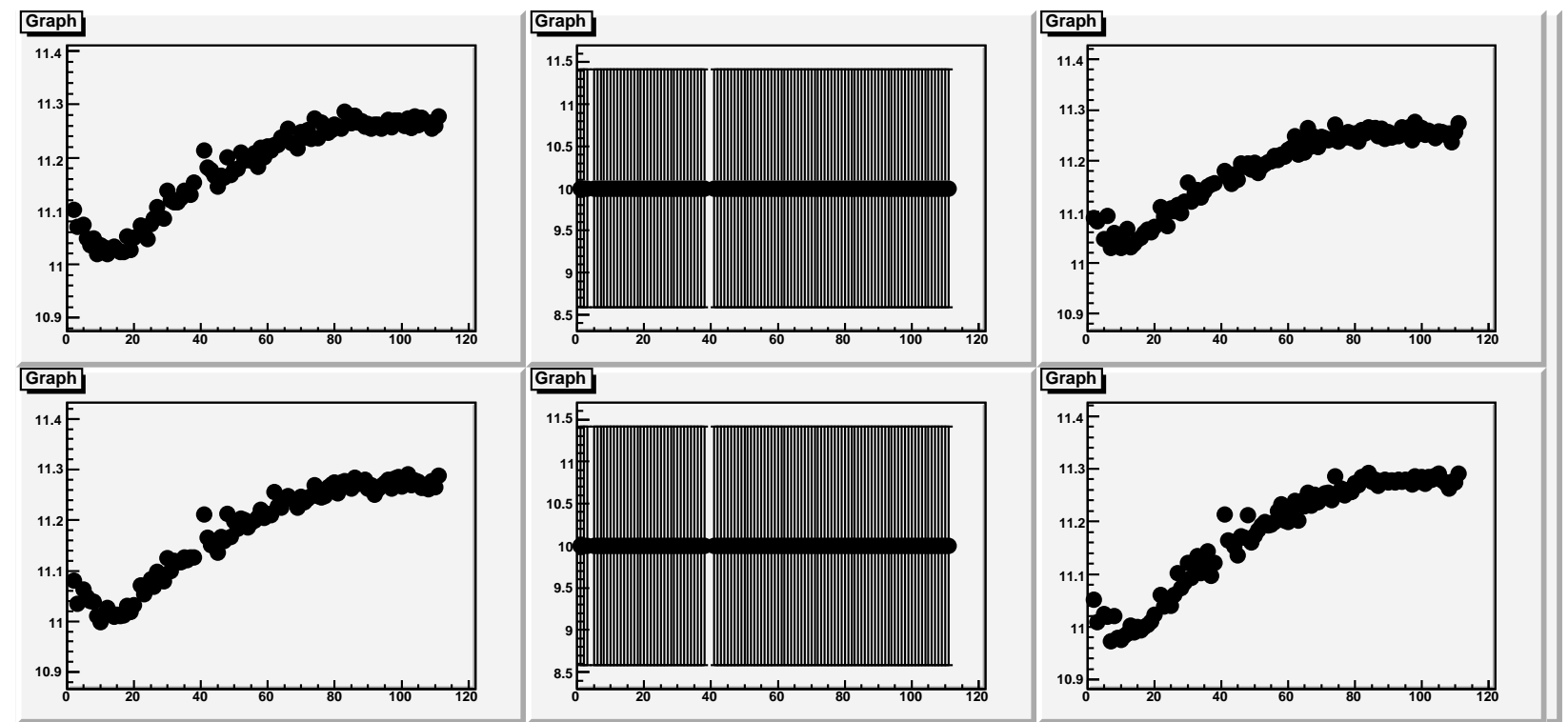

Figure 62. The same as Fig. 61 but for the measurement 10439.008 (Blue1, high rate: $120 \mathrm{kHz} /$ strip). 


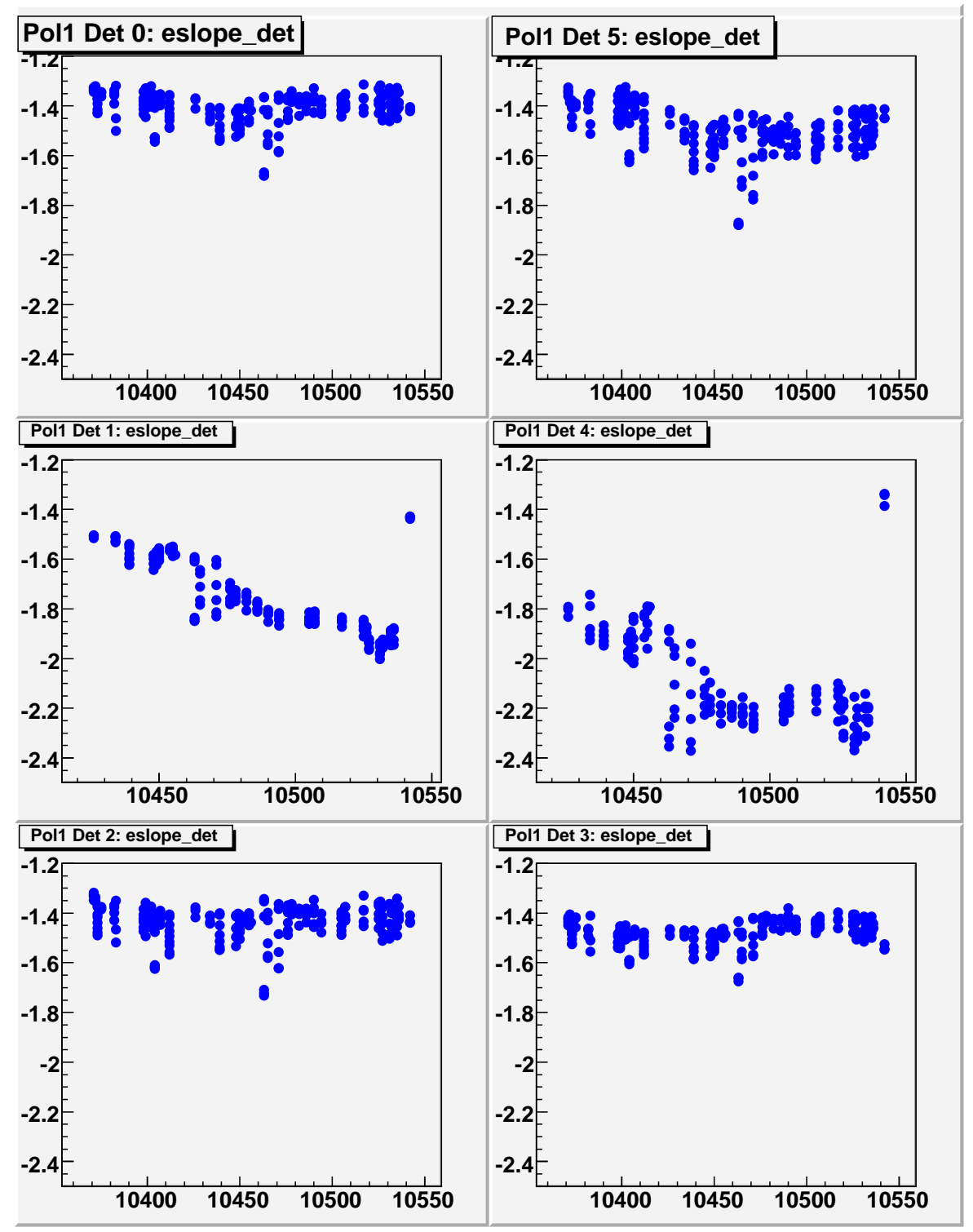

Figure 63. The average over the detector strips the slope of the fit of $d N / d E_{k i n}$ vs $E_{k i n}$ to exponent times $10^{-3}$ in $\sqrt{s}=500 \mathrm{GeV}$ measurements by Blue 2 vs fill number (each fill usually had several measurements, all of them are shown here); all 6 detectors are shown, detectors 1 and 4 (middle plots) being Hamamatsu strip photodiods, all others being BNL's Si detectors; only measurements with vertical target are shown. 

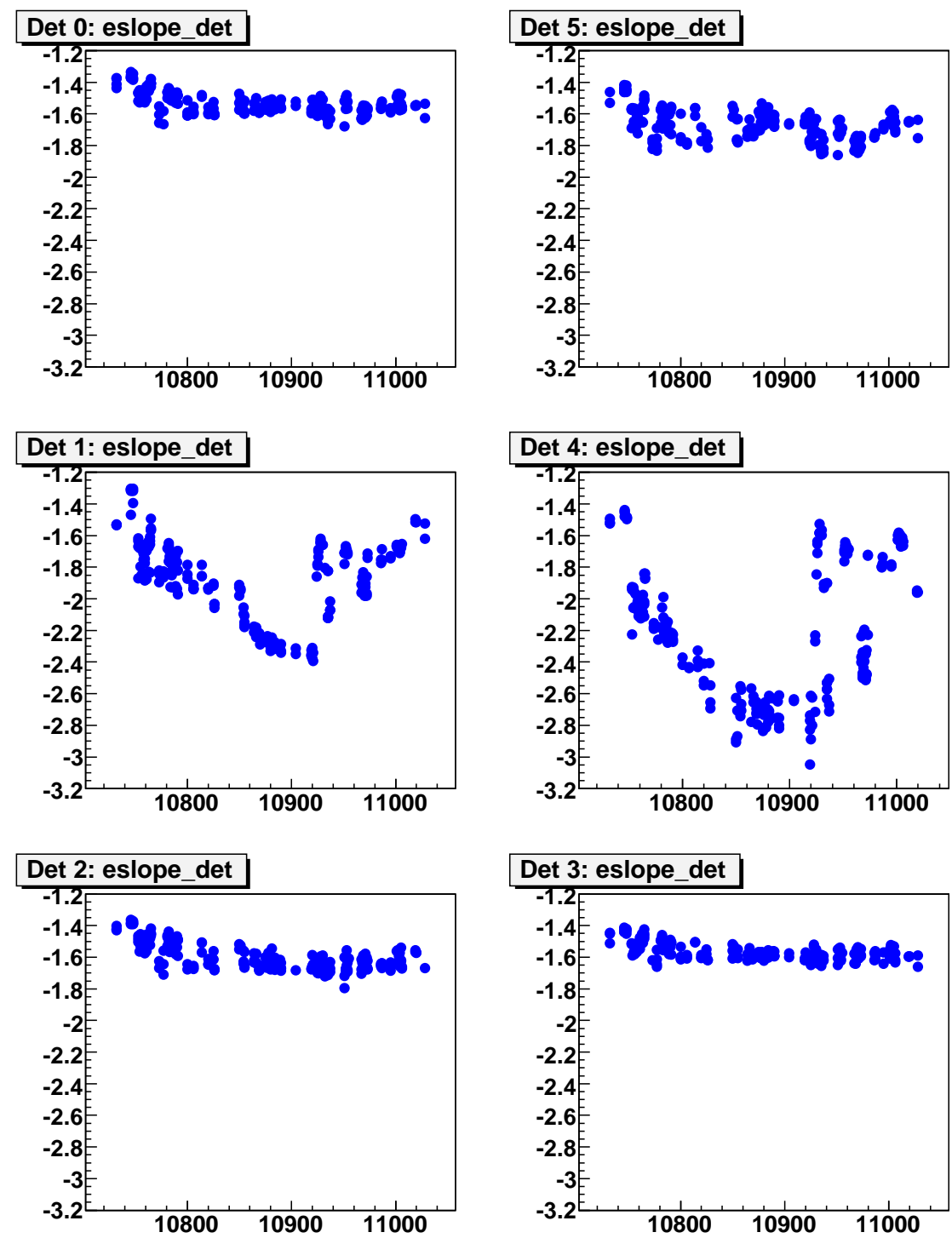

Figure 64. The same as Fig. 63, but for $\sqrt{s}=200 \mathrm{GeV}$ measurements. 


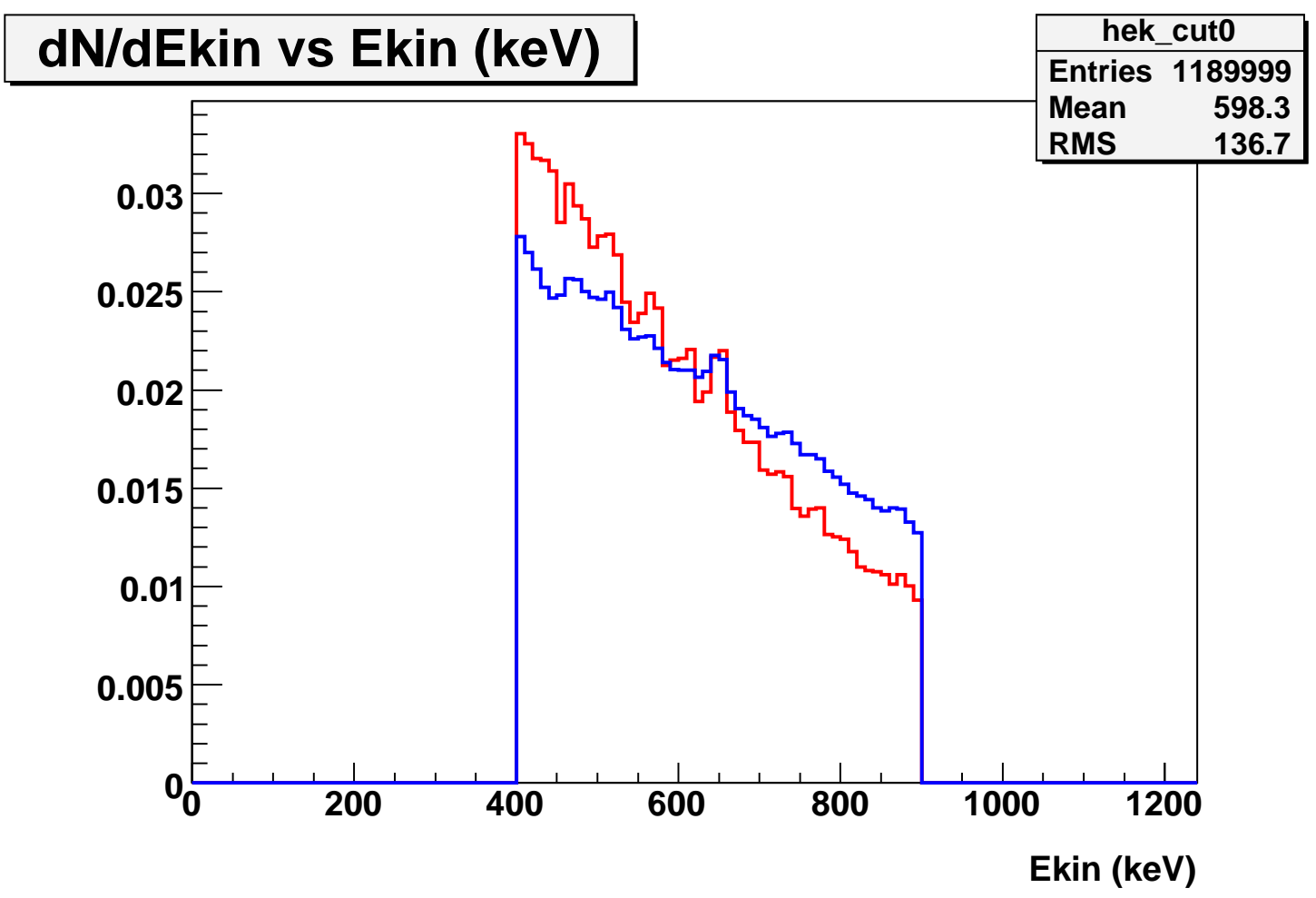

Figure 65. The average normalized recoil Carbon kinetic energy distributions $\left(d N / d E_{k i n}\right.$ vs $E_{k i n}$ ) for the $E_{k i n}$ range $400-900 \mathrm{keV}$ for the strips in detector 4 (Hamamatsu strip) of Blue2, red for measurement 10746.204, blue for 10854.204; the slope parameters of exp fit are $-1.5 \cdot 10^{-3}$ and $-2.5 \cdot 10^{-3}$ for these two measurements, respectively. 


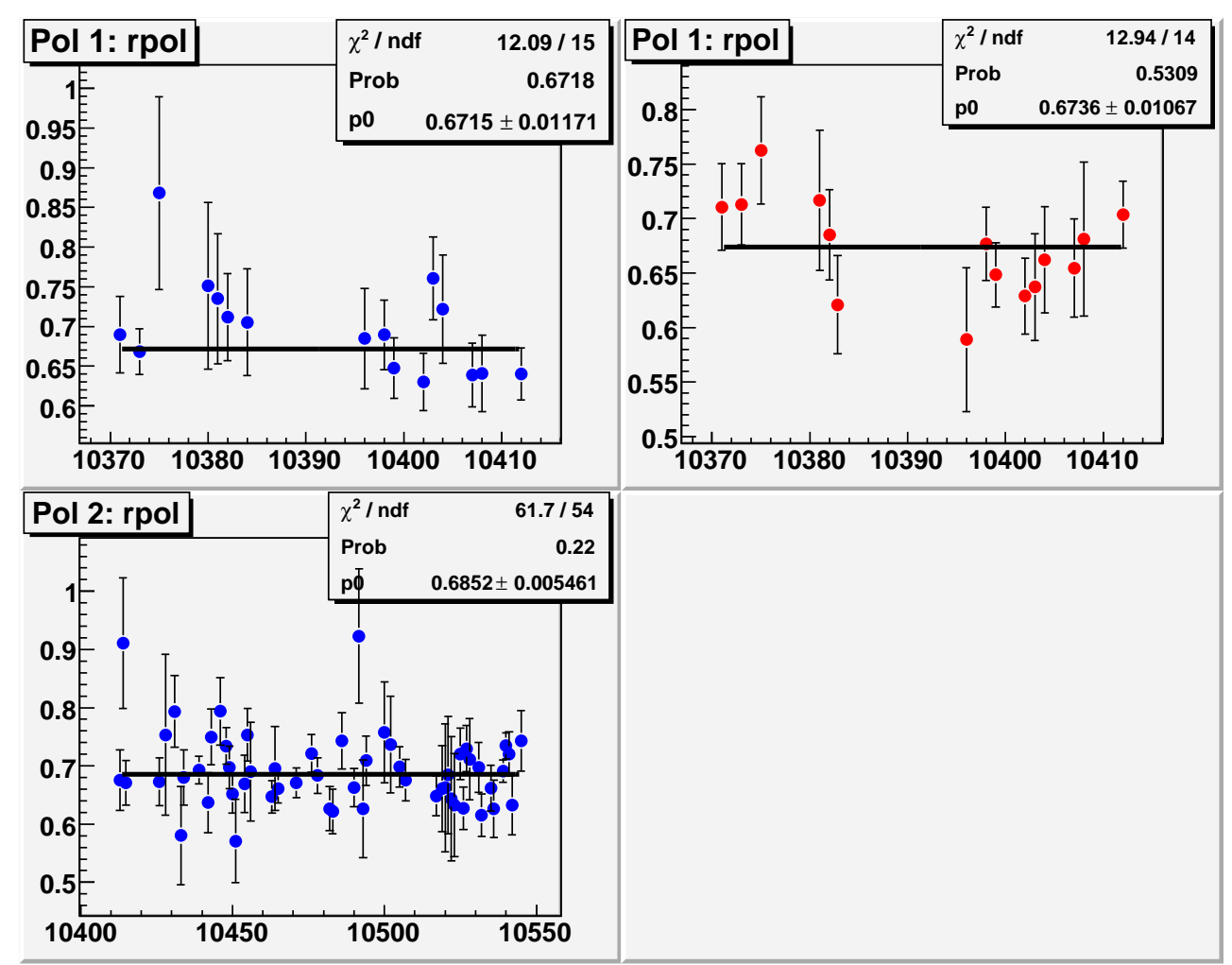

Figure 66. The fill dependence of the ratio of the beam spin asymmetries measured by 45 degree (BNL-type) detectors and 90 degree (Hamamatsu-type in Blue2 and BNL-type in Blue1 and Yellow1) detectors; top-left - Blue1, bottom-left - Blue2 and top-right - Yellow1 (in Yellow2 45 degree detectors were not used. 

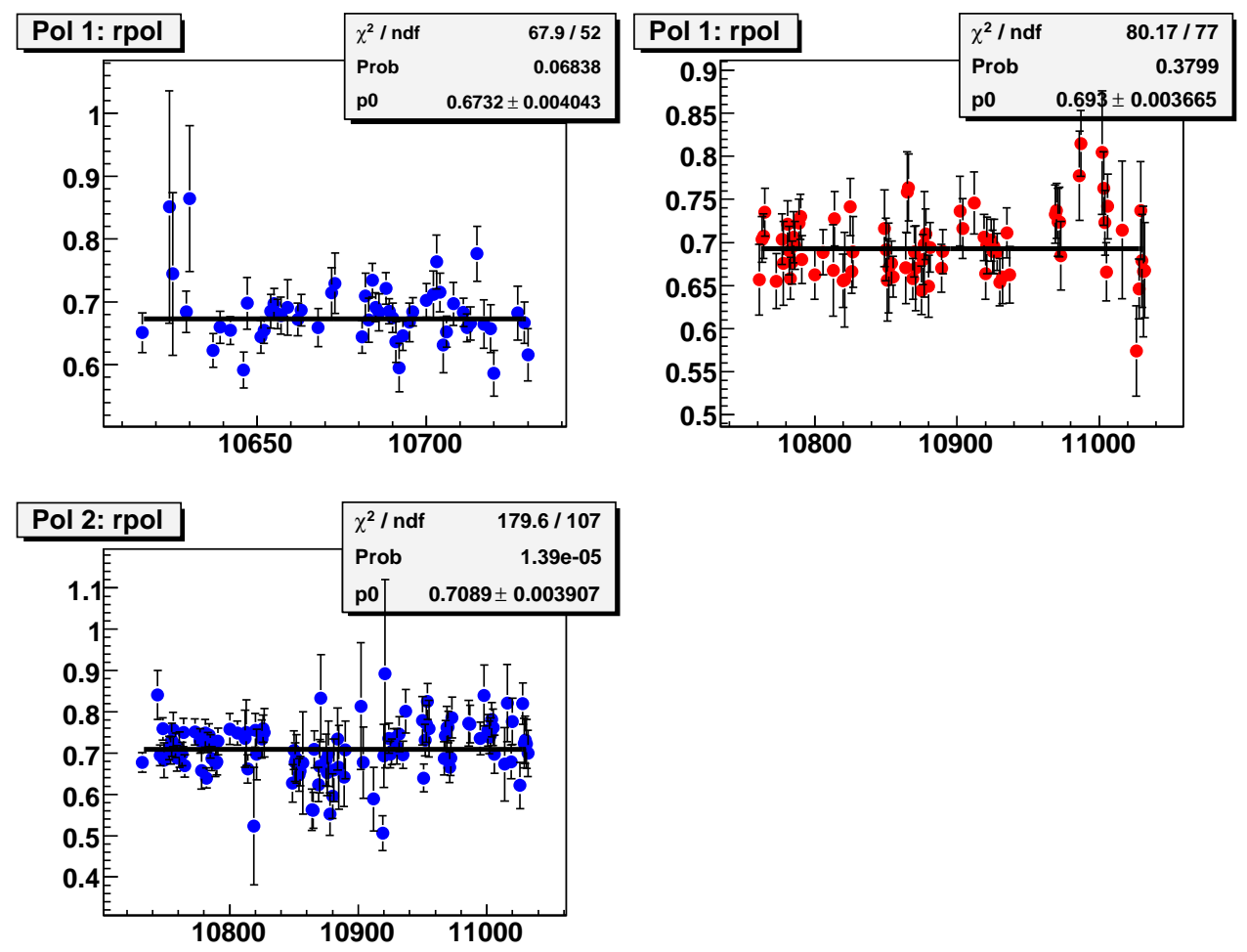

Figure 67. The same as Fig. 66 , but for $\sqrt{s}=200 \mathrm{GeV}$ measurements; notice that Blue1 didn't have measurements for fills $>10773$.

\section{REFERENCES}

1. I. Nakagawa, at al, RHIC/CAD Accelerator Physics Note 275, 2007.

2. V. Dharmawardane, at al, RHIC/CAD Accelerator Physics Note 355, 2009.

3. I. Nakagawa, private comminication.

4. Haixin, talk at RSC meeting Oct 2, 2009.

5. S. Lee et al, AN on HJet Run9 analysis, to be submitted. 